\title{
Improved Description of Hafnium Spectra
}

\author{
Charles H. Corliss and William F. Meggers
}

\begin{abstract}
Earlier descriptions of atomic hafnium spectra, from conventional ares or sparks, have been handicapped by the absence of pure samples and by the presence of a strong background of molecular spectra. Recent availability of highly purified hafnium metal, and the development of a new light source, removed these handicaps. This improved description of hafnium spectra was made by employing electrodeless metal-halide lamps excited, at relatively low pressure and temperature, by microwaves. Lamps of hafnium iodide, hafnium bromide, and hafnium chloride were compared to recognize the spectra of halogens or their compounds and thus arrive at the atomic hafnium lines common to different lamps. The number of lines now ascribed to hafnium spectra exceeds 6,200 as compared with about 2,400 heretofore. The wavelengths range from 1284.88 to $12043.08 \mathrm{~A}$, and estimated relative intensities in different light sources (including ares, sparks, and electrodeless lamps) indicate which lines belong to Hfr, HfII, HfIII, and Hf IV. The splitting of spectral lines in magnetic fields (Zeeman effect) has also been improved by using magnetic fields of higher intensity, and greater spectrographic resolving power than before. The number of lines for which Zeeman patterns have been observed has been increased from 280 to 1,030 , and the types of complex patterns invariably confirm the assignments of lines to HFI or HfII.
\end{abstract}

Hafnium was the next to the last stable element to be discovered in the earth's crust; its discovery was announced by Coster and Von Hevesy [1] ${ }^{1}$ in a letter to Nature on January 20, 1923 . That year Hansen and Werner [2] published the first description of the optical spectra of hafnium, consisting of wavelengths of 807 lines ranging from 2253.98 to $7240.9 \mathrm{~A}$, and estimated relative intensities in arc and spark spectra.

In 1925 Professors Bohr and von Hevesy generously presented about 0.2 gram of their purest hafnium to Meggers [3] who made another description of hafnium arc and spark spectra for the purpose of finding regularities among the lines. Spectrograms were made by burning small portions of the sample in a silver arc or spark, and wavelengths from 2155.72 to $9250.27 \mathrm{~A}$ were measured for some 2,100 spectral lines. However, careful comparison with known spectra proved that 609 of the observed lines were due to impurities, among which niobium, zirconium, and titanium were the most abundant. Nevertheless this list of about 1,500 hafnium lines, because of its effective separation of $\mathrm{Hf}$ I and Hf II spectra by comparing arc and spark intensities, permitted the detection of regularities in both spectra.

In 1928 Meggers and Scribner reported [4] the first multiplets in Hf II, and in 1930 they published [5] the first regularities in $\mathrm{Hf} \mathrm{I}$. It was recognized that extension and confirmation of these first regularities would require further improvements in the basic descriptions of hafnium spectra and also observation of the Zeeman effect. Both requirements were partially met in 1932 when hafnium spectra were reobserved with purified oxide donated by Prof. von Hevesy, and the first Zeeman-effect spectrograms were made with a small rod of hafnium metal presented for this purpose by Dr. G. Holst of Eindhoven. The arc and spark spectra were remeasured, and extended from 1990.73 to $10637.92 \mathrm{~A}$. The new sample of hafnium oxide was entirely free from

1 Figures in brackets indicate the literature referances on page 272 . titanium and showed only a trace of niobium, but it contained considerable amounts of zirconium and nickel. The number of spectral lines positively identified with hafnium atoms or ions was increased from about 1,500 to 2,400 , including nearly 1,400 Hf I, about 1,000 Hf II, and several dozen Hf III lines. Magnetic splitting (Zeeman effect) was observed for 280 hafnium lines (2393 to 4817 A), 70 belonging to the first and 210 to the second spectrum. These confirmed the first reported $[4,5]$ regularities in the spectra, and full details of the new wavelengths, intensities, Zeeman data, and quantum interpretation of the second spectrum of hafnium were published by Meggers and Scribner [6] who promised that "Further data and analysis of the Hf I spectrum will be published after they have been supplemented by additional observations of Zeeman effects, since the data at present available have proved insufficient for the complete identification of Hf I spectral terms"

Further progress in the investigation and interpretation of the Hf I spectrum was delayed many years for the following reasons: it was proved that the Zeeman data were inadequate, many impurity lines were always present, and it was suspected that the line list was far from complete. In his 1928 paper on wavelength measurements in spectra of hafnium Meggers stated [2] "The greatest difficulty in describing the arc spectrum of hafnium arises from the band spectrum which probably originates with the oxides. Some of these bands are very strong and extremely complex over long spectral ranges. In many cases it is impossible to decide if faint lines belong to $\mathrm{Hf}$ I or to the $\mathrm{HfO}_{2}$ spectrum". Attempts to improve the description of the hafnium are spectrum with electrodes of solid hafnium metal kindly donated in 1951 by C. P. Keim of the Oak Ridge National Laboratory were frustrated by the strong background of HfO spectra from 3000 A to the infrared limit of photography, and by the presence of metallic impurities, mostly zirconium.

Before hafnium was discovered, the accepted 


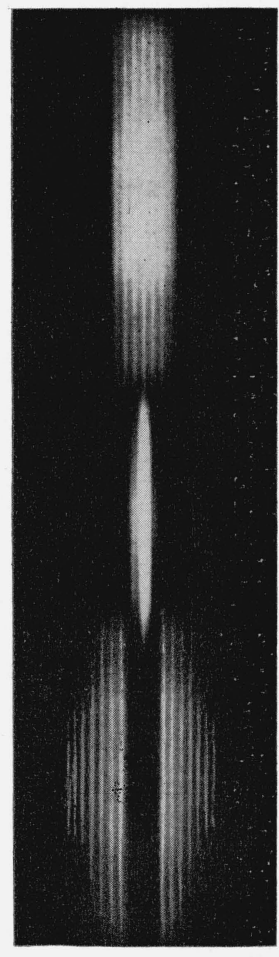

Type I

7740.17

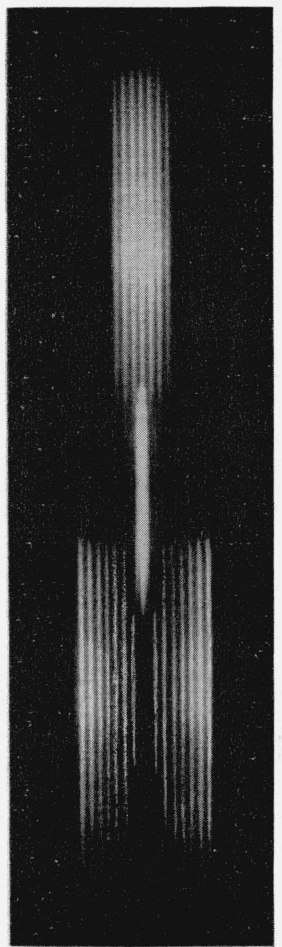

Type 2

6789.27

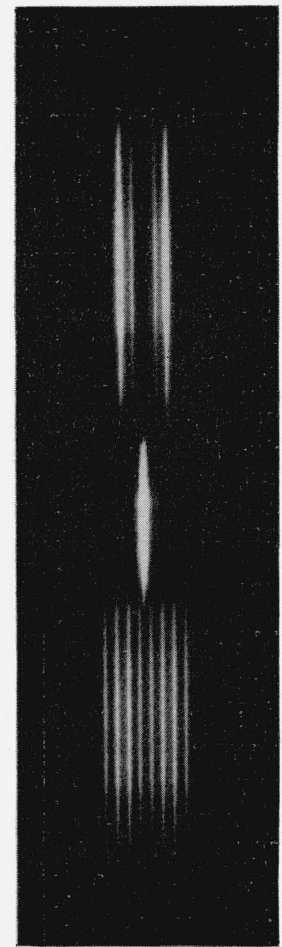

Type 3

6185.13

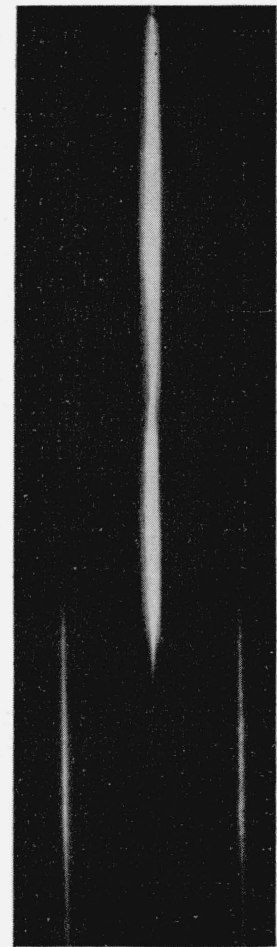

p

no

field

$n$

FIGURE 1. Types of Zeeman patterns for Hf I observed at NBS using electrodeless lamps in a field of 37,000 oersteds.

chemical atomic weight of zirconium [7] was more than a half-unit larger than its present value, 91.22 . This difference was later explained [8] by the unsuspected presence of hafnium as an impurity in the earlier samples of zirconium. Likewise, after information about the spectra of hafnium became available it was found [3] that all earlier descriptions of zirconium spectra contained many hafnium lines. The complete separation of hafnium and zirconium is one of the most difficult chemical operations, and to this day neither has been obtained spectroscopically free from the other. However, since certain properties of these metals have recently been found to be useful in the construction and operation of nuclear reactors, greater efforts have been made to provide large quantities of high-purity hafnium and zirconium.

In December, 1955, two rods of hafnium metal, 1/4-in. diameter, 4-in. length, were generously provided for our further spectroscopic investigations by Stephen M. Shelton of the U. S. Department of Interior, Bureau of Mines. A complete quantitative spectrochemical analysis of these samples was also provided; it expressed the impurities in parts per million as follows: $\mathrm{Cd}<0.5$, B 0.2 , Al 20, Fe 500,
$\mathrm{Cu} 40, \mathrm{~Pb}<10, \mathrm{Cr}<30$, Si $150, \mathrm{Mg} 40, \mathrm{Ti} 100, \mathrm{Zn}$ $<50$, Ni 40, Mn <10, Mo 60, V 40, Co $<5$, $\mathrm{Sn}<5$, Zr 80. Thus the total impurities of these samples approximate 0.1 percent, and zirconium, which was always the most troublesome, constitutes only 0.008 percent. Of these impurities, only the stronger lines of $\mathrm{Fe}, \mathrm{Si}, \mathrm{Ti}, \mathrm{Zr}, \mathrm{Zn}$, and $\mathrm{Al}$ were observed in the electrodeless lamps described below.

Recently, a new type of light source was described by Corliss, Bozman, and Westfall [9], who showed that a bright emission spectrum of any metal could be obtained from out-gassed quartz tubes inclosing a minute amount of any volatile compound of that metal, when excited by microwaves. The first hafnium lamp of this type was made by producing, in an evacuated quartz tube, a chemical reaction between filings of the above-mentioned hafnium metal and a small crystal of iodine. The spectrum emitted by this lamp was photographed from 2000 to $12000 \mathrm{~A}$; it was much richer than any hafnium arc spectrum because it completely suppressed the usual HfO bands that always obscured several thousand hafnium lines of low or moderate intensity. The only defect was the presence of some strong iodine lines and an entirely new system of bands, presum- 


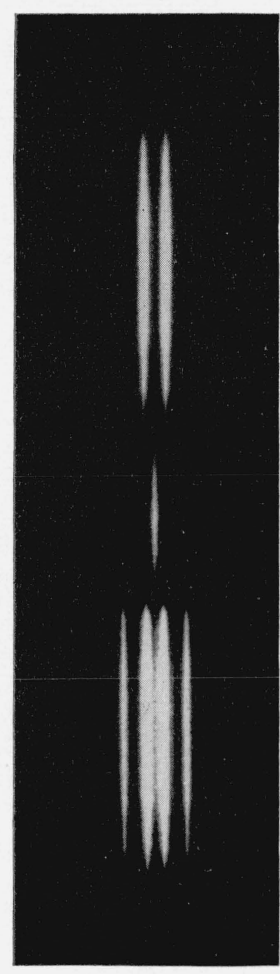

Type 4

5040.82

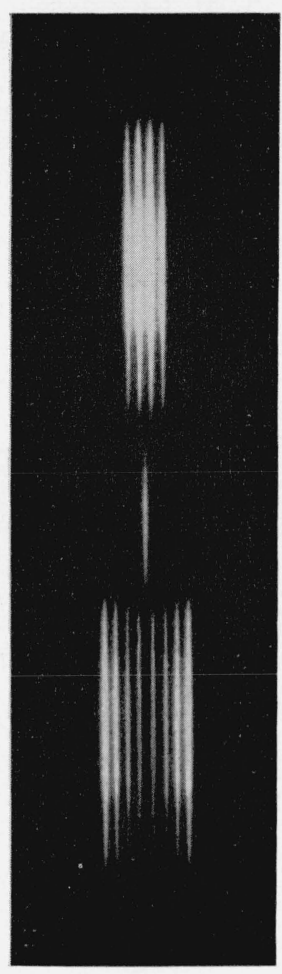

Type 5

6248.95

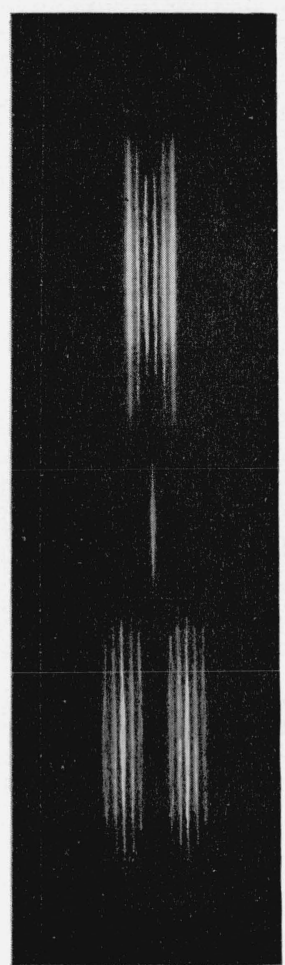

Type 6

6279.84

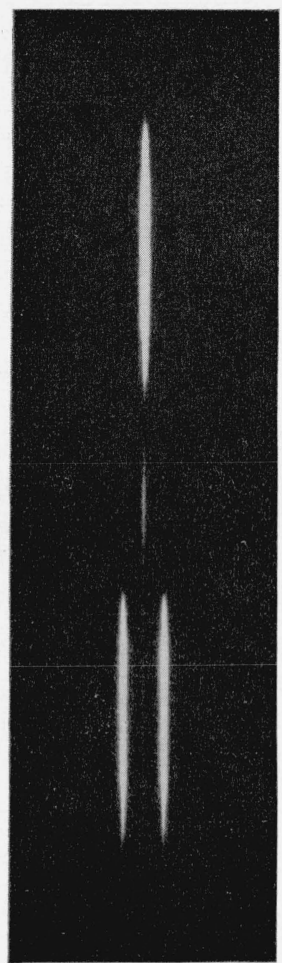

$p$

no field

$n$

FIGURE 2. Types of Zeeman patterns for Hf II observed at NBS using electrodeless lamps in a field of 37,000 oersteds.

ably due to hafnium iodide which appeared with low intensity in several short intervals of spectra. These lines and bands were replaced by others when the lamps were made either with hafnium bromide or with hafnium chloride. Thus by observing and comparing the spectra of two different halides the halogen lines and molecular bands are completely recognized as differences whereas the atomic spectra of hafnium (and its impurities) are mutually confirmed. Such a comparison between hafnium iodide and hafnium bromide spectra was made between 2000 and $6500 \mathrm{~A}$, but, because many very intense bromine lines appear in the red and near infrared, a lamp containing hafnium chloride was used in these regions to confirm hafnium spectra emitted by the hafnium iodide lamp. Among the three hafnium halides, hafnium iodide was the easiest to prepare and its hafnium spectra were more intense and less blended with halogen lines because iodine emits no strong lines between 2062 and $4100 \mathrm{~A}$.

These lamps were excited with a commercial diathermy unit of $125 \mathrm{w}$ power and $2,450 \mathrm{mc}$ frequency, and were observed end on. The spectra above 2000 A were dispersed by $22 \mathrm{ft}$ radius concave diffraction gratings in Wadsworth stigmatic mountings. Two 6-in. gratings were used, one ruled 15,000 and the other 30,000 lines per inch. The spectrographs and photographic plates employed in recording hafnium spectra have been described [10] in a paper on the spectra of rhenium. When the hafnium spectrograms were made no effective way of varying the excitation of the metal halide lamps was available to distinguish $\mathrm{Hf}_{\mathrm{I}}$ from $\mathrm{Hf}$ II lines. Therefore, on each spectrogram a series of exposures was made of successive increments of slit illuminated by the d-c arc in air, the high-voltage condensed spark in air, the spectrum of the hafnium halide lamp, and the arc spectrum of iron. The last provided international standards of wavelength for measurements of hafnium lines by interpolation and the others permitted the assignment of all hafnium lines to the proper atom or ion. It was observed that the hafnium halide lamp emitted Hf I lines with the greatest intensity, sharpness, and number, but it also gave with lower intensity but equal sharpness practically all of the Hf II lines, which usually appear as broad, hazy, or unsymmetrical lines in the spark. Because the metal 
halide lamps operate at a much lower pressure and temperature than the conventional ares and sparks they emit sharper lines, and one should apologize for measuring their wavelengths relative to the international standards obtained from an iron arc at atmospheric pressure.

Below 2000 A a normal incidence vacuum spectrograph containing a $2-\mathrm{m}$ radius glass grating ruled with 30,000 lines per inch was used to photograph hafnium vacuum arc and spark spectra with reciprocal dispersion of $4.2 \mathrm{~A} / \mathrm{mm}$.

In addition to wavelengths and relative intensities of lines in different light sources, it is desirable, for an improved description of hafnium spectra, to observe the Zeeman effect on as many lines as possible. Back and Landé [11] have shown that only 6 types of resolved Zeeman patterns exist, 3 for even multiplicities and 3 for odd. A 7 th type has a single undisplaced $p$ component and two symmetrically displaced $n$ components; it is generally characteristic of singlet terms or other transitions between levels with equal $g$ values. These 7 types have all been observed in hafnium spectra; typical patterns are reproduced in figures 1 and 2 where types 1, 2, 3 are uniquely characteristic of $\mathrm{Hf} \mathrm{I}$, and $4,5,6$ of $\mathrm{Hf} \mathrm{II}$. This sorting of Hf I and Hf II on the basis of Zeeman types was in perfect agreement with the separation of these spectra according to relative intensities in different light sources.

Besides of great value for sorting spectra, resolved Zeeman patterns are almost indispensable for the quantum interpretation because the quantum numbers $(j)$ associated with the total angular momentum of the radiating atom are given directly by the number of components, and the intervals between neighboring components equal the difference between the magnetic splitting factors $(g)$ of the combining levels. The first observations of Zeeman-effect in hafnium spectra have already been mentioned [6]; they greatly facilitated the analysis of $\mathrm{Hf}$ II, but were inadequate for an extensive analysis of $\mathrm{Hf} 1$ because they were made with a spark operated in a 6-mm pole gap of a Weiss magnet producing a field intensity of 35,000 oersteds. In August 1949 greatly improved Zeeman spectrograms were obtained by courtesy of G. R. Harrison of the Massachusetts Institute of Technology, by using an arc in a magnetic field of 83,000 oersteds produced by the Bitter magnet [12]. The gratings available at MIT also had 2 or 3 times the resolving power of the one employed at the Bureau, but the MIT gratings had very low efficiency for waves longer than about $5000 \mathrm{~A}$. The MIT Zeeman spectrograms of hafnium were therefore supplemented from 5000 to $9000 \mathrm{~A}$ by recent observations at the Bureau with a hafnium-iodide lamp in a magnetic field of 37,000 oersteds. The patterns reproduced in figures 1 and 2 were taken from these spectrograms.

The results of the final effort to provide an improved description of hafnium spectra are presented in table 1 , where measured wavelengths, estimated relative intensities in arc, spark, and tube spectra, assignment of individual lines to successive spectra, and Zeeman types are shown in successive columns. Notation in the table conforms to the report of Subcommittee $e$ of the Joint Commission for Spectroscopy [13]. Further details of the Zeeman effect are reserved for reports on the quantum interpretation of $\mathrm{Hf} \mathrm{I}$ and $\mathrm{Hf} \mathrm{II}$; in the present paper the Zeeman-type numbers suffice to confirm the assignment of particular lines to their proper spectrum as inferred from relative intensities in different light sources.

The generous donations by C. P. Keim and by S. M. Shelton of massive metallic electrodes of hafnium for our investigations of hafnium spectra are gratefully acknowledged by the authors. Also we thank G. R. Harrison for letting us use the excellent equipment at MIT for observing the Zeeman effect.

\section{References}

[1] D. Coster and G. von Hevesy, Nature 111, 79 (1923).

[2] H. M. Hansen and S. Werner, Kgl. Danske Vid Selskab. Math.-fysiske Medd. V. 8 (1923).

[3] W. F. Meggers, BS. J. Research 1, 151 (1928) RP8.

[4] W. F. Meggers and B. F. Scribner, J. Opt. Soc. Am. and Rev. Sci. Instru. 17, 83 (1928).

[5] W. F. Meggers and B. F. Scribner, BS. J. Research 4, 169 (1930) RP139.

[6] W. F. Meggers and B. F. Scribner, J. Research NBS 13, 625 (1934) RP732.

[7] F. P. Venable and J. M. Bell, J. Am. Chem. Soc. 39, 1598 (1917).

[8] F. P. Venable and J. M. Bell, J. Am. Chem. Soc. 46, 1833 (1924).

[9] C. H. Corliss, W. R. Bozman, and F. O. Westfall, J. Opt. Soc. Am. 43, 131 (1953).

[10] W. F. Meggers, J. Research NBS 49, 187 (1952) RP2355.

[11] E. Back and A. Landé, Zeemaneffekt und Multiplettstruktur der Spektrallinien. p. 168 (Julius Springer, Berlin, 1925).

[12] G. R. Harrison and F. Bitter, Phys. Rev. 5\%, 15 (1940).

[13] Trans. of the Joint Commission for Spectroscopy, J. Opt. Soc. Am. 43, 423 (1953). 
TABLE 1. Emission spectra of hafnium

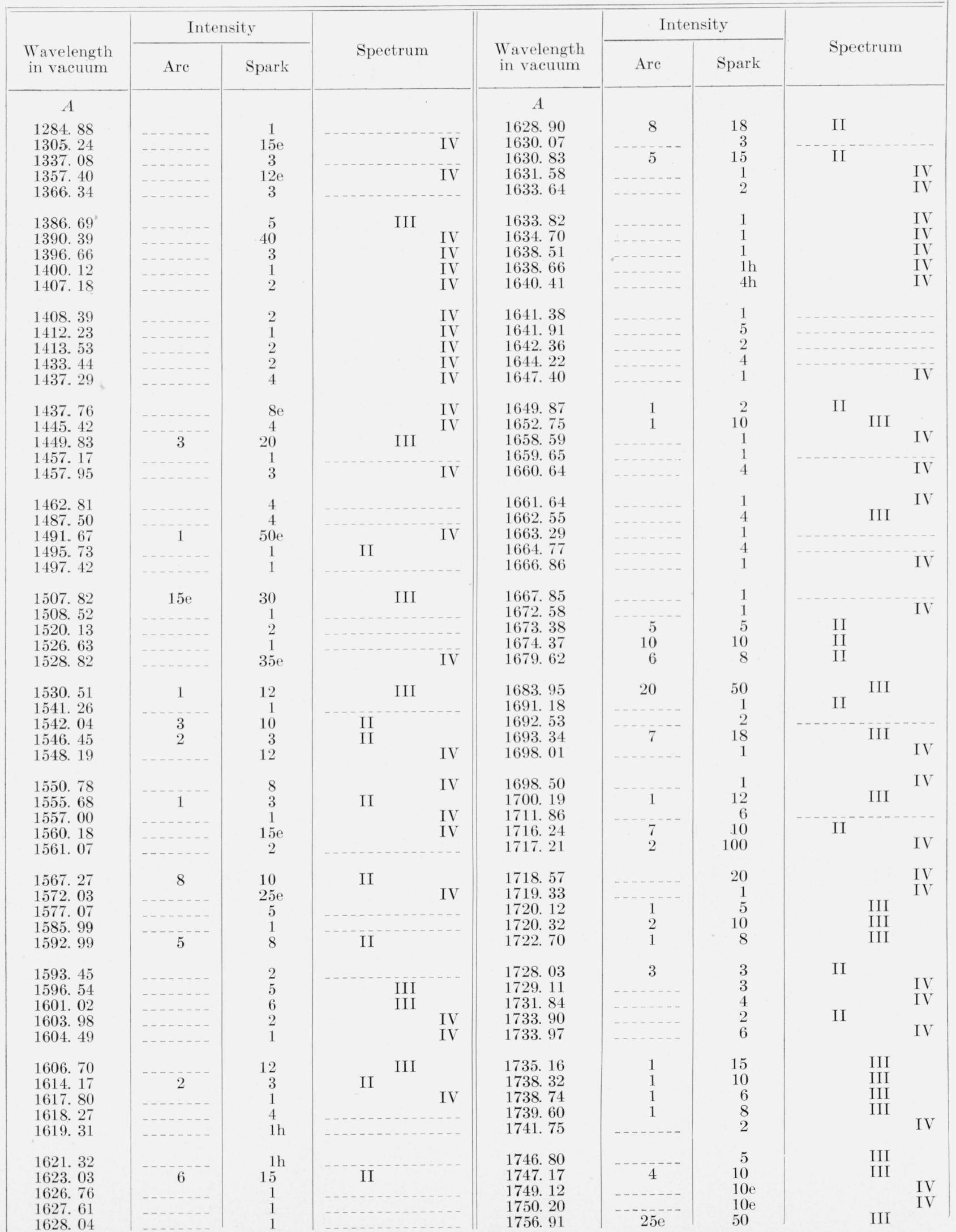


TABLE 1. Emission spectra of hafnium-Continued

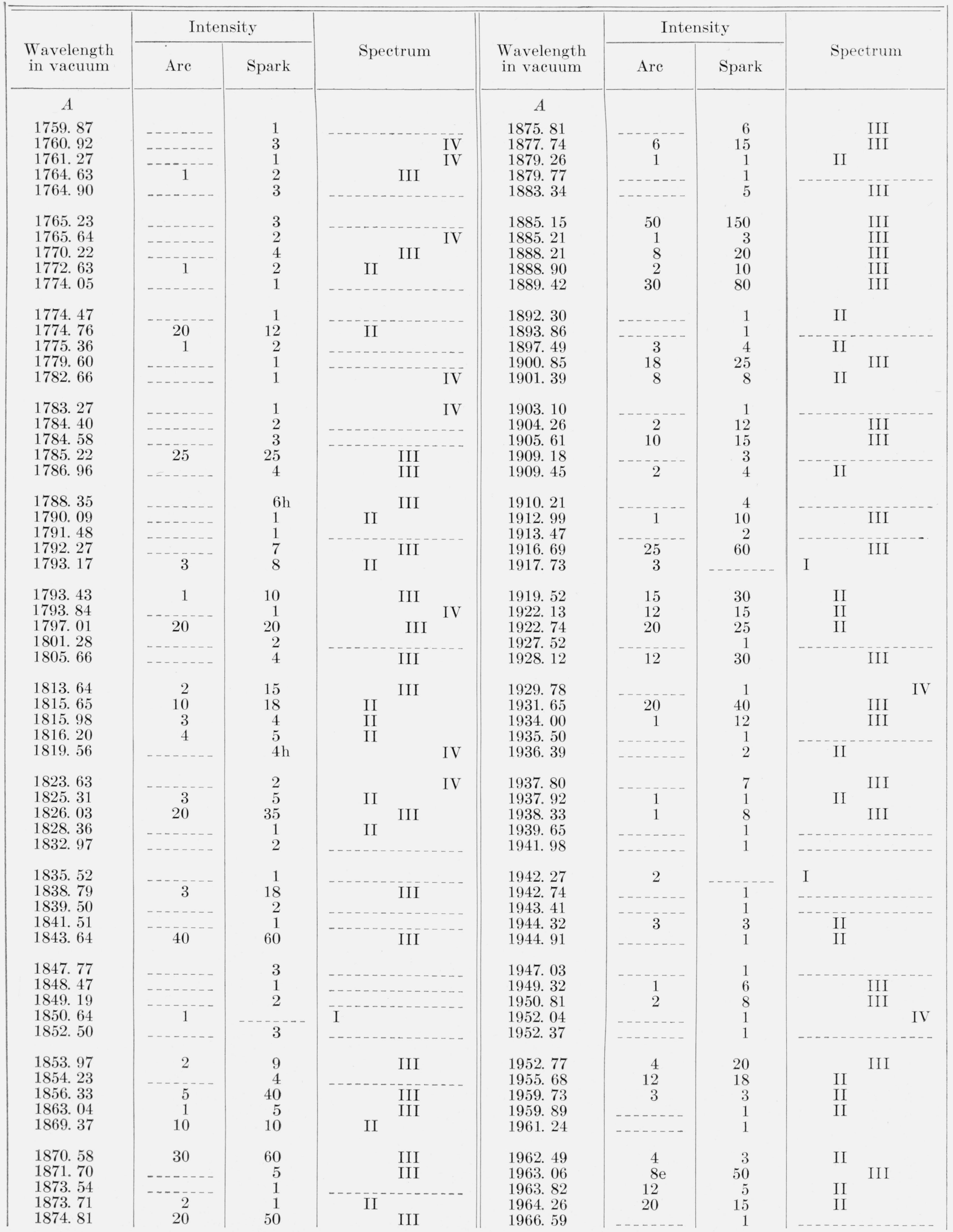


TABLE 1. Emission spectra of hafnium-Continued

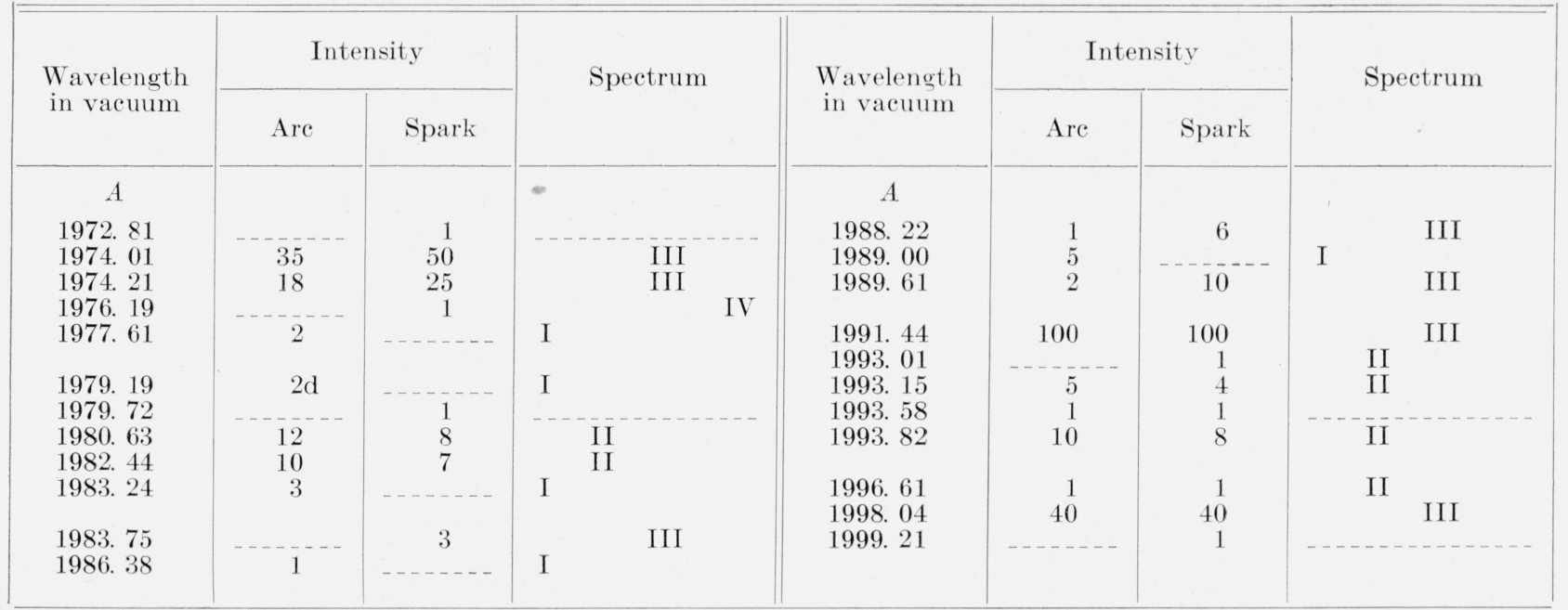

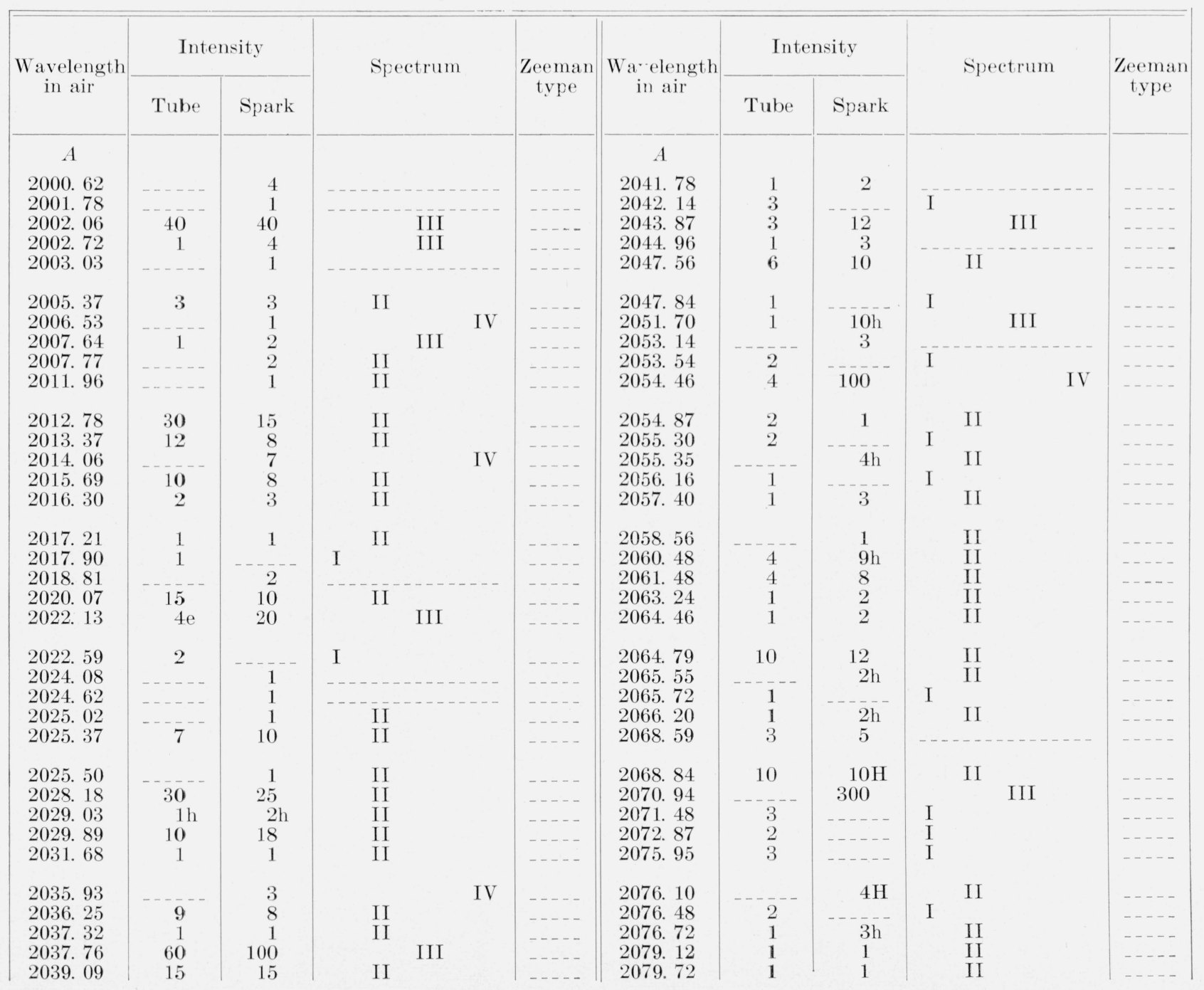


TABLE 1. Emission spectra of hafnium-Continued

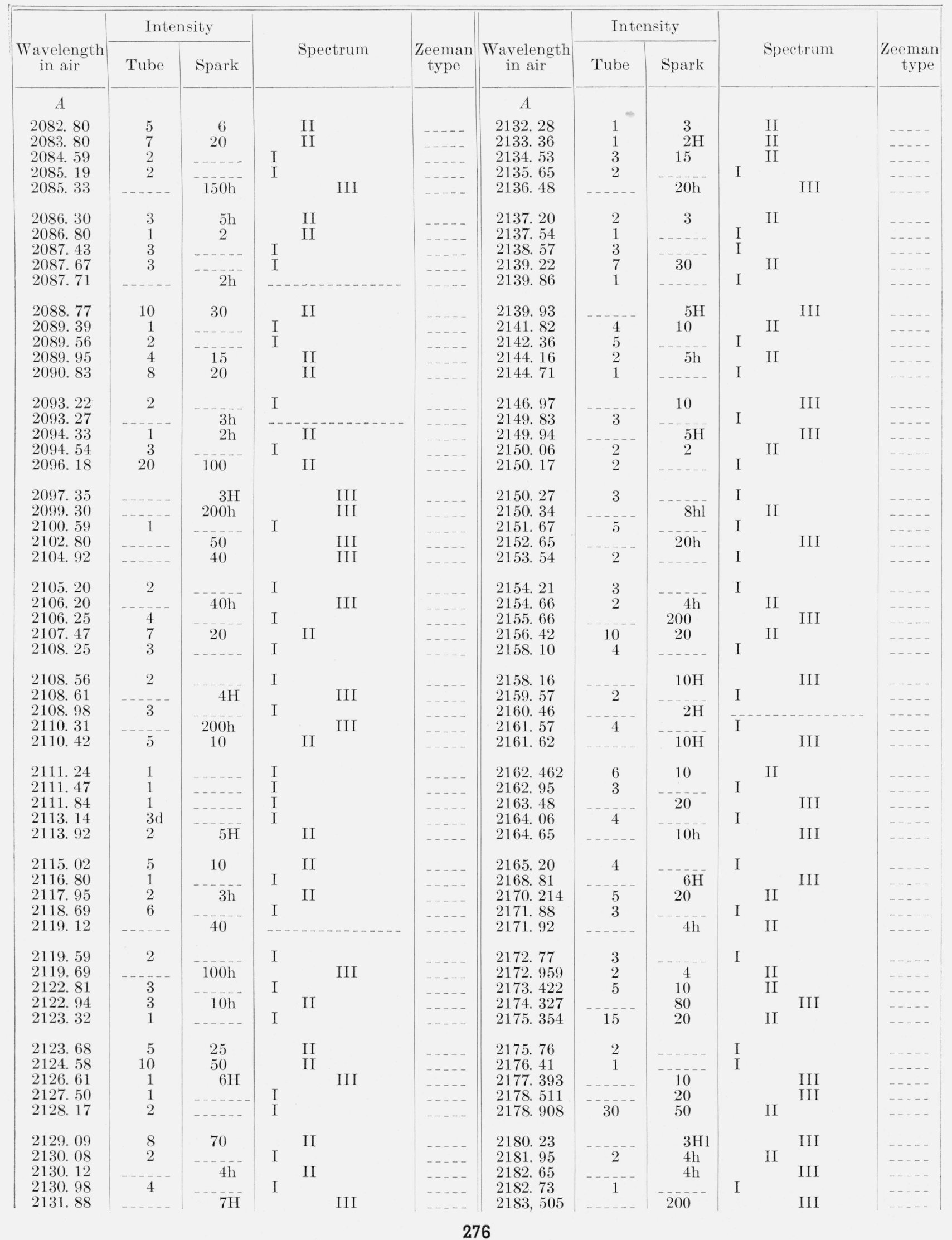


TABLE 1. Emission spectra of hafnium-Continued

\begin{tabular}{|c|c|c|c|c|c|c|c|c|c|c|c|}
\hline \multirow[b]{2}{*}{$\begin{array}{l}\text { Wavelength } \\
\text { in air }\end{array}$} & \multicolumn{2}{|c|}{ Intensity } & \multirow{2}{*}{\multicolumn{2}{|c|}{ Spectrum }} & \multirow[b]{2}{*}{$\begin{array}{c}\text { Zeeman } \\
\text { type }\end{array}$} & \multirow[b]{2}{*}{$\begin{array}{c}\text { Wavelength } \\
\text { in air }\end{array}$} & \multicolumn{2}{|c|}{ Intensity } & \multirow{2}{*}{\multicolumn{2}{|c|}{ Spectrum }} & \multirow[b]{2}{*}{$\begin{array}{l}\text { Zeeman } \\
\text { type }\end{array}$} \\
\hline & Tube & Spark & & & & & Tube & Spark & & & \\
\hline $\begin{array}{c}A \\
\text { 2184. } 30 \\
2184.35 \\
2184.97 \\
2186.29 \\
2187.43\end{array}$ & $\begin{array}{c}3 \\
2 \\
2 \\
2 \\
-\end{array}$ & $\begin{array}{c}10 \mathrm{H} \\
4\end{array}$ & $\begin{array}{l}\text { I } \\
\text { I }\end{array}$ & $\begin{array}{l}\text { II } \\
\text { II }\end{array}$ & $\begin{array}{l}\cdots-1 \\
\cdots- \\
\cdots-- \\
\cdots-\end{array}$ & \begin{tabular}{l}
\multicolumn{1}{c}{$A$} \\
2231. 42 \\
2234.59 \\
2235.13 \\
2235.41 \\
2235. 461
\end{tabular} & $\begin{array}{c}2 \\
2 \\
1 \\
-\end{array}$ & $\begin{array}{c}4 \mathrm{~h} \\
200 \\
10\end{array}$ & $\begin{array}{l}\mathrm{I} \\
\mathrm{I}\end{array}$ & II III & $\begin{array}{l}\cdots- \\
\cdots- \\
\cdots- \\
-\cdots-\end{array}$ \\
\hline $\begin{array}{l}\text { 2187. } 91 \\
2188.428 \\
2188.72 \\
2188.87 \\
2190.212\end{array}$ & $\begin{array}{r}3 \\
3 \\
1 \\
4 \\
10\end{array}$ & $\begin{array}{c}10 \\
20\end{array}$ & $\begin{array}{l}\text { I } \\
\text { I } \\
\text { I }\end{array}$ & $\begin{array}{l}\text { II } \\
\text { II }\end{array}$ & $\begin{array}{l}--\cdots \\
---- \\
---- \\
-\cdots- \\
----\end{array}$ & $\begin{array}{l}2235.635 \\
2236.35 \\
2239.85 \\
2240.20 \\
2241.34\end{array}$ & $\begin{array}{r}4 \\
2 \\
2 \\
1 \\
-\end{array}$ & $\begin{array}{c}3 \mathrm{Hl} \\
3 \mathrm{~h} \\
-8 \mathrm{~h}\end{array}$ & $\begin{array}{l}\mathrm{I} \\
\mathrm{I}\end{array}$ & $\begin{array}{l}\text { II } \\
\text { II }\end{array}$ & $\begin{array}{l}\cdots-1 \\
\cdots- \\
\cdots- \\
\cdots- \\
\cdots-\end{array}$ \\
\hline $\begin{array}{l}\text { 2190. } 388 \\
\text { 2191. } 26 \\
\text { 2191. } 32 \\
\text { 2191. } 78 \\
\text { 2192. } 31\end{array}$ & $\begin{array}{c}1 \\
--- \\
6 \\
2\end{array}$ & $\begin{array}{l}30 \\
3 \mathrm{~h} \\
2 \mathrm{~h} \\
8 \\
3\end{array}$ & & $\begin{array}{ll} & \text { III } \\
\text { II } & \\
\text { II } & \\
\text { II } & \\
\text { II } & \end{array}$ & $\begin{array}{l}--- \\
-\cdots \\
-\cdots- \\
-\cdots- \\
-\cdots\end{array}$ & $\begin{array}{l}2243.16 \\
2243.19 \\
2244.24 \\
2246.29 \\
2246.62\end{array}$ & $\begin{array}{c}5 \\
--- \\
2 \\
1\end{array}$ & $\begin{array}{c}15 \mathrm{hl} \\
-\overline{2 h}\end{array}$ & $\begin{array}{l}\text { I } \\
\text { I } \\
\text { I }\end{array}$ & III & $\begin{array}{l}--- \\
-\cdots \\
-\cdots- \\
-\cdots- \\
-\cdots-\end{array}$ \\
\hline $\begin{array}{l}\text { 2193. } 196 \\
2193.84 \\
2194.12 \\
2194.968 \\
2195.435\end{array}$ & $\begin{array}{r}4 \\
2 \\
1 \\
10 \\
-\end{array}$ & $\begin{array}{l}----- \\
-\cdots--- \\
---\cdots\end{array}$ & $\begin{array}{l}\text { I } \\
\text { I } \\
\text { I } \\
\text { I }\end{array}$ & III & $\begin{array}{l}-\cdots \\
\cdots- \\
\cdots- \\
-\cdots \\
-\cdots\end{array}$ & $\begin{array}{l}2248.20 \\
2248.33 \\
2248.95 \\
2249.01 \\
2249.85\end{array}$ & $\begin{array}{c}1 \\
1 \\
3 \\
3\end{array}$ & $\begin{array}{c}3 \mathrm{~h} \\
5 \overline{\mathrm{H}} \\
7 \mathrm{H}\end{array}$ & $\begin{array}{l}\text { I } \\
\text { I }\end{array}$ & $\begin{array}{l}\text { II } \\
\text { II III }\end{array}$ & $\begin{array}{l}\cdots- \\
\cdots- \\
\cdots- \\
\cdots- \\
\cdots-\end{array}$ \\
\hline $\begin{array}{l}2195.52 \\
2196.03 \\
2196.404 \\
2199.58 \\
2199.84\end{array}$ & $\begin{array}{l}3 \\
4 \\
5 \\
3 \\
2\end{array}$ & $\begin{array}{c}7 \\
5 \mathrm{~h}\end{array}$ & $\begin{array}{l}\text { I } \\
\text { I } \\
\text { I }\end{array}$ & $\begin{array}{l}\text { II } \\
\text { II }\end{array}$ & 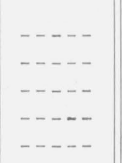 & $\begin{array}{l}2251.85 \\
2253.995 \\
2255.162 \\
2257.894 \\
2258.32\end{array}$ & $\begin{array}{r}3 \\
30 \\
20 \\
4 \\
2\end{array}$ & $\begin{array}{r}6 \\
80 \\
60 \\
10 \\
-\end{array}$ & I & $\begin{array}{l}\text { II } \\
\text { II } \\
\text { II } \\
\text { II }\end{array}$ & 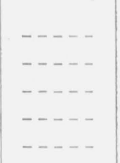 \\
\hline $\begin{array}{l}2201.67 \\
2202.18 \\
2202.97 \\
2203.40 \\
2204.17\end{array}$ & $\begin{array}{l}1 \\
2 \\
1 \\
1 \\
4\end{array}$ & $\begin{array}{c}3 \mathrm{~h} \\
2 \mathrm{~h} \\
-\ldots\end{array}$ & $\begin{array}{l}\mathrm{I} \\
\mathrm{I} \\
\mathrm{I}\end{array}$ & $\begin{array}{l}\text { II } \\
\text { II }\end{array}$ & 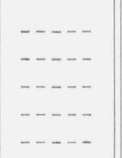 & $\begin{array}{l}2258.685 \\
2260.25 \\
2261.54 \\
2262.015 \\
2262.20\end{array}$ & $\begin{array}{l}5 \\
2 \\
2 \\
5 \\
1\end{array}$ & \begin{tabular}{c}
15 \\
$-\cdots--$ \\
\hdashline---
\end{tabular} & $\begin{array}{l}\text { I } \\
\text { I } \\
\text { I } \\
\text { I }\end{array}$ & II & 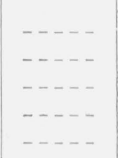 \\
\hline $\begin{array}{l}2204.63 \\
2206.09 \\
2206.11 \\
2210.05 \\
2210.84\end{array}$ & $\begin{array}{c}2 \\
6 \\
-2 \\
2 \\
2\end{array}$ & $\frac{10 \mathrm{H}}{2 \mathrm{~h}}$ & $\begin{array}{l}\mathrm{I} \\
\mathrm{I} \\
\mathrm{I}\end{array}$ & $\begin{array}{l}\text { II } \\
\text { II }\end{array}$ & $\begin{array}{l}-\cdots \\
---- \\
-\cdots- \\
-\cdots \\
-\cdots- \\
-\cdots\end{array}$ & $\begin{array}{l}2263.37 \\
2264.847 \\
2266.521 \\
2266.832 \\
2267.35\end{array}$ & $\begin{array}{c}2 \\
--\overline{10} \\
20 \\
2\end{array}$ & $\begin{array}{c}2 \mathrm{~h} \\
30 \mathrm{~h} \\
15 \\
40 \\
3 \mathrm{~h}\end{array}$ & I & $\begin{array}{ll} & \text { III } \\
\text { II } & \\
\text { II } & \\
\text { II } & \end{array}$ & $\begin{array}{l}\cdots-1 \\
\cdots- \\
\cdots- \\
\cdots-\end{array}$ \\
\hline $\begin{array}{l}\text { 2211. } 32 \\
2211.86 \\
2212.45 \\
2213.54 \\
2214.675\end{array}$ & $\begin{array}{c}2 \\
2 \\
7 \\
-2^{--}\end{array}$ & $\begin{array}{c}3 \\
20 \\
100 \\
----\end{array}$ & I & $\begin{array}{ll}\text { II } & \\
\text { II } & \\
& \text { III }\end{array}$ & $\begin{array}{l}---- \\
---- \\
---1 \\
-\cdots- \\
-\cdots\end{array}$ & $\begin{array}{l}2267.582 \\
2267.91 \\
2268.686 \\
2268.92 \\
2269.483\end{array}$ & $\begin{array}{l}2 \\
2 \\
4 \\
1 \\
3\end{array}$ & 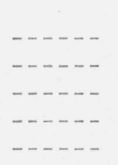 & $\begin{array}{l}\text { I } \\
\text { I } \\
\text { I } \\
\text { I } \\
\text { I }\end{array}$ & & $\begin{array}{l}\cdots- \\
\cdots- \\
-\cdots \\
\cdots- \\
\cdots-\end{array}$ \\
\hline $\begin{array}{l}2214.86 \\
2217.67 \\
2218.36 \\
2218.45 \\
2219.45\end{array}$ & $\begin{array}{l}1 \\
1 \\
1 \\
1\end{array}$ & $\begin{array}{c}15 \\
2 \mathrm{~h} \\
7 \\
\cdots \\
-\end{array}$ & $\begin{array}{l}\mathrm{I} \\
\mathrm{I}\end{array}$ & $\begin{array}{ll} & \text { III } \\
\text { II } & \end{array}$ & $\begin{array}{l}--1 \\
-\cdots \\
-\cdots- \\
\cdots-- \\
-\cdots\end{array}$ & $\begin{array}{l}2269.86 \\
2269.91 \\
2270.69 \\
2271.14 \\
2271.453\end{array}$ & $\begin{array}{c}4 \\
-2^{-} \\
2 \\
5\end{array}$ & $\begin{array}{c}6 \mathrm{hl} \\
4 \\
2 \mathrm{~h}\end{array}$ & $\begin{array}{l}\text { I } \\
\text { I }\end{array}$ & $\begin{array}{ll} & \text { III } \\
\text { II } & \\
\text { II }\end{array}$ & $\begin{array}{l} \\
-\cdots- \\
-\cdots-- \\
-\cdots-- \\
-\cdots- \\
-\cdots-\end{array}$ \\
\hline $\begin{array}{l}2219.50 \\
2219.60 \\
2220.48 \\
2220.55 \\
2221.59\end{array}$ & $\begin{array}{c}1 \\
2 \\
1\end{array}$ & $\begin{array}{r}2 \mathrm{~h} \\
-5 \mathrm{hl} \\
-\end{array}$ & $\begin{array}{l}\mathrm{I} \\
\mathrm{I} \\
\mathrm{I}\end{array}$ & III & $\begin{array}{l}---- \\
-\cdots \\
-\cdots- \\
-\cdots- \\
-\cdots\end{array}$ & $\begin{array}{l}2273.147 \\
2273.92 \\
2274.18 \\
2274.545 \\
2274.64\end{array}$ & $\begin{array}{r}20 \\
3 \\
1 \\
2 \\
4\end{array}$ & $\begin{array}{c}40 \\
-15\end{array}$ & $\begin{array}{l}\text { I } \\
\text { I } \\
\text { I }\end{array}$ & II & $\begin{array}{l}-\cdots- \\
-\cdots- \\
-\cdots- \\
-\cdots- \\
\cdots-\end{array}$ \\
\hline $\begin{array}{l}\text { 2221. } 88 \\
2223.35 \\
2223.94 \\
2224.26 \\
2224.30\end{array}$ & $\begin{array}{c}1 \\
1 \\
6\end{array}$ & $\begin{array}{c}10 \mathrm{~h} \\
2 \\
-10 \overline{\mathrm{H}} \\
5\end{array}$ & $\mathrm{I}$ & $\begin{array}{ll} & \text { III } \\
\text { II } & \\
\text { II } & \\
\text { II } & \end{array}$ & $\begin{array}{l}-\cdots \\
-\cdots- \\
-\cdots- \\
-\cdots- \\
-\cdots\end{array}$ & $\begin{array}{ll}2275 . & 26 \\
2275.483 \\
2276.43 \\
2277.166 \\
2279.195\end{array}$ & $\begin{array}{r}1 \\
6 \\
2 \\
40 \\
4\end{array}$ & $\begin{array}{c}3 \mathrm{~h} \\
150\end{array}$ & $\begin{array}{l}\mathrm{I} \\
\mathrm{I} \\
\mathrm{I}\end{array}$ & $\begin{array}{l}\text { II } \\
\text { II }\end{array}$ & 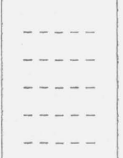 \\
\hline $\begin{array}{l}2225.352 \\
2228.180 \\
2228.45 \\
2228.772 \\
2230.35\end{array}$ & $\begin{array}{l}5 \\
5 \\
3 \\
3 \\
4\end{array}$ & $\begin{array}{l}6 \\
6 \\
3 \mathrm{~h} \\
5 \\
-\end{array}$ & I & $\begin{array}{l}\text { II } \\
\text { II } \\
\text { II } \\
\text { II }\end{array}$ & 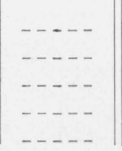 & $\begin{array}{l}2280.072 \\
2281.683 \\
2281.82 \\
2282.80 \\
2283.089\end{array}$ & $\begin{array}{l}4 \\
5 \\
1 \\
3 \\
5\end{array}$ & $3 \mathrm{~h}$ & $\begin{array}{l}\mathrm{I} \\
\mathrm{I} \\
\mathrm{I} \\
\mathrm{I}\end{array}$ & II & 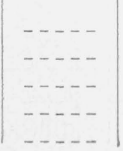 \\
\hline
\end{tabular}


TABLE 1. Emission spectra of hafnium-Continued

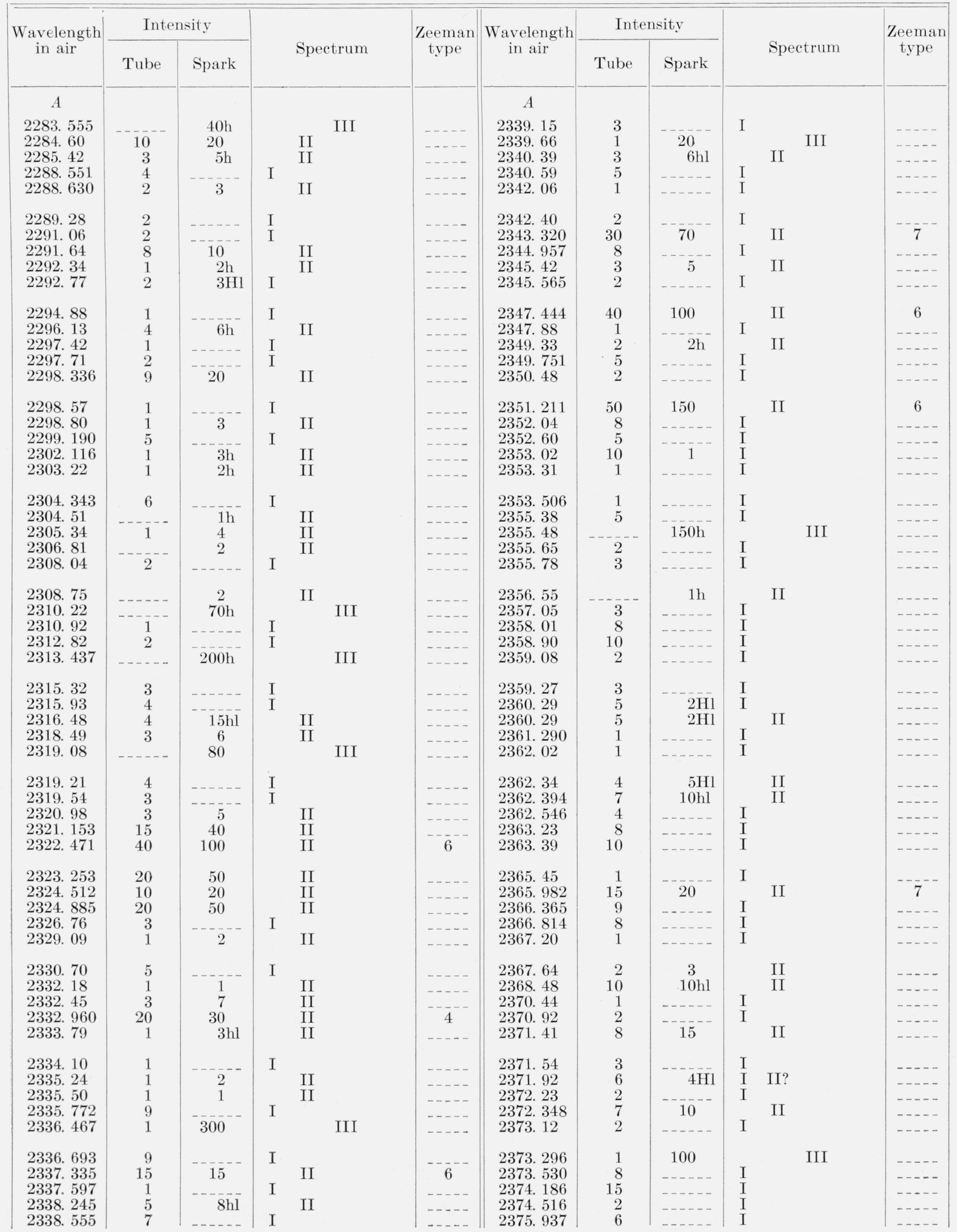


TABLE 1. Emission spectra of hafnium-Continued

\begin{tabular}{|c|c|c|c|c|c|c|c|c|c|c|c|}
\hline \multirow{2}{*}{$\begin{array}{l}\text { Wavelength } \\
\text { in air }\end{array}$} & \multicolumn{2}{|c|}{ Intensity } & \multirow{2}{*}{\multicolumn{2}{|c|}{ Spectrum }} & \multirow{2}{*}{$\begin{array}{c}\text { Zeeman } \\
\text { type }\end{array}$} & \multirow{2}{*}{$\begin{array}{l}\text { Wavelength } \\
\text { in air }\end{array}$} & \multicolumn{2}{|c|}{ Intensity } & \multirow{2}{*}{\multicolumn{2}{|c|}{ Spectrum }} & \multirow{2}{*}{$\begin{array}{c}\text { Zeeman } \\
\text { type }\end{array}$} \\
\hline & Tube & Spark & & & & & Tube & Spark & & & \\
\hline \begin{tabular}{l}
\multicolumn{1}{c}{. } \\
2376. 46 \\
2377.237 \\
2377.471 \\
2377.57 \\
2377.98
\end{tabular} & $\begin{array}{l}1 \\
2 \\
4 \\
1 \\
3\end{array}$ & 120 & $\begin{array}{l}\text { I } \\
\text { I } \\
\text { I } \\
\text { I }\end{array}$ & III & $\begin{array}{l}-\cdots- \\
-\cdots- \\
-\cdots- \\
-\cdots- \\
----\end{array}$ & \begin{tabular}{l}
\multicolumn{1}{c}{$A$} \\
2410.137 \\
2410.97 \\
2411.14 \\
2411.425 \\
2411.605
\end{tabular} & $\begin{array}{r}200 \\
3 \\
2 \\
8 \\
15\end{array}$ & $\begin{array}{c}300 \\
-3 \mathrm{hl} \\
-3\end{array}$ & $\begin{array}{l}\text { I } \\
\text { I } \\
\text { I }\end{array}$ & $\begin{array}{l}\text { II } \\
\text { II }\end{array}$ & $\begin{array}{c}6 \\
---- \\
--- \\
-\cdots \\
-\cdots\end{array}$ \\
\hline $\begin{array}{l}2378.163 \\
2378.32 \\
2378.513 \\
2378.81 \\
2378.925\end{array}$ & $\begin{array}{r}15 \\
1 \\
1 \\
4 \\
4\end{array}$ & 3 hi & $\begin{array}{l}\text { I } \\
\text { I } \\
\text { I } \\
\text { I } \\
\text { I }\end{array}$ & II & $\begin{array}{l}-\cdots-- \\
---- \\
-\cdots-- \\
---- \\
----\end{array}$ & $\begin{array}{l}\text { 2412. } 036 \\
2412.261 \\
2412.51 \\
2412.622 \\
2412.85\end{array}$ & $\begin{array}{l}4 \\
2 \\
7 \\
6 \\
4\end{array}$ & \begin{tabular}{c}
$5 \mathrm{~h}$ \\
$-\cdots$ \\
\hdashline--- \\
$-\cdots$
\end{tabular} & $\begin{array}{l}\text { I } \\
\text { I } \\
\text { I } \\
\text { I }\end{array}$ & II & $\begin{array}{l}-\cdots- \\
-\cdots- \\
-\cdots- \\
-\cdots-\end{array}$ \\
\hline $\begin{array}{l}\text { 2379. } 716 \\
\text { 2380. } 305 \\
\text { 2381. } 002 \\
\text { 2381. } 51 \\
\text { 2382. } 047\end{array}$ & $\begin{array}{r}5 \\
50 \\
30 \\
1 \\
2\end{array}$ & $\begin{array}{r}100 \\
60 \\
1 \\
3\end{array}$ & I & $\begin{array}{l}\text { II } \\
\text { II } \\
\text { II } \\
\text { II }\end{array}$ & $\begin{array}{c}-\overline{7}^{--} \\
7 \\
-\end{array}$ & $\begin{array}{l}\text { 2413. } 348 \\
2413.871 \\
2414.045 \\
2415.11 \\
2415.59\end{array}$ & $\begin{array}{r}20 \\
4 \\
1 \\
2 \\
3\end{array}$ & \begin{tabular}{c}
60 \\
\hdashline $4 \mathrm{Hl}$ \\
--
\end{tabular} & $\begin{array}{l}\mathrm{I} \\
\mathrm{I} \\
\mathrm{I}\end{array}$ & $\begin{array}{l}\text { II } \\
\text { II }\end{array}$ & $\begin{array}{l}-\cdots \\
-\cdots- \\
-\cdots \\
-\cdots-\end{array}$ \\
\hline $\begin{array}{l}2382.980 \\
2383.540 \\
2384.467 \\
2385.180 \\
2385.79\end{array}$ & $\begin{array}{r}15 \\
1 \\
15 \\
3 \\
2\end{array}$ & $\begin{array}{c}250 \\
4 \\
3\end{array}$ & $\begin{array}{l}\text { I } \\
\text { I }\end{array}$ & $\begin{array}{ll} & \text { III } \\
\text { II } & \\
\text { II } & \end{array}$ & $\begin{array}{l}-\cdots- \\
---- \\
-\cdots- \\
----\end{array}$ & $\begin{array}{l}2415.72 \\
2415.84 \\
2415.965 \\
2416.427 \\
2416.504\end{array}$ & $\begin{array}{r}5 \\
4 \\
15 \\
15 \\
6\end{array}$ & $\begin{array}{c}30 \\
1\end{array}$ & $\begin{array}{l}\text { I } \\
\text { I } \\
\text { I } \\
\text { I }\end{array}$ & II & $\begin{array}{c}--- \\
-\cdots \\
-\cdots\end{array}$ \\
\hline $\begin{array}{l}\text { 2386. } 014 \\
\text { 2386. } 33 \\
\text { 2386. } 562 \\
\text { 2386. } 70 \\
\text { 2387. } 38\end{array}$ & $\begin{array}{l}1 \\
3 \\
2 \\
1 \\
5\end{array}$ & $6 \mathrm{Hl}$ & $\begin{array}{l}\text { I } \\
\text { I } \\
\text { I } \\
\text { I }\end{array}$ & II & $\begin{array}{l}---- \\
-\cdots- \\
-\cdots- \\
-\cdots- \\
----\end{array}$ & $\begin{array}{l}\text { 2416. } 886 \\
\text { 2417. } 030 \\
2417.26 \\
2417.690 \\
\text { 2418. } 074\end{array}$ & $\begin{array}{c}3 \\
4 \\
-200 \\
- \\
2\end{array}$ & $\begin{array}{c}5 \mathrm{Hl} \\
-20 \\
300 \\
-\end{array}$ & $\begin{array}{l}\mathrm{I} \\
\mathrm{I}\end{array}$ & $\begin{array}{l}\text { II } \\
\text { II III }\end{array}$ & $-\bar{y}$ \\
\hline $\begin{array}{l}\text { 2387. } 89 \\
\text { 2388. 50 } \\
\text { 2390. } 20 \\
\text { 2390. } 794 \\
\text { 2392. 008 }\end{array}$ & $\begin{array}{l}2 \\
4 \\
8 \\
3\end{array}$ & $\begin{array}{c}6 \mathrm{~h} \\
4 \mathrm{hl}\end{array}$ & $\begin{array}{l}\text { I } \\
\text { I } \\
\text { I }\end{array}$ & III & $\begin{array}{l}--- \\
-\cdots \\
-\cdots- \\
-\cdots \\
----\end{array}$ & $\begin{array}{l}\text { 2418. } 29 \\
2418.76 \\
2419.17 \\
2419.371 \\
2420.08\end{array}$ & $\begin{array}{l}1 \\
1 \\
2 \\
2 \\
1\end{array}$ & 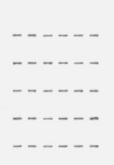 & $\begin{array}{l}\text { I } \\
\text { I } \\
\text { I } \\
\text { I } \\
\text { I }\end{array}$ & & $\begin{array}{l}-\cdots- \\
-\cdots \\
-\cdots- \\
-\cdots-\end{array}$ \\
\hline $\begin{array}{l}2393.185 \\
2393.37 \\
2393.828 \\
2394.973 \\
2395.625\end{array}$ & $\begin{array}{r}40 \\
80 \\
100 \\
2 \\
2\end{array}$ & $\begin{array}{r}60 \\
100 \\
150 \\
-3\end{array}$ & I & $\begin{array}{l}\text { II } \\
\text { II } \\
\text { II } \\
\text { II }\end{array}$ & $\begin{array}{c}4 \\
4 \\
5 \\
- \\
-\end{array}$ & $\begin{array}{l}2420.22 \\
2420.554 \\
2420.78 \\
2422.09 \\
2422.856\end{array}$ & $\begin{array}{r}2 \\
2 \\
3 \\
20 \\
4\end{array}$ & $\begin{array}{r}5 \mathrm{hl} \\
-\overline{9 \mathrm{hl}} \\
-\overline{5} \overline{\mathrm{hl}}\end{array}$ & $\begin{array}{l}\text { I } \\
\text { I }\end{array}$ & $\begin{array}{l}\text { II } \\
\text { II } \\
\text { II }\end{array}$ & $\begin{array}{l}-\ldots- \\
-\cdots \\
-\cdots- \\
-\cdots-\end{array}$ \\
\hline $\begin{array}{l}\text { 2396. } 02 \\
\text { 2396. } 98 \\
2397.21 \\
\text { 2397. } 565 \\
\text { 2397. } 780\end{array}$ & $\begin{array}{r}1 \\
4 \\
2 \\
10 \\
20\end{array}$ & $\begin{array}{c}1 \\
-1 \\
-1-1- \\
-1-\end{array}$ & $\begin{array}{l}\text { I } \\
\text { I } \\
\text { I } \\
\text { I }\end{array}$ & II & 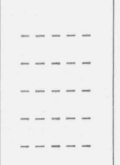 & $\begin{array}{l}2423.73 \\
2424.003 \\
2424.92 \\
2425.975 \\
2426.52\end{array}$ & $\begin{array}{r}2 \\
15 \\
1 \\
100 \\
5\end{array}$ & $\begin{array}{r}4 \mathrm{hl} \\
30 \mathrm{hl} \\
-200 \\
-\end{array}$ & $\begin{array}{l}\text { I } \\
\text { I }\end{array}$ & $\begin{array}{l}\text { II } \\
\text { II } \\
\text { II }\end{array}$ & $\frac{--}{-\cdots}$ \\
\hline $\begin{array}{l}\text { 2398. } 121 \\
\text { 2399. } 144 \\
\text { 2399. } 726 \\
\text { 2400. } 325 \\
\text { 2400. } 804\end{array}$ & $\begin{array}{r}10 \\
6 \\
20 \\
2 \\
30\end{array}$ & $\begin{array}{l}-1 . \\
-80\end{array}$ & $\begin{array}{l}\text { I } \\
\text { I } \\
\text { I } \\
\text { I }\end{array}$ & II & $\frac{---}{-\cdots}$ & $\begin{array}{l}2426.804 \\
2426.86 \\
2428.740 \\
2428.993 \\
2430.144\end{array}$ & $\begin{array}{r}9 \\
7 \\
30 \\
50 \\
20\end{array}$ & $\begin{array}{c}20 \mathrm{hl} \\
200 \\
3\end{array}$ & $\begin{array}{l}\text { I } \\
\text { I } \\
\text { I }\end{array}$ & $\begin{array}{l}\text { II } \\
\text { II } \\
\text { II }\end{array}$ & $\frac{--}{-\cdots}$ \\
\hline $\begin{array}{l}\text { 2401. } 31 \\
\text { 2401. } 522 \\
\text { 2402. } 02 \\
\text { 2403. } 12 \\
\text { 2403. } 296\end{array}$ & $\begin{array}{l}2 \\
1 \\
4 \\
8 \\
4\end{array}$ & $\begin{array}{c}2 \mathrm{~h} \\
10 \mathrm{hl} \\
\cdots\end{array}$ & $\begin{array}{l}\text { I } \\
\text { I } \\
\text { I }\end{array}$ & $\begin{array}{l}\text { II } \\
\text { II }\end{array}$ & $\begin{array}{l}---1 \\
-\cdots-1 \\
-\cdots-1 \\
-\cdots-1\end{array}$ & $\begin{array}{l}2430.92 \\
2431.152 \\
2431.414 \\
2432.303 \\
2432.854\end{array}$ & $\begin{array}{l}2 \\
1 \\
5 \\
2 \\
1\end{array}$ & 3 h & $\begin{array}{l}\text { I } \\
\text { I } \\
\text { I } \\
\text { I }\end{array}$ & II & $\begin{array}{l}-\cdots- \\
-\cdots-1 \\
---1- \\
-\cdots-\end{array}$ \\
\hline $\begin{array}{l}2403.606 \\
2404.566 \\
2405.421 \\
2405.679 \\
2406.051\end{array}$ & $\begin{array}{r}10 \\
24 \\
250 \\
3 \\
5\end{array}$ & $\begin{array}{r}20 \\
60 \\
400 \\
-\end{array}$ & $\begin{array}{l}\mathrm{I} \\
\mathrm{I}\end{array}$ & $\begin{array}{l}\text { II } \\
\text { II } \\
\text { II }\end{array}$ & $\begin{array}{c}5 \\
6 \\
4 \\
--- \\
---\end{array}$ & $\begin{array}{l}\text { 2433. } 216 \\
2433.564 \\
2433.798 \\
2433.88 \\
2434.266\end{array}$ & $\begin{array}{r}2 \\
60 \\
9 \\
1 \\
2\end{array}$ & $\begin{array}{c}300 \\
2 \\
-\end{array}$ & $\begin{array}{l}\mathrm{I} \\
\mathrm{I} \\
\mathrm{I}\end{array}$ & $\begin{array}{l}\text { II } \\
\text { II }\end{array}$ & $\begin{array}{c}---- \\
4 \\
-\cdots- \\
----\end{array}$ \\
\hline $\begin{array}{l}2406.440 \\
2407.132 \\
2407.692 \\
2409.140 \\
2409.54\end{array}$ & $\begin{array}{r}50 \\
9 \\
20 \\
2 \\
2\end{array}$ & \begin{tabular}{l}
100 \\
\hdashline-1 \\
-0
\end{tabular} & $\begin{array}{l}\text { I } \\
\text { I } \\
\text { I } \\
\text { I }\end{array}$ & II & 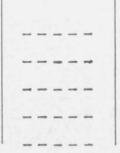 & $\begin{array}{l}2434.522 \\
2434.764 \\
2435.472 \\
2435.767 \\
2436.16\end{array}$ & $\begin{array}{r}1 \\
30 \\
3 \\
2 \\
7\end{array}$ & $\begin{array}{c}80 \\
10 \mathrm{hl}\end{array}$ & $\begin{array}{l}\mathrm{I} \\
\mathrm{I} \\
\mathrm{I}\end{array}$ & $\begin{array}{l}\text { II } \\
\text { II }\end{array}$ & $\mid$\begin{tabular}{c}
$--\overline{7}^{--}$ \\
$-\cdots$ \\
\hdashline--
\end{tabular} \\
\hline
\end{tabular}


TABLE 1. Emission spectra of hafnium-Continued

\begin{tabular}{|c|c|c|c|c|c|c|c|c|c|c|c|}
\hline \multirow{2}{*}{$\begin{array}{c}\text { Wavelength } \\
\text { in air }\end{array}$} & \multicolumn{2}{|c|}{ Intensity } & \multirow{2}{*}{\multicolumn{2}{|c|}{ Spectrum }} & \multirow{2}{*}{$\begin{array}{c}\text { Zeeman } \\
\text { type }\end{array}$} & \multirow{2}{*}{$\begin{array}{l}\text { Wavelength } \\
\text { in air }\end{array}$} & \multicolumn{2}{|c|}{ Intensity } & & \multirow{2}{*}{ Spectrum } & \multirow{2}{*}{$\begin{array}{l}\text { Zeeman } \\
\text { type }\end{array}$} \\
\hline & Tube & Spark & & & & & Tube & Spark & & & \\
\hline \begin{tabular}{l}
\multicolumn{1}{c}{$A$} \\
2437.00 \\
2437.79 \\
2438.316 \\
2438.535 \\
2440.814
\end{tabular} & $\begin{array}{r}2 \\
3 \\
5 \\
9 \\
15\end{array}$ & 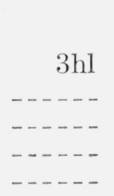 & $\begin{array}{l}\text { I } \\
\text { I } \\
\text { I } \\
\text { I }\end{array}$ & II & $\begin{array}{l}\cdots- \\
\cdots- \\
\cdots- \\
\cdots- \\
\cdots-\end{array}$ & \begin{tabular}{c}
\multicolumn{1}{c}{$A$} \\
2469.179 \\
2469.965 \\
2470.998 \\
2471.21 \\
2471.77
\end{tabular} & $\begin{array}{r}150 \\
15 \\
20 \\
10 \\
6\end{array}$ & $\begin{array}{r}500 \\
-5 \mathrm{Hl} \\
6 \mathrm{Hl}\end{array}$ & $\begin{array}{l}\text { I } \\
\text { I } \\
\text { I }\end{array}$ & $\begin{array}{l}\text { II } \\
\text { II } \\
\text { II }\end{array}$ & \begin{tabular}{c}
4 \\
-1 \\
\hdashline-
\end{tabular} \\
\hline $\begin{array}{l}2441.05 \\
2441.22 \\
2441.28 \\
2441.53 \\
2441.920\end{array}$ & \begin{tabular}{c}
20 \\
1 \\
\hdashline 1 \\
30
\end{tabular} & \begin{tabular}{c}
$40 \mathrm{hl}$ \\
\hdashline 10 \\
-1
\end{tabular} & $\begin{array}{l}\text { I } \\
\text { I } \\
\text { I }\end{array}$ & $\begin{array}{l}\text { II } \\
\text { II }\end{array}$ & $\begin{array}{l}-\ldots- \\
-\cdots \\
-\cdots \\
-\cdots- \\
-\cdots-\end{array}$ & $\begin{array}{l}2471.84 \\
2471.92 \\
2472.54 \\
2472.89 \\
2473.00\end{array}$ & $\begin{array}{l}7 \\
6 \\
1 \\
1\end{array}$ & $\begin{array}{c}1 \mathrm{~h} \\
\cdots \\
\cdots\end{array}$ & $\begin{array}{l}\text { I } \\
\text { I } \\
\text { I } \\
\text { I }\end{array}$ & $\begin{array}{l}\text { II } \\
\text { II }\end{array}$ & $\begin{array}{l}-\cdots- \\
-\cdots- \\
-\cdots- \\
-\cdots- \\
----\end{array}$ \\
\hline $\begin{array}{l}2443.27 \\
2443.766 \\
2444.994 \\
2445.58 \\
2446.248\end{array}$ & $\begin{array}{r}2 \\
6 \\
50 \\
4 \\
2\end{array}$ & \begin{tabular}{c}
$5 \mathrm{hl}$ \\
\hdashline 1 \\
$9 \mathrm{Hl}$ \\
$\ldots$
\end{tabular} & $\begin{array}{l}\text { I } \\
\text { I } \\
\text { I }\end{array}$ & $\begin{array}{l}\text { II } \\
\text { II }\end{array}$ & 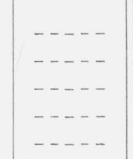 & $\begin{array}{l}2473.736 \\
2473.908 \\
2474.089 \\
2475.573 \\
2476.05\end{array}$ & $\begin{array}{r}4 \\
50 \\
15 \\
9 \\
6\end{array}$ & $\begin{array}{c}200 \\
30 \\
10 \mathrm{hl}\end{array}$ & I & $\begin{array}{l}\text { II } \\
\text { II } \\
\text { II }\end{array}$ & $\begin{array}{c}4 \\
--- \\
-\cdots--\end{array}$ \\
\hline $\begin{array}{l}2446.731 \\
2447.251 \\
2447.65 \\
2448.216 \\
2448.624\end{array}$ & $\begin{array}{r}7 \\
200 \\
1 \\
\hdashline 1\end{array}$ & \begin{tabular}{c}
$3 \mathrm{hl}$ \\
500 \\
\hdashline $15 \mathrm{~h}$ \\
-
\end{tabular} & $\begin{array}{l}\mathrm{I} \\
\mathrm{I} \\
\mathrm{I}\end{array}$ & $\begin{array}{ll}\text { II } & \\
\text { II } & \\
& \text { III }\end{array}$ & 6 & $\begin{array}{l}2476.30 \\
2476.36 \\
2476.92 \\
2478.542 \\
2479.076\end{array}$ & $\begin{array}{r}1 \\
3 \\
3 \\
20 \\
3\end{array}$ & $\begin{array}{c}2 \mathrm{~h} \\
4 \mathrm{Hl} \\
50\end{array}$ & $\begin{array}{l}\text { I } \\
\text { I }\end{array}$ & $\begin{array}{l}\text { II } \\
\text { II } \\
\text { II }\end{array}$ & $\frac{1}{5}$ \\
\hline $\begin{array}{l}2449.053 \\
2449.440 \\
2450.05 \\
2450.080 \\
2450.66\end{array}$ & $\begin{array}{r}6 \\
80 \\
4 \\
10 \\
1\end{array}$ & $\begin{array}{c}200 \\
5 \mathrm{hl} \\
\cdots\end{array}$ & $\begin{array}{l}\text { I } \\
\text { I } \\
\text { I }\end{array}$ & $\begin{array}{l}\text { II } \\
\text { II }\end{array}$ & 6 & $\begin{array}{l}2479.428 \\
2479.76 \\
2480.57 \\
2481.436 \\
2481.96\end{array}$ & $\begin{array}{r}20 \\
1 \\
1 \\
30 \\
3\end{array}$ & $\begin{array}{c}100 \\
3 \mathrm{~h}\end{array}$ & $\begin{array}{l}\text { I } \\
\text { I } \\
\text { I }\end{array}$ & $\begin{array}{l}\text { II } \\
\text { II }\end{array}$ & $\ldots$ \\
\hline $\begin{array}{l}2451.885 \\
2452.296 \\
2452.474 \\
2453.013 \\
2453.336\end{array}$ & $\begin{array}{c}20 \\
20 \\
-10 \\
30 \\
60\end{array}$ & $\begin{array}{c}40 \\
80 \\
300\end{array}$ & $\begin{array}{l}\text { I } \\
\text { I }\end{array}$ & $\begin{array}{ll}\text { II } & \text { III } \\
\text { II } & \end{array}$ & 4 & $\begin{array}{l}2482.010 \\
2482.518 \\
2482.647 \\
2482.934 \\
2483.346\end{array}$ & $\begin{array}{r}20 \\
3 \\
40 \\
15 \\
50\end{array}$ & $\begin{array}{c}5 \\
2 \\
80\end{array}$ & $\begin{array}{l}\mathrm{I} \\
\mathrm{I} \\
\mathrm{I}\end{array}$ & $\begin{array}{l}\text { II } \\
\text { II }\end{array}$ & $\frac{1}{5}$ \\
\hline $\begin{array}{l}2453.990 \\
2455.200 \\
2455.711 \\
2456.061 \\
2456.31\end{array}$ & $\begin{array}{r}20 \\
10 \\
1 \\
30 \\
1\end{array}$ & 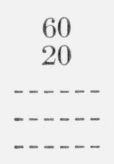 & $\begin{array}{l}\text { I } \\
\text { I } \\
\text { I }\end{array}$ & $\begin{array}{l}\text { II } \\
\text { II }\end{array}$ & $\begin{array}{c}6 \\
6 \\
-.- \\
-\cdots\end{array}$ & $\begin{array}{l}\text { 2484. } 990 \\
2485.354 \\
2485.555 \\
2485.83 \\
2486.09\end{array}$ & $\begin{array}{r}10 \\
3 \\
20 \\
3 \\
3\end{array}$ & $\begin{array}{l}8 \mathrm{Hl} \\
4 \mathrm{hl}\end{array}$ & $\begin{array}{l}\text { I } \\
\text { I } \\
\text { I }\end{array}$ & $\begin{array}{l}\text { II } \\
\text { II }\end{array}$ & $\begin{array}{l}-\cdots \\
-\cdots \\
-\cdots \\
\cdots-- \\
-\cdots\end{array}$ \\
\hline $\begin{array}{l}2456.65 \\
2456.957 \\
2457.871 \\
2458.653 \\
2459.470\end{array}$ & $\begin{array}{r}8 \\
4 \\
15 \\
30 \\
15\end{array}$ & \begin{tabular}{c}
$10 \mathrm{Hl}$ \\
\hdashline$-{ }^{-}$
\end{tabular} & $\begin{array}{l}\text { I } \\
\text { I } \\
\text { I }\end{array}$ & II & $\begin{array}{l}-\cdots \\
\cdots \cdots \\
\cdots \cdots \\
\cdots \cdots \\
\cdots-1\end{array}$ & $\begin{array}{l}2487.153 \\
2487.90 \\
2488.14 \\
2488.867 \\
2489.236\end{array}$ & $\begin{array}{r}60 \\
3 \\
4 \\
6 \\
15\end{array}$ & \begin{tabular}{c}
${ }_{3}^{2} \mathrm{Hl}$ \\
$-\cdots$ \\
\hdashline..--
\end{tabular} & $\begin{array}{l}\text { I } \\
\text { I } \\
\text { I } \\
\text { I }\end{array}$ & II & $\begin{array}{l}\cdots- \\
\cdots- \\
\cdots- \\
\cdots-\end{array}$ \\
\hline $\begin{array}{l}2460.493 \\
2461.12 \\
2461.602 \\
2461.74 \\
2462.76\end{array}$ & $\begin{array}{r}200 \\
1 \\
3 \\
-9\end{array}$ & \begin{tabular}{c}
500 \\
\hdashline 400 \\
$10 \mathrm{Hl}$
\end{tabular} & $\begin{array}{l}\mathrm{I} \\
\mathrm{I}\end{array}$ & $\begin{array}{ll}\text { II } & \\
& \text { III }\end{array}$ & \begin{tabular}{c}
6 \\
$-\cdots$ \\
\hdashline$-\cdots$ \\
--
\end{tabular} & $\begin{array}{l}2489.892 \\
2490.237 \\
2490.650 \\
2490.89 \\
2491.058\end{array}$ & $\begin{array}{l}2 \\
3 \\
2 \\
9 \\
3\end{array}$ & $\begin{array}{c}4 \mathrm{~h} \\
30 \mathrm{Hl}\end{array}$ & $\begin{array}{l}\mathrm{I} \\
\mathrm{I} \\
\mathrm{I}\end{array}$ & $\begin{array}{l}\text { II } \\
\text { II }\end{array}$ & $\begin{array}{l}-\cdots- \\
-\cdots- \\
-\cdots \\
-\cdots- \\
-\cdots-\end{array}$ \\
\hline $\begin{array}{l}2462.926 \\
2463.378 \\
2463.547 \\
2463.725 \\
2463.933\end{array}$ & $\begin{array}{r}4 \\
3 \\
6 \\
3 \\
40\end{array}$ & $\begin{array}{l}-\ldots- \\
-100\end{array}$ & $\begin{array}{l}\text { I } \\
\text { I } \\
\text { I } \\
\text { I }\end{array}$ & II & $\begin{array}{c}-\ldots \\
\cdots-\ldots \\
6\end{array}$ & $\begin{array}{l}\text { 2491. } 156 \\
\text { 2491. } 64 \\
\text { 2491. } 871 \\
\text { 2492. } 919 \\
\text { 2493. } 06\end{array}$ & $\begin{array}{l}2 \\
1 \\
2 \\
3 \\
5\end{array}$ & $3 \mathrm{~h}$ & $\begin{array}{l}\text { I } \\
\text { I } \\
\text { I }\end{array}$ & II & $\begin{array}{l}-\cdots \\
\cdots- \\
-\cdots \\
-\cdots \\
-\cdots\end{array}$ \\
\hline $\begin{array}{l}2464.193 \\
2464.83 \\
2465.059 \\
2465.46 \\
2465.676\end{array}$ & $\begin{array}{r}200 \\
1 \\
50 \\
1 \\
30\end{array}$ & $\begin{array}{c}600 \\
-150 \\
1\end{array}$ & $\begin{array}{l}\text { I } \\
\text { I } \\
\text { I }\end{array}$ & $\begin{array}{l}\text { II } \\
\text { II }\end{array}$ & $\begin{array}{c}7 \\
\cdots \cdots \\
\cdots \cdots\end{array}$ & $\begin{array}{l}\text { 2493. } 25 \\
2493.61 \\
2494.364 \\
2494.74 \\
2495.16\end{array}$ & $\begin{array}{r}1 \\
30 \\
2 \\
5\end{array}$ & $\begin{array}{c}3 \mathrm{~h} \\
40 \mathrm{Hl} \\
2000\end{array}$ & $\begin{array}{l}\mathrm{I} \\
\mathrm{I}\end{array}$ & $\begin{array}{l}\text { II } \\
\text { II }\end{array}$ & 6 \\
\hline $\begin{array}{l}2466.464 \\
2466.665 \\
2467.518 \\
2467.966 \\
2468.378\end{array}$ & $\begin{array}{r}8 \\
10 \\
6 \\
60 \\
15\end{array}$ & 100 & $\begin{array}{l}\text { I } \\
\text { I } \\
\text { I } \\
\text { I }\end{array}$ & II & 5 & $\begin{array}{l}\text { 2495. } 675 \\
2496.058 \\
2496.818 \\
2496.991 \\
2497.730\end{array}$ & $\begin{array}{r}1 \\
40 \\
30 \\
100 \\
8\end{array}$ & 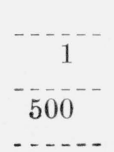 & $\begin{array}{l}\text { I } \\
\text { I } \\
\text { I } \\
\text { I }\end{array}$ & II & 5 \\
\hline
\end{tabular}


TABLE 1. Emission spectra of hafnium-Continued

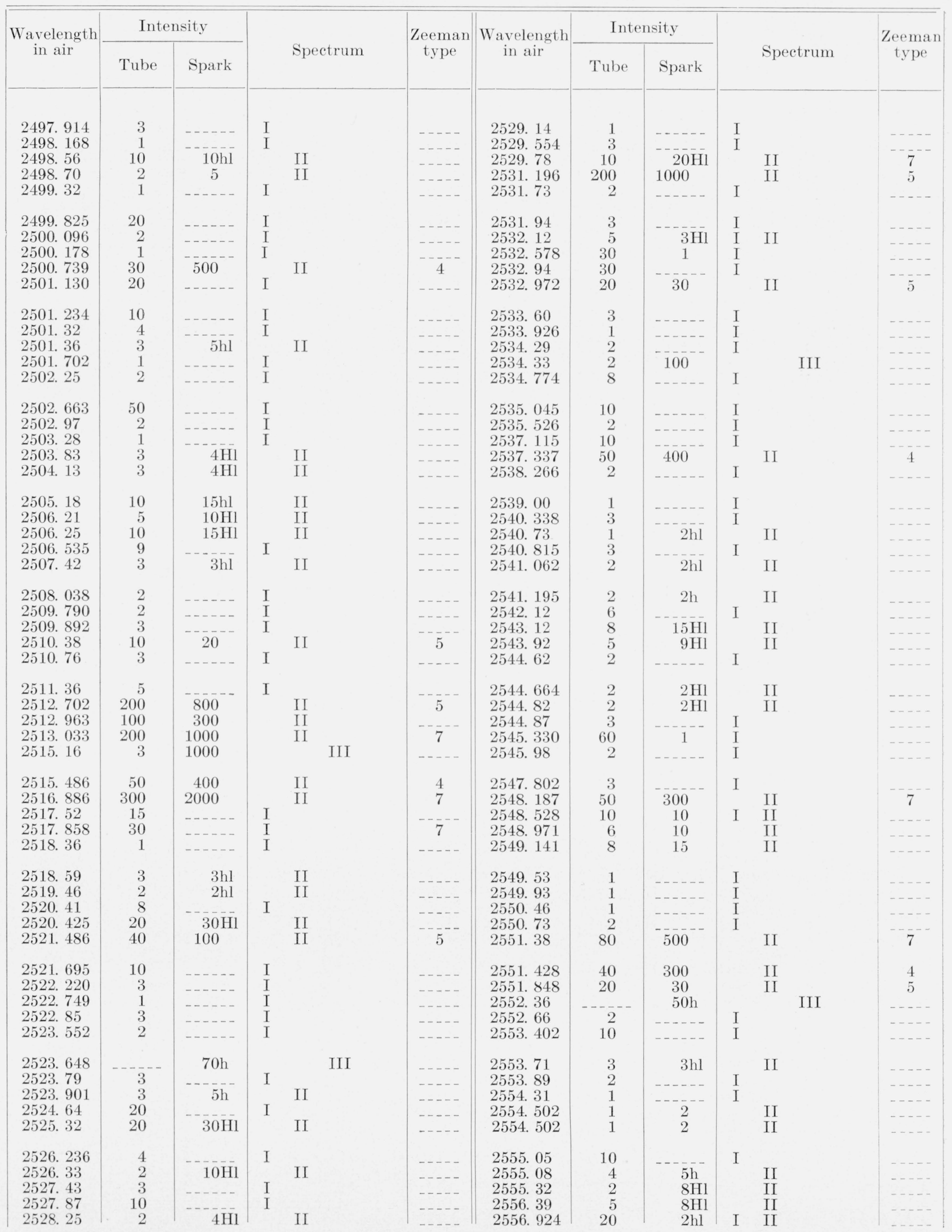


TABLE 1. Emission spectra of hafnium-Continued

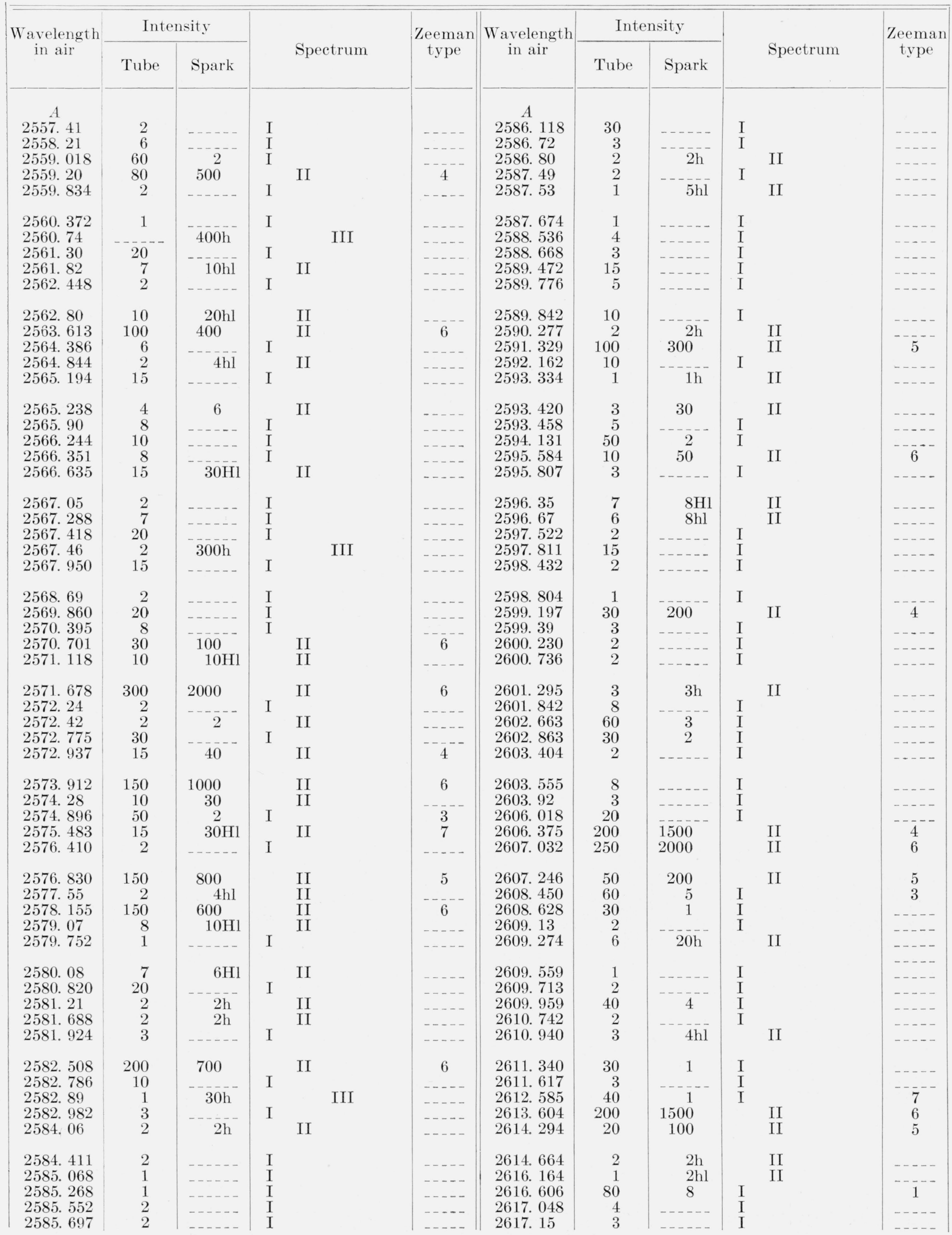


TABLE 1. Emission spectra of hafnium-Continued

\begin{tabular}{|c|c|c|c|c|c|c|c|c|c|c|c|}
\hline \multirow{2}{*}{$\begin{array}{c}\text { Wavelength } \\
\text { in air }\end{array}$} & \multicolumn{2}{|c|}{ Intensity } & \multirow{2}{*}{\multicolumn{2}{|c|}{ Spectrum }} & \multirow{2}{*}{$\begin{array}{c}\text { Zeeman } \\
\text { type }\end{array}$} & \multirow{2}{*}{$\begin{array}{l}\text { Wavelength } \\
\text { in air }\end{array}$} & \multicolumn{2}{|c|}{ Intensity } & \multirow{2}{*}{\multicolumn{2}{|c|}{ Spectrum }} & \multirow{2}{*}{$\begin{array}{c}\text { Zeeman } \\
\text { type }\end{array}$} \\
\hline & Tube & Spark & & & & & Tube & Spark & & & \\
\hline \begin{tabular}{l}
\multicolumn{1}{c}{$A$} \\
2617. 649 \\
2617.804 \\
2618.424 \\
2619.46 \\
2620.33
\end{tabular} & $\begin{array}{l}2 \\
5 \\
2 \\
2 \\
1\end{array}$ & $\begin{array}{r}3 \mathrm{hl} \\
\ldots \ldots--- \\
-\ldots--- \\
-\ldots \ldots-\end{array}$ & $\begin{array}{l}\text { I } \\
\text { I } \\
\text { I } \\
\text { I }\end{array}$ & II & $\begin{array}{l}\cdots-1 \\
\cdots- \\
\cdots- \\
\cdots-\end{array}$ & \begin{tabular}{l}
\multicolumn{1}{c}{$A$} \\
2649.440 \\
2649.864 \\
2650.022 \\
2650.28 \\
2650.737
\end{tabular} & $\begin{array}{r}4 \\
3 \\
2 \\
-1\end{array}$ & 50 & $\begin{array}{l}\text { I } \\
\text { I } \\
\text { I } \\
\text { I }\end{array}$ & III & $\begin{array}{l}-\cdots \\
-\cdots \\
-\cdots \\
-\cdots-\end{array}$ \\
\hline $\begin{array}{l}\text { 2620. } 466 \\
2620.590 \\
2620.772 \\
2620.930 \\
2621.781\end{array}$ & $\begin{array}{r}3 \\
20 \\
10 \\
9 \\
15\end{array}$ & $\begin{array}{l}---- \\
--5^{--} \\
20 \mathrm{hl}\end{array}$ & $\begin{array}{l}\text { I } \\
\text { I } \\
\text { I }\end{array}$ & $\begin{array}{l}\text { II } \\
\text { II }\end{array}$ & $\begin{array}{c}-\cdots- \\
-\cdots \\
7 \\
-\cdots\end{array}$ & $\begin{array}{l}2651.166 \\
2651.785 \\
2652.330 \\
2652.781 \\
2653.040\end{array}$ & $\begin{array}{r}100 \\
3 \\
6 \\
50 \\
2\end{array}$ & $\begin{array}{c}300 \\
20 \\
1 \\
4 \mathrm{Hl}\end{array}$ & $\begin{array}{l}\mathrm{I} \\
\mathrm{I}\end{array}$ & $\begin{array}{l}\text { II } \\
\text { II } \\
\text { II }\end{array}$ & $\begin{array}{c}7 \\
6 \\
-\end{array}$ \\
\hline $\begin{array}{l}2622.739 \\
2623.318 \\
2623.665 \\
2623.906 \\
2624.308\end{array}$ & $\begin{array}{r}400 \\
40 \\
4 \\
3 \\
2\end{array}$ & $\begin{array}{c}2000 \\
1 \\
\ldots \ldots \\
\ldots \ldots \\
\ldots \ldots\end{array}$ & $\begin{array}{l}\text { I } \\
\text { I } \\
\text { I } \\
\text { I }\end{array}$ & II & $\begin{array}{c}5 \\
---- \\
----- \\
----- \\
-----\end{array}$ & $\begin{array}{l}2653.826 \\
2654.30 \\
2654.35 \\
2655.03 \\
2655.394\end{array}$ & $\begin{array}{r}30 \\
1 \\
1 \\
3 \\
4\end{array}$ & $\begin{array}{l}1 \\
1 \mathrm{hl} \\
2 \mathrm{~h} \\
5\end{array}$ & $\begin{array}{l}\mathrm{I} \\
\mathrm{I} \\
\mathrm{I}\end{array}$ & $\begin{array}{l}\text { II } \\
\text { II }\end{array}$ & 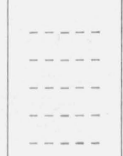 \\
\hline $\begin{array}{l}2624.576 \\
2624.79 \\
2625.533 \\
2626.081 \\
2626.948\end{array}$ & $\begin{array}{r}5 \\
60 \\
20 \\
1 \\
50\end{array}$ & $\begin{array}{c}50 \\
40\end{array}$ & $\begin{array}{l}\mathrm{I} \\
\mathrm{I} \\
\mathrm{I}\end{array}$ & $\begin{array}{l}\text { II } \\
\text { II } \\
\text { II }\end{array}$ & ${ }^{-}--$ & $\begin{array}{l}2655.657 \\
2655.68 \\
2656.95 \\
2657.487 \\
2657.847\end{array}$ & $\begin{array}{r}3 \\
--2 \\
50 \\
100\end{array}$ & $\begin{array}{l}2 \mathrm{~h} \\
100 \\
200\end{array}$ & $\begin{array}{l}\mathrm{I} \\
\mathrm{I}\end{array}$ & $\begin{array}{l}\text { II } \\
\text { II } \\
\text { II }\end{array}$ & $\begin{array}{l}4 \\
5\end{array}$ \\
\hline $\begin{array}{l}2627.720 \\
2627.805 \\
2628.353 \\
2629.490 \\
2629.756\end{array}$ & $\begin{array}{r}1 \\
1 \\
1 \\
10 \\
3\end{array}$ & $1 \mathrm{~h}$ & $\begin{array}{l}\mathrm{I} \\
\mathrm{I} \\
\mathrm{I}\end{array}$ & $\begin{array}{l}\text { II } \\
\text { II }\end{array}$ & $\begin{array}{l}---- \\
---- \\
---- \\
---- \\
-\cdots--\end{array}$ & $\begin{array}{l}2659.130 \\
2659.25 \\
2659.704 \\
2660.523 \\
2661.15\end{array}$ & $\begin{array}{r}5 \\
5 \\
-5 \\
3 \\
20\end{array}$ & $\begin{array}{c}5 \mathrm{~h} \\
60 \\
3 \mathrm{~h}\end{array}$ & $\mathrm{I}$ & $\begin{array}{ll}\text { II } & \\
\text { II }\end{array}$ & $\begin{array}{l}-\cdots \\
-\cdots \\
-\cdots- \\
-\cdots \\
-\cdots\end{array}$ \\
\hline $\begin{array}{l}2629.983 \\
2630.168 \\
2630.34 \\
2630.907 \\
2631.518\end{array}$ & $\begin{array}{r}8 \\
9 \\
2 \\
100 \\
1\end{array}$ & $\begin{array}{c}15 \mathrm{~h} \\
-3 \\
2\end{array}$ & $\begin{array}{l}\text { I } \\
\text { I } \\
\text { I }\end{array}$ & $\begin{array}{l}\text { II } \\
\text { II }\end{array}$ & $\begin{array}{l}---- \\
---- \\
-\cdots- \\
-\cdots- \\
-\cdots--\end{array}$ & $\begin{array}{l}2661.883 \\
2662.396 \\
2662.994 \\
2663.572 \\
2663.717\end{array}$ & $\begin{array}{r}200 \\
15 \\
30 \\
3 \\
10\end{array}$ & 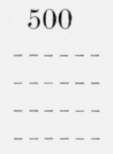 & $\begin{array}{l}\text { I } \\
\text { I } \\
\text { I } \\
\text { I }\end{array}$ & II & $\begin{array}{c}5 \\
--- \\
-\cdots \\
-\cdots\end{array}$ \\
\hline $\begin{array}{ll}2632 . & 017 \\
2632 . & 737 \\
2633 . & 42 \\
2633 . & 504 \\
2634 . & 248\end{array}$ & $\begin{array}{l}6 \\
1 \\
2 \mathrm{~d} \\
2 \\
30\end{array}$ & $\begin{array}{c}20 \mathrm{~h} \\
-\cdots-- \\
-\ldots-- \\
-\ldots- \\
\ldots-\end{array}$ & $\begin{array}{l}\text { I } \\
\text { I } \\
\text { I } \\
\text { I }\end{array}$ & II & $\begin{array}{l}----- \\
----- \\
----- \\
----- \\
-----\end{array}$ & $\begin{array}{l}\text { 2664. } 02 \\
\text { 2664. } 583 \\
2664.786 \\
\text { 2665. } 051 \\
\text { 2665. } 974\end{array}$ & $\begin{array}{r}6 \\
1 \\
5 \\
9 \\
100\end{array}$ & $\begin{array}{c}2 \mathrm{~h} \\
-\overline{----} \\
-\overline{-} \\
-\overline{-}\end{array}$ & $\begin{array}{l}\text { I } \\
\text { I } \\
\text { I } \\
\text { I }\end{array}$ & II & $\frac{-1}{7}$ \\
\hline $\begin{array}{l}\text { 2634. } 425 \\
2635.104 \\
2635.570 \\
2635.780 \\
2636.686\end{array}$ & $\begin{array}{r}20 \\
3 \\
40 \\
60 \\
4\end{array}$ & $\begin{array}{c}1 \\
300\end{array}$ & $\begin{array}{l}\text { I } \\
\text { I } \\
\text { I } \\
\text { I }\end{array}$ & II & $\begin{array}{c}---- \\
---- \\
---- \\
----\end{array}$ & $\begin{array}{l}\text { 2666. } 93 \\
2667.12 \\
2667.514 \\
2668.290 \\
2669.003\end{array}$ & $\begin{array}{r}4 \\
2 \\
20 \\
90 \\
80\end{array}$ & $\begin{array}{c}3 \mathrm{~h} \\
3 \mathrm{~h} \\
20 \mathrm{hl} \\
6 \\
200\end{array}$ & I & $\begin{array}{l}\text { II } \\
\text { II } \\
\text { II } \\
\text { II }\end{array}$ & $\begin{array}{l}1 \\
6\end{array}$ \\
\hline $\begin{array}{l}\text { 2636. } 997 \\
2637.510 \\
2637.853 \\
\text { 2638. } 712 \\
\text { 2638. } 975\end{array}$ & $\begin{array}{r}50 \\
2 \\
15 \\
200 \\
-\end{array}$ & $\begin{array}{c}5 \\
---- \\
400 \\
80\end{array}$ & $\begin{array}{l}\text { I } \\
\text { I } \\
\text { I }\end{array}$ & II III & $\begin{array}{c}-\cdots \\
-\cdots \\
-\cdots\end{array}$ & $\begin{array}{l}2669.558 \\
2669.924 \\
2670.305 \\
2670.638 \\
2670.76\end{array}$ & $\begin{array}{l}8 \\
2 \\
3 \\
1 \\
2\end{array}$ & $\begin{array}{c}20 \\
---- \\
-\cdots\end{array}$ & $\begin{array}{l}\mathrm{I} \\
\mathrm{I} \\
\mathrm{I}\end{array}$ & $\begin{array}{l}\text { II } \\
\text { II }\end{array}$ & 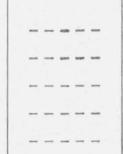 \\
\hline $\begin{array}{l}\text { 2639. } 183 \\
2639.960 \\
2640.121 \\
2640.829 \\
2641.410\end{array}$ & $\begin{array}{r}40 \\
3 \\
1 \\
4 \\
300\end{array}$ & $\begin{array}{l}----- \\
----- \\
-1000\end{array}$ & $\begin{array}{l}\text { I } \\
\text { I } \\
\text { I } \\
\text { I }\end{array}$ & II & $\begin{array}{c}---- \\
----- \\
---- \\
----\end{array}$ & $\begin{array}{l}\text { 2670. } 895 \\
\text { 2671. } 022 \\
2671.24 \\
\text { 2671. } 721 \\
\text { 2672. } 122\end{array}$ & $\begin{array}{r}6 \\
2 \\
30 \\
40 \\
3\end{array}$ & $\begin{array}{c}100 \\
1\end{array}$ & $\begin{array}{l}\mathrm{I} \\
\mathrm{I} \\
\mathrm{I} \\
\mathrm{I}\end{array}$ & II & 6 \\
\hline $\begin{array}{l}\text { 2642. } 076 \\
2642.751 \\
2643.886 \\
2643.999 \\
2644.443\end{array}$ & $\begin{array}{r}60 \\
80 \\
4 \\
15 \\
4\end{array}$ & $\begin{array}{c}2 \\
6 \\
--- \\
\frac{1}{5 \mathrm{Hl}}\end{array}$ & $\begin{array}{l}\text { I } \\
\text { I } \\
\text { I } \\
\text { I }\end{array}$ & II & $\begin{array}{c}2 \\
2 \\
---- \\
---- \\
----\end{array}$ & $\begin{array}{l}2672.454 \\
2673.007 \\
2673.060 \\
2673.191 \\
2673.542\end{array}$ & $\begin{array}{l}1 \\
9 \\
5 \\
2 \\
1\end{array}$ & $\begin{array}{l}-\cdots- \\
-\cdots- \\
-\cdots- \\
-\cdots-1 \\
-\cdots-1\end{array}$ & $\begin{array}{l}\text { I } \\
\text { I } \\
\text { I } \\
\text { I } \\
\text { I }\end{array}$ & & $\begin{array}{l}\cdots-1 \\
\cdots-1 \\
-\cdots \\
-\cdots\end{array}$ \\
\hline $\begin{array}{l}\text { 2645. } 262 \\
2647.294 \\
2648.045 \\
2649.136 \\
2649.397\end{array}$ & $\begin{array}{r}20 \\
200 \\
5 \\
30 \\
2\end{array}$ & $\begin{array}{c}1 \\
600 \\
5 \mathrm{hl} \\
100\end{array}$ & I & $\begin{array}{l}\text { II } \\
\text { II } \\
\text { II }\end{array}$ & $\frac{6}{5}$ & $\begin{array}{l}2673.78 \\
2674.50 \\
2674.80 \\
2675.250 \\
2675.47\end{array}$ & $\begin{array}{r}1 \\
1 \\
5 \\
10 \\
8\end{array}$ & $\begin{array}{r}4 \mathrm{hl} \\
2 \mathrm{~h}\end{array}$ & $\begin{array}{l}\mathrm{I} \\
\mathrm{I} \\
\mathrm{I} \\
\mathrm{I}\end{array}$ & II & $\begin{array}{l}\cdots-1 \\
\cdots-1 \\
\cdots-1 \\
\cdots-1\end{array}$ \\
\hline
\end{tabular}


TABLE 1. Emission spectra of hafnium-Continued

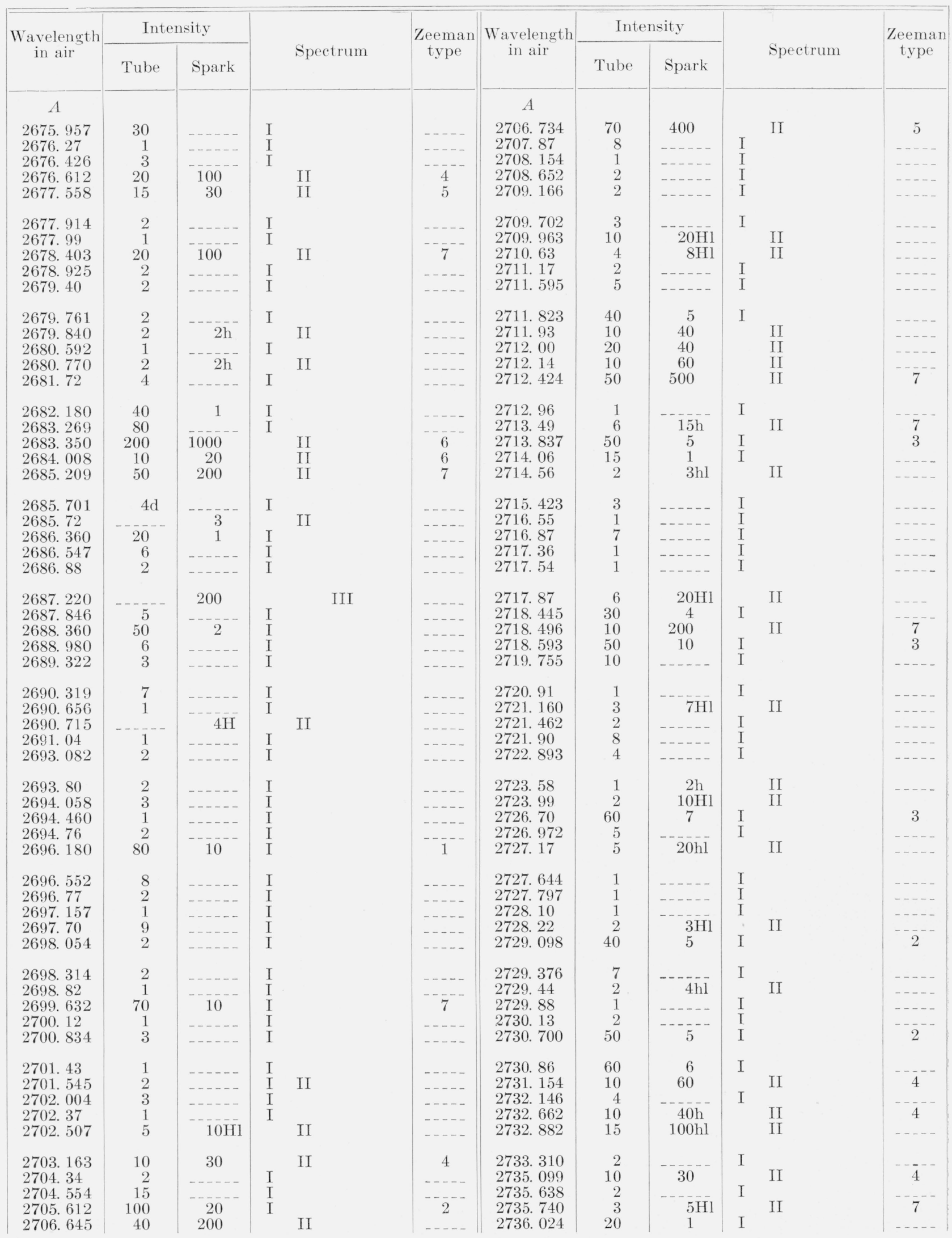


TABLe 1. Emission spectra of hafnium-Continued

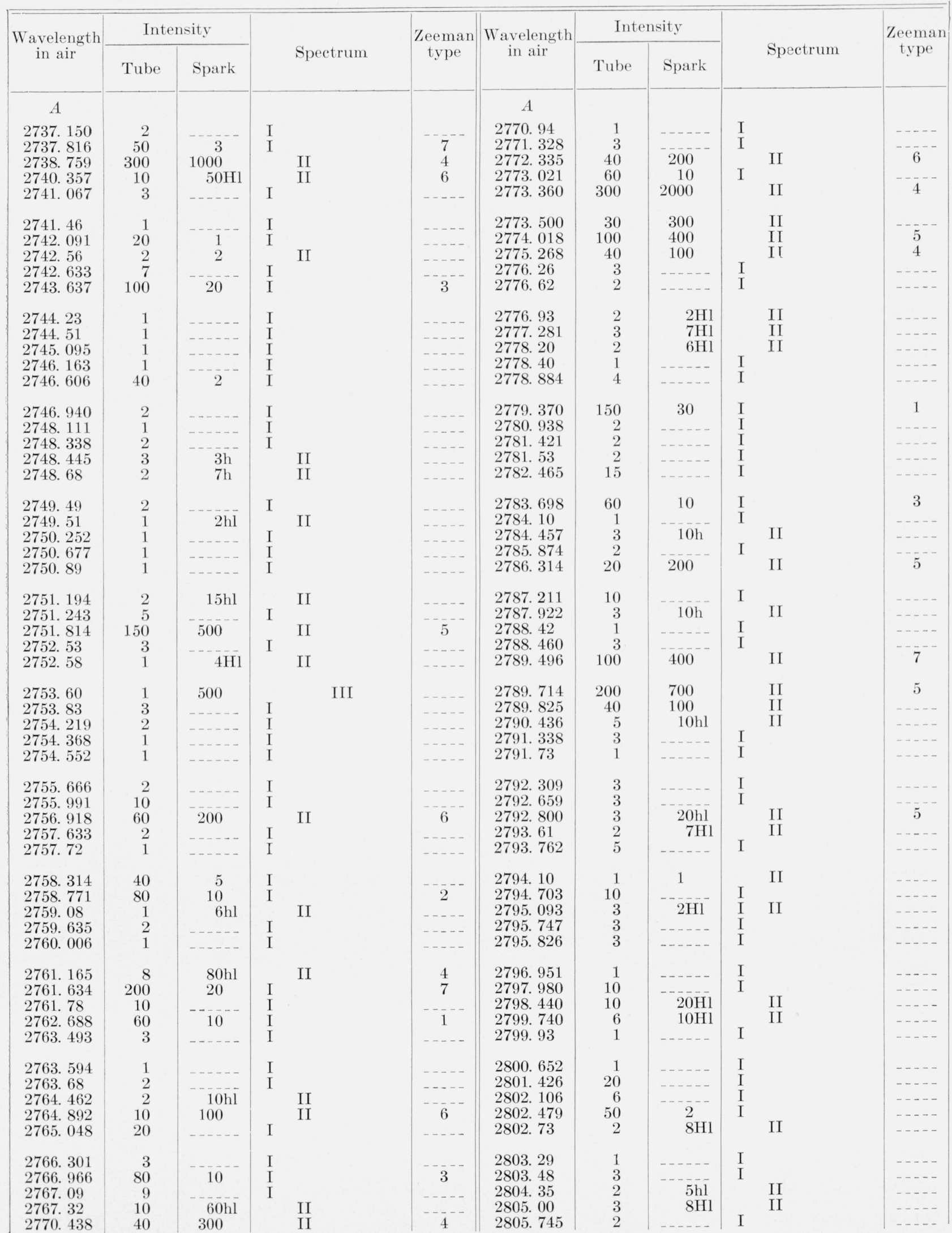


TABLE 1. Emission spectra of hafnium-Continued

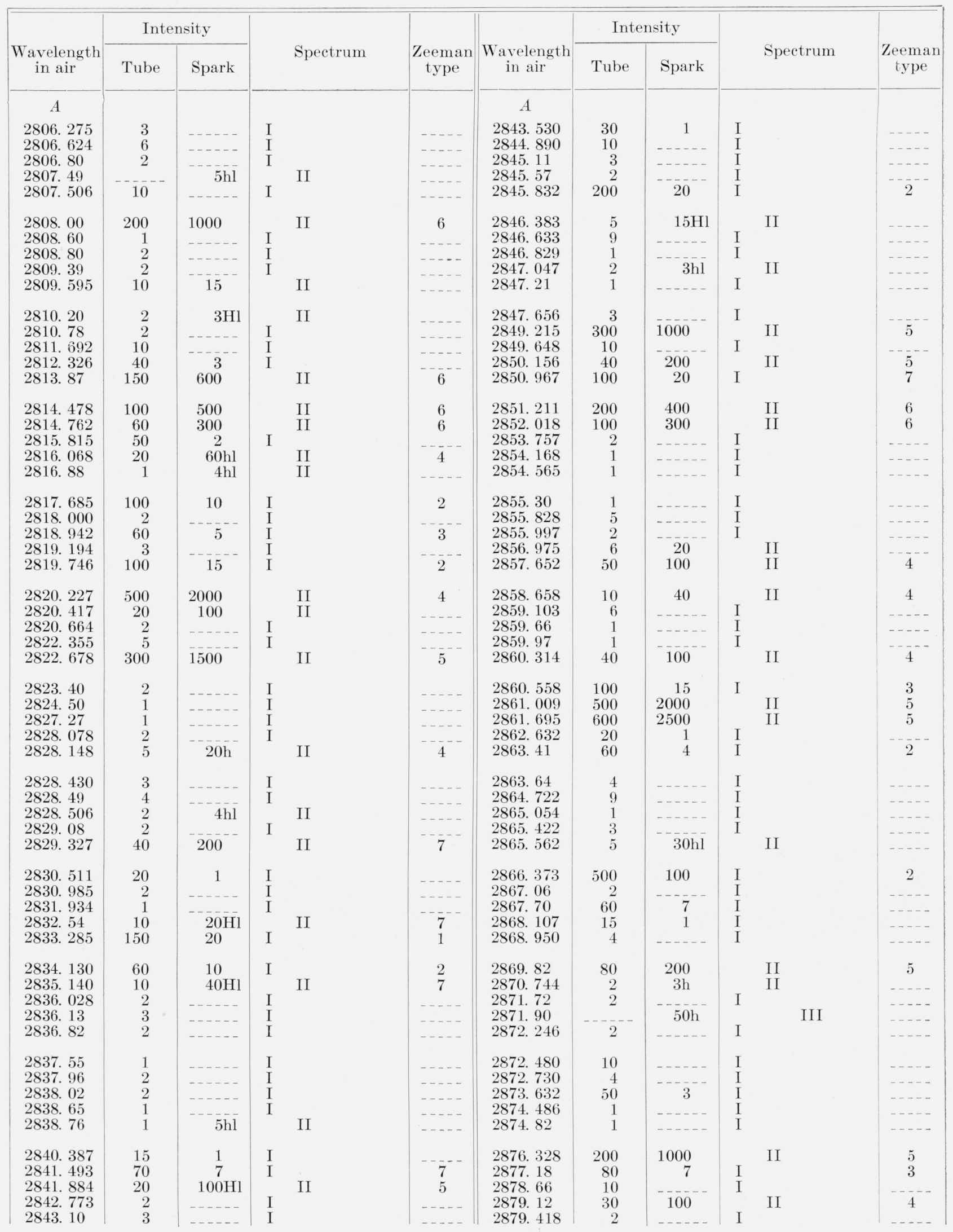


TABLE 1. Emission spectra of hafnium-Continued

\begin{tabular}{|c|c|c|c|c|c|c|c|c|c|c|}
\hline \multirow[b]{2}{*}{$\begin{array}{c}\text { Wavelength } \\
\text { in air }\end{array}$} & \multicolumn{2}{|c|}{ Intensity } & & \multirow[b]{2}{*}{ Spectrum } & \multirow[b]{2}{*}{$\begin{array}{c}\text { Zeeman } \\
\text { type }\end{array}$} & \multirow[b]{2}{*}{$\begin{array}{c}\text { Wavelength } \\
\text { in air }\end{array}$} & \multicolumn{2}{|c|}{ Intensity } & \multirow[b]{2}{*}{ Spectrum } & \multirow[b]{2}{*}{$\begin{array}{c}\text { Zeeman } \\
\text { type }\end{array}$} \\
\hline & Tube & Spark & & & & & Tube & Spark & & \\
\hline$A$ & & & & & & $A$ & & & & \\
\hline 2879. 72 & 10 & $30 \mathrm{Hl}$ & & II & -...- & 2918. 591 & 300 & 40 & I & 3 \\
\hline 2880.211 & 2 & $10 \mathrm{Hl}$ & & II & $-\cdots$ & 2919. 60 & 300 & 2000 & II & 6 \\
\hline 2880.84 & 1 & $\ldots \ldots$ & I & & $\ldots \ldots$ & 2922. 15 & 4 & $\ldots$ & I & \\
\hline 2882. 259 & 15 & - & I & & $\ldots$ & 2924. 613 & 150 & 20 & I & 7 \\
\hline 2882.57 & 1 & 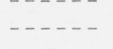 & I & & $\ldots$ & 2925. 965 & 10 & $-\ldots--$ & I & $\ldots$ \\
\hline 2883. 02 & 1 & _. - - - & I & & & 2926. 04 & 4 & 20 & II & $\ldots$ \\
\hline 2884. 12 & 1 & & $\mathrm{I}$ & & & 2926. 22 & 10 & $\ldots$ & I & $\ldots$ \\
\hline 2885. 474 & 40 & 200 & & II & 5 & 2926. 328 & 10 & 40 & II & 4 \\
\hline 2886. 91 & 10 & $10 \mathrm{hl}$ & & II & $\ldots$ & 2926. 58 & 7 & - - - - & I & - . - - \\
\hline 2887. 132 & 200 & 60 & I & & 1 & 2927.65 & 3 & - . & I & $\ldots$ \\
\hline 2887. 542 & 100 & 30 & I & & 2 & 2928. 980 & 40 & 6 & I & 1 \\
\hline 2888. 23 & 1 & $1 \mathrm{~h}$ & & II & $\ldots$ & 2929. 642 & 400 & 2000 & II & 7 \\
\hline 2888. 633 & 8 & $\ldots$ & I & & $\ldots$ & 2929. 895 & 200 & 50 & I & 1 \\
\hline 2889. 001 & 8 & 50 & & II & 4 & 2930.55 & 7 & $50 \mathrm{Hl}$ & II & 4 \\
\hline 2889.62 & 300 & 50 & I & & 1 & 2930. 69 & 4 & $\ldots \ldots$ & I & $\ldots$ \\
\hline 2891. 019 & 40 & 4 & I & & $\ldots$ & 2932. 92 & 1 & $2 \mathrm{~h}$ & II & $\ldots$ \\
\hline 2891. 46 & 1 & $\ldots$ & I & & $-\cdots$ & 2934. 641 & 20 & 1 & I & $-\cdots$ \\
\hline 2891. 89 & 2 & $\ldots \ldots$ & I & & $\ldots$ & 2935. 365 & 40 & 2 & I & 7 \\
\hline 2891. 954 & 3 & $--=-$ & I & & $\ldots$ & 2935. 725 & 10 & $\ldots \ldots$ & I & $\ldots$. \\
\hline 2892.285 & 4 & $5 \mathrm{hl}$ & & II & $\ldots$ & 2936.338 & 2 & $\ldots+\cdots$ & I & $\ldots$ \\
\hline 2892. 565 & 60 & 7 & I & & 7 & 2937. 782 & 400 & 3000 & II & 6 \\
\hline 2892. 98 & 3 & $\ldots \ldots$ & $\mathrm{I}$ & & $\ldots$ & 2938. 235 & 4 & $\ldots$ & I & $\ldots$ \\
\hline 2893. 03 & 2 & $\ldots$ & I & & $\ldots$ & 2938. 656 & 2 & $-\ldots--$ & I & $\ldots$. \\
\hline 2893. 751 & 10 & $\ldots \ldots$ & $\mathrm{I}$ & & $\ldots$ & 2940. 23 & 1 & $5 \mathrm{hl}$ & II & $\ldots \ldots$ \\
\hline 2894. 028 & 10 & $20 \mathrm{~h}$ & & II & 6 & 2940.762 & 800 & 100 & I & 3 \\
\hline 2894. 840 & 30 & 3 & I & & $-\ldots$ & 2942. 73 & 10 & $\ldots$ & I & $\ldots$ \\
\hline 2896. 260 & 10 & $\ldots-\ldots$ & I & & $\ldots$ & 2943. 70 & 2 & & I & $\ldots-\ldots$ \\
\hline 2897. 11 & 1 & & I & & $\ldots \ldots$ & 2944. 71 & 200 & 40 & $\vec{I}$ & 2 \\
\hline 2897. 34 & & $50 \mathrm{~h}$ & & III & -1 & 2947. 135 & 20 & 300 & II & 4 \\
\hline 2898. 256 & 500 & 50 & I & & 7 & 2947. 474 & 2 & $\ldots \ldots$ & I & $\ldots$ \\
\hline 2898. 702 & 50 & 200 & & II & 5 & 2947. 72 & 2 & & I & $\ldots$ \\
\hline 2900.12 & 1 & $\ldots \ldots$ & I & & $\ldots-\ldots$ & 2948. 390 & 6 & $\ldots$ & I & $\ldots$ \\
\hline 2900. 750 & 20 & $\ldots \ldots$ & I & & $\ldots$ & 2949.58 & 2 & $50 \mathrm{Hl}$ & II & $\ldots$. \\
\hline 2901. 16 & 4 & $\ldots-\ldots$ & I & & $\ldots$ & 2949. 81 & 5 & & I & - - \\
\hline 2901. 31 & 1 & $2 \mathrm{hl}$ & & II & $\ldots$ & 2950.67 & 600 & 100 & I & 3 \\
\hline 2901. 925 & 10 & & I & & & 2951. 900 & 20 & 5 & I & $\ldots-\ldots$ \\
\hline 2902. 641 & 30 & 1 & I & & & 2952.55 & 2 & $5 \mathrm{Hl}$ & II & $\ldots-$ \\
\hline 2902. 81 & 10 & & I & & $\ldots$ & 2953. 12 & 1 & $\ldots . .$. & I & $\ldots .$. \\
\hline 2904. 412 & 400 & 70 & $\mathrm{I}$ & & 3 & 2953. 36 & 1 & & $\mathrm{I}$ & $\ldots$. \\
\hline 2904.531 & 20 & $100 \mathrm{~h}$ & & II & $\ldots-$ & 2953. 647 & 3 & 30 & II & $\ldots \ldots$ \\
\hline 2904. 760 & 300 & 60 & I & & 1 & 2954. 201 & 500 & 100 & I & 1 \\
\hline 2905. 17 & 5 & $\ldots \ldots$ & $\mathrm{I}$ & & ..... & 2954.53 & 6 & & $\mathrm{I}$ & $\ldots$. \\
\hline 2906. 61 & 1 & $2 \mathrm{~h}$ & & II & $\ldots-\ldots$ & 2954. 935 & 2 & 7 & II & $\ldots$. \\
\hline 2907. 012 & 30 & 1 & I & & $\ldots$ & 2955. 64 & 4 & & I & $\ldots .$. \\
\hline 2907. 318 & 3 & $\ldots \ldots$ & I & & $\ldots-\ldots$ & 2955. 99 & 1 & $3 \mathrm{~h}$ & II & $\ldots$. \\
\hline 2907. 63 & 1 & $7 \mathrm{Hl}$ & & II & & 2956.376 & 30 & 1 & I & - \\
\hline 2908. 831 & 10 & $30 \mathrm{Hl}$ & & II & 5 & 2957.27 & 2 & $20 \mathrm{hl}$ & II & $\ldots .$. \\
\hline 2909. 912 & 200 & 300 & & II & 5 & 2958. 01 & 300 & 40 & I & 3 \\
\hline 2910. 46 & 1 & - - . - & I & & $\ldots$. & 2958.55 & 4 & & $\mathrm{I}$ & $\ldots-$ \\
\hline 2911. 836 & 2 & $\ldots \ldots$ & I & & $\ldots \ldots$ & 2959.144 & 2 & $20 \mathrm{Hl}$ & II & $\ldots-$ \\
\hline 2912. 760 & 10 & 20 & & II & 4 & 2960. 180 & 5 & & $\mathrm{I}$ & \\
\hline 2913. 179 & 10 & $50 \mathrm{hl}$ & & II & 4 & 2960.813 & 20 & 200 & II & 5 \\
\hline 2913. 56 & 5 & $\ldots \ldots$ & I & & $\ldots-\ldots$ & 2961. 80 & 40 & 400 & II & 6 \\
\hline 2913. 64 & 2 & ...... & I & & $\ldots \ldots$ & 2962. 592 & 1 & $\ldots \ldots$ & I & $\ldots \ldots$ \\
\hline 2915. 08 & 5 & $\ldots \ldots$ & I & & $\ldots$ & 2962. 921 & 2 & $\ldots \ldots$ & I & 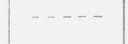 \\
\hline 2915. 27 & 5 & $\ldots \ldots$ & I & & $\ldots$ & 2963.547 & 5 & & I & $\ldots \ldots$ \\
\hline 2915. 72 & 1 & $\ldots \ldots$ & I & & $\ldots \ldots$ & 2963. 72 & 2 & $5 \mathrm{Hl}$ & II & $\ldots$ \\
\hline 2916. 48 & 800 & 100 & I & & 1 & 2963. 90 & 1 & $\ldots \ldots$ & I & $\ldots$. \\
\hline 2917. 308 & 8 & & $\mathrm{I}$ & & $\ldots-$ & 2963. 97 & 1 & & I & 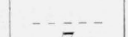 \\
\hline 2917. 487 & 20 & 200 & & II & 5 & 2964.885 & 400 & 80 & $\mathrm{I}$ & 7 \\
\hline
\end{tabular}


TABLE 1. Emission spectra of hafnium-Continued

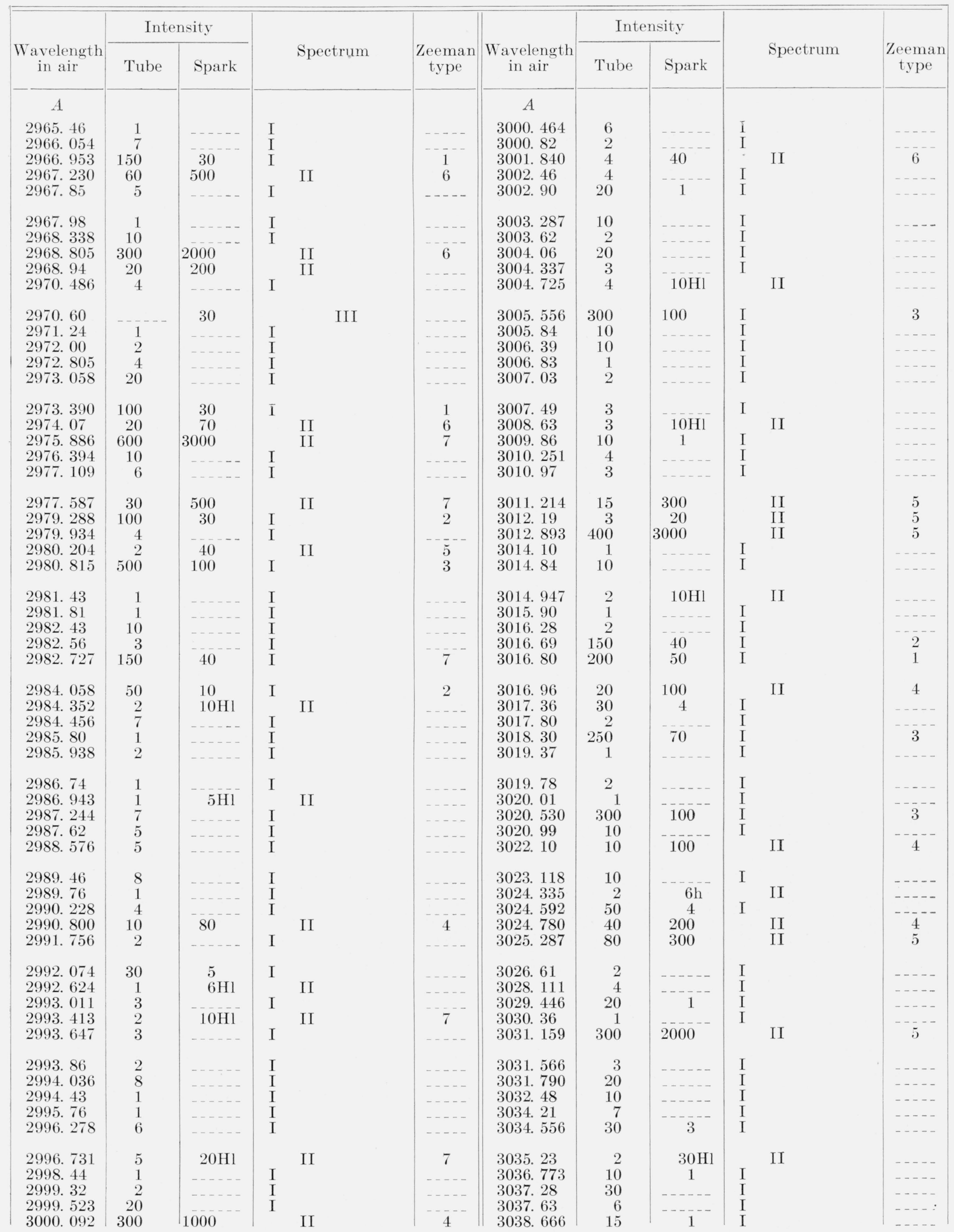


TABLE 1. Emission spectra of hafnium-Continued

\begin{tabular}{|c|c|c|c|c|c|c|c|c|c|c|c|}
\hline \multirow[b]{2}{*}{$\begin{array}{c}\text { Wavelength } \\
\text { in air }\end{array}$} & \multicolumn{2}{|c|}{ Intensity } & & \multirow[b]{2}{*}{ Spectrum } & \multirow[b]{2}{*}{$\begin{array}{c}\text { Zeeman } \\
\text { type }\end{array}$} & \multirow[b]{2}{*}{$\begin{array}{c}\text { Wavelength } \\
\text { in air }\end{array}$} & \multicolumn{2}{|c|}{ Intensity } & \multirow{2}{*}{\multicolumn{2}{|c|}{ Spectrum }} & \multirow[b]{2}{*}{$\begin{array}{c}\text { Zeeman } \\
\text { type }\end{array}$} \\
\hline & Tube & Spark & & & & & Tube & Spark & & & \\
\hline$A$ & & & & & & $A$ & & & & & \\
\hline 3039. 025 & 7 & - - & I & & $\ldots-\ldots$ & 3075.315 & 40 & 7 & I & & $\ldots-$ \\
\hline 3039. 647 & 15 & & $\mathrm{I}$ & & $\ldots$ & 3075. 874 & 20 & 1 & I & & \\
\hline 3039.70 & 3 & $10 \mathrm{Hl}$ & & II & $\ldots$ & 3076. 665 & 4 & 100 & & II & 6 \\
\hline 3041.004 & 2 & $\ldots$ & $\mathrm{I}$ & & $--1-$ & 3076. 885 & 30 & 1 & I & & $\ldots$. \\
\hline 3041.41 & 9 & $\ldots \ldots$ & I & & - . - & 3077. 76 & 2 & $\ldots \ldots$ & I & & $\cdots--$ \\
\hline 3042.085 & 30 & 4 & $\mathrm{I}$ & & 1 & 3078. 04 & 2 & & I & & $\ldots$ \\
\hline 3042.271 & 20 & $\ldots \ldots$ & I & & $\ldots$ & 3078. 77 & 6 & & I & & $\ldots .$. \\
\hline 3042.552 & 15 & $\ldots$ & I & & - . - & 3079. 10 & 2 & $3 \mathrm{Hl}$ & & II & $\ldots$. \\
\hline $\begin{array}{l}3042.654 \\
3043.30\end{array}$ & $\begin{array}{l}3 \\
3\end{array}$ & $15 \mathrm{Hl}$ & I & II & $\ldots-1$ & $\begin{array}{l}3080.27 \\
3080.635\end{array}$ & $\begin{array}{r}4 \\
100\end{array}$ & 2000 & I & II & 6 \\
\hline 3043.905 & 10 & & I & & $\ldots$ & 3080.842 & 200 & 50 & I & & 1 \\
\hline 3044. 103 & 20 & 1 & I & & $\ldots$ & 3081. 844 & 7 & $\ldots \ldots$ & I & & $\ldots$. \\
\hline 3045.28 & 1 & ----- & I & & -...- & 3082. 14 & 6 & $\ldots \ldots-$ & I & & .... \\
\hline 3045. 40 & 2 & $---\cdots-$ & I & & $\ldots$ & 3082. 526 & 7 & $\ldots \ldots$ & I & & $\ldots$ \\
\hline 3046. 030 & 40 & 400 & & II & 4 & 3083.085 & 8 & $\ldots \ldots-$ & I & & 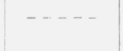 \\
\hline 3046.68 & 1 & $\ldots$ & I & & $\ldots \ldots$ & 3083. 151 & 15 & & I & & $\ldots$. \\
\hline 3047. 492 & 20 & 1 & I & & $\ldots$ & 3083. 53 & 3 & $30 \mathrm{Hl}$ & & II & $\ldots$. \\
\hline 3049.06 & 3 & & I & & $\ldots \ldots$ & 3083.628 & 30 & $\ldots \ldots$ & I & & $\ldots$. \\
\hline 3049.300 & 60 & 15 & I & & 3 & 3084. 743 & 20 & $\ldots \ldots$ & I & & $\ldots$. \\
\hline 3049.74 & 1 & $3 \mathrm{~h}$ & & II & $\ldots-$ & 3085. 17 & 2 & $\ldots \ldots$ & I & & $\cdots$ \\
\hline 3050.760 & 300 & 80 & I & & 2 & 3086. 580 & 2 & & I & & $\ldots \ldots$ \\
\hline 3051.16 & 5 & 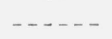 & I & & $\ldots$. & 3087. 196 & 60 & 6 & I & & $\ldots .$. \\
\hline 3051.30 & 10 & $\ldots-\ldots$ & I & & $\ldots$. & 3087. 947 & 8 & 1 & I & & $\ldots \ldots$ \\
\hline 3052. 75 & 1 & $\ldots \ldots$ & I & & $-\ldots-$ & 3088.533 & 60 & 5 & I & & $\ldots$ \\
\hline 3053.28 & 2 & 4 & & II & $\ldots$ & 3088. 985 & 4 & $\ldots \ldots$ & I & & $\ldots$ \\
\hline 3053. 74 & 1 & & I & & & 3089. 39 & 1 & & I & & $\ldots \ldots$ \\
\hline 3054.53 & 100 & 300 & & II & 6 & 3089.527 & 7 & $\ldots \ldots$ & I & & $\ldots$. \\
\hline 3055.415 & 30 & 400 & & II & 5 & 3090. 170 & 30 & 1 & I & & $\ldots$. \\
\hline 3056. 702 & 20 & 2 & I & & $\ldots \ldots$ & 3090. 516 & 20 & $-\ldots--$ & I & & $\ldots .$. \\
\hline 3057.010 & 400 & 100 & I & & 3 & 3091. 397 & 40 & 6 & I & & $\ldots-$ \\
\hline 3057.59 & 7 & $\ldots-\ldots$ & I & & $\ldots$ & 3091. 770 & 8 & $80 \mathrm{hl}$ & & II & 6 \\
\hline 3058.31 & 2 & - . - - & I & & $\ldots .$. & 3092. 250 & 60 & $500 \mathrm{hl}$ & & II & 5 \\
\hline 3058.61 & 3 & - . - - - & I & & $\ldots \ldots$ & 3093.65 & 1 & 2 & & II & -.... \\
\hline 3058. 66 & 1 & $1 \mathrm{~h}$ & & II & .... & 3095. 186 & $\begin{array}{r}4 \\
\end{array}$ & & I & & $-\cdots$ \\
\hline 3058.77 & 1 & $\ldots \ldots$ & I & & ---- & 3096. 764 & 150 & 40 & I & & 3 \\
\hline 3059. 343 & 10 & & $\mathrm{I}$ & & & 3097. 602 & 3 & & I & & $\ldots .$. \\
\hline 3060. 084 & $-\cdots$ & 100h & & III & ..... & 3098. 482 & 3 & -- & I & & $\cdots$ \\
\hline 3060.18 & 1 & $-\cdots$ & I & & $--\frac{--}{7}$ & $\begin{array}{l}3098.59 \\
3009\end{array}$ & 1 & - & I & & 5 \\
\hline $\begin{array}{l}3063.778 \\
3064.355\end{array}$ & $\begin{array}{r}80 \\
5\end{array}$ & $\begin{array}{l}10 \\
20\end{array}$ & I & II & $\begin{array}{c}7 \\
--\end{array}$ & $\begin{array}{l}3099.085 \\
3100.47\end{array}$ & $\begin{array}{l}2 \\
2\end{array}$ & $20 \mathrm{Hl}$ & I & II & $\ldots-$ \\
\hline 3064.686 & 40 & 500 & & II & 5 & 3100.792 & 30 & 1 & I & & \\
\hline 3066.18 & 30 & 1 & I & & $\ldots-\ldots$ & 3101.391 & 300 & 1000 & & II & 5 \\
\hline 3066.515 & 5 & $30 \mathrm{Hl}$ & & II & $\ldots$ & 3102. 391 & 8 & $\ldots \ldots$ & I & & $\ldots \ldots$ \\
\hline 3067. 426 & 200 & 40 & I & & 1 & 3103. 19 & 1 & & I & & $\ldots$ \\
\hline 3067. 614 & 60 & 10 & $\mathrm{I}$ & & $\ldots$. & 3103.410 & $\ldots-\ldots$ & $15 \mathrm{~h}$ & & III & $\ldots \ldots$ \\
\hline 3068.18 & 15 & 1 & $\mathrm{I}$ & & $\ldots \ldots$ & 3103. 66 & 20 & - & I & & $\ldots$. \\
\hline 3069.213 & 80 & 15 & I & & 1 & 3103. 712 & 50 & 1 & I & & $\ldots$ \\
\hline 3069. 645 & 2 & 10 & & II & $\ldots \ldots$ & 3104. 49 & 1 & $4 \mathrm{~h}$ & & II & $\ldots \ldots$ \\
\hline 3069. 726 & 7 & $\ldots \ldots$ & I & & $\ldots$. & 3104. 822 & 10 & $\ldots \ldots$ & I & & $\ldots \ldots$ \\
\hline 3069. 804 & 2 & $\ldots \ldots$ & $\mathrm{I}$ & & $\ldots \ldots$ & 3105.51 & 2 & $\ldots \ldots$ & I & & $\ldots \ldots$ \\
\hline 3069. 950 & 8 & $70 \mathrm{Hl}$ & & II & $\ldots-\ldots$ & 3106.01 & 2 & & $\mathrm{I}$ & & $\ldots \ldots$ \\
\hline 3070.095 & 20 & $\ldots-\ldots$ & I & & 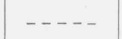 & 3106.14 & 1 & 1h & & II & $\ldots \ldots$ \\
\hline 3070.498 & 15 & $---\ldots$ & I & & $-\ldots--$ & 3106.43 & 1 & $\ldots \ldots$ & I & & $\ldots .$. \\
\hline 3070.85 & 1 & $\ldots \ldots$ & I & & $\ldots-$ & 3106.51 & 2 & - - & I & & $\ldots \ldots$ \\
\hline 3071.77 & 10 & - & 1 & & $\cdots--$ & 3106.75 & 1 & ----- & I & & $\ldots-$ \\
\hline 3072.365 & 20 & & $\mathrm{I}$ & & $\ldots-$ & 3106.93 & 1 & & $\mathrm{I}$ & & $\ldots \ldots$ \\
\hline 3072. 881 & 400 & 100 & I & & 2 & 3107. 815 & 3 & $7 \mathrm{Hl}$ & & II & $\ldots$. \\
\hline 3073.48 & 10 & $\ldots$ & I & & $\ldots \ldots$ & 3107.91 & 2 & $\ldots \ldots$ & I & & $\ldots \ldots$ \\
\hline 3074. 104 & 60 & 20 & I & & 3 & 3108. 043 & 10 & $\ldots \ldots$ & I & & $\ldots$ \\
\hline 3074.789 & 100 & 40 & I & & 2 & 3108.63 & 2 & $\ldots \ldots$ & 1 & & $\ldots \ldots$ \\
\hline
\end{tabular}


TABLE 1. Emission spectra of hafnium-Continued

\begin{tabular}{|c|c|c|c|c|c|c|c|c|c|c|}
\hline \multirow{2}{*}{$\begin{array}{l}\text { Wavelength } \\
\text { in air }\end{array}$} & \multicolumn{2}{|c|}{ Intensity } & \multirow{2}{*}{\multicolumn{2}{|c|}{ Spectrum }} & \multirow{2}{*}{$\begin{array}{c}\text { Zeeman } \\
\text { type }\end{array}$} & \multirow{2}{*}{$\begin{array}{l}\text { Wavelength } \\
\text { in air }\end{array}$} & \multicolumn{2}{|c|}{ Intensity } & \multirow{2}{*}{ Spectrum } & \multirow{2}{*}{$\begin{array}{c}\text { Zeeman } \\
\text { type }\end{array}$} \\
\hline & Tube & Spark & & & & & Tube & Spark & & \\
\hline \begin{tabular}{l}
\multicolumn{1}{c}{$A$} \\
3109.121 \\
3109.852 \\
3110.882 \\
3111.472 \\
3111.99
\end{tabular} & $\begin{array}{r}400 \\
4 \\
50 \\
4 \\
1\end{array}$ & $\begin{array}{r}2000 \\
400 \\
20\end{array}$ & $\begin{array}{l}\mathrm{I} \\
\mathrm{I}\end{array}$ & $\begin{array}{l}\text { II } \\
\text { II } \\
\text { II }\end{array}$ & $\begin{array}{c}6 \\
-1 \\
-\cdots\end{array}$ & \begin{tabular}{l}
\multicolumn{1}{c}{$A$} \\
3147.22 \\
3147.28 \\
3148.10 \\
3148.415 \\
3148.898
\end{tabular} & $\begin{array}{r}2 \\
4 \\
3 \\
100 \\
5\end{array}$ & 40 & $\begin{array}{l}\text { I } \\
\text { I } \\
\text { I } \\
\text { I } \\
\text { I }\end{array}$ & 1 \\
\hline $\begin{array}{l}3112.43 \\
3112.82 \\
3113.447 \\
3113.68 \\
3114.059\end{array}$ & $\begin{array}{l}1 \\
1 \\
7 \\
2 \\
1\end{array}$ & $\begin{array}{r}2 \mathrm{hl} \\
-3 \mathrm{hl} \\
-\end{array}$ & $\begin{array}{l}\mathrm{I} \\
\mathrm{I} \\
\mathrm{I}\end{array}$ & $\begin{array}{l}\text { II } \\
\text { II }\end{array}$ & $\begin{array}{l}---- \\
---- \\
---- \\
---- \\
----\end{array}$ & $\begin{array}{l}3149.064 \\
3149.824 \\
3150.332 \\
3151.64 \\
3151.962\end{array}$ & $\begin{array}{r}20 \\
15 \\
15 \\
80 \\
8\end{array}$ & $\begin{array}{r}2 \\
1 \\
1 \\
20\end{array}$ & $\begin{array}{l}\text { I } \\
\text { I } \\
\text { I } \\
\text { I } \\
\text { I }\end{array}$ & $2^{-}$ \\
\hline $\begin{array}{l}3114.56 \\
3114.896 \\
3116.19 \\
3116.506 \\
3116.947\end{array}$ & $\begin{array}{r}2 \\
15 \\
1 \\
30 \\
20\end{array}$ & 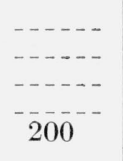 & $\begin{array}{l}\text { I } \\
\text { I } \\
\text { I } \\
\text { I }\end{array}$ & II & $\frac{}{-\cdots}$ & $\begin{array}{l}3152.59 \\
3152.841 \\
3152.952 \\
3153.52 \\
3154.64\end{array}$ & $\begin{array}{r}20 \\
7 \\
30 \\
3 \\
2\end{array}$ & $\begin{array}{c}2 \\
6 \\
-\cdots\end{array}$ & $\begin{array}{l}\mathrm{I} \\
\mathrm{I} \\
\mathrm{I} \\
\mathrm{I} \\
\mathrm{I}\end{array}$ & $\begin{array}{l}\cdots- \\
\cdots \cdots \\
\cdots- \\
\cdots-\end{array}$ \\
\hline $\begin{array}{l}3118.33 \\
3118.63 \\
3118.77 \\
3119.24 \\
3119.31\end{array}$ & $\begin{array}{r}10 \\
2 \\
4 \\
1 \\
2\end{array}$ & 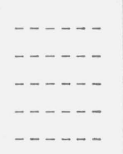 & $\begin{array}{l}\text { I } \\
\text { I } \\
\text { I } \\
\text { I } \\
\text { I }\end{array}$ & & $\begin{array}{l}--- \\
-\cdots \\
-\cdots- \\
-\cdots \\
\cdots--\end{array}$ & $\begin{array}{l}3156.48 \\
3156.688 \\
3157.08 \\
3158.44 \\
3158.71\end{array}$ & $\begin{array}{r}20 \\
200 \\
3 \\
1 \\
4\end{array}$ & $\begin{array}{c}2 \\
100 \\
-\ldots \\
-\end{array}$ & $\begin{array}{l}\text { I } \\
\text { I } \\
\text { I } \\
\text { I } \\
\text { I }\end{array}$ & ${ }^{--}$ \\
\hline $\begin{array}{l}3119.980 \\
3120.645 \\
3122.09 \\
3122.322 \\
3122.55\end{array}$ & $\begin{array}{r}100 \\
15 \\
1 \\
7 \\
2\end{array}$ & $\begin{array}{c}40 \\
1 \\
6 \mathrm{Hl} \\
- \\
-\end{array}$ & $\begin{array}{l}\mathrm{I} \\
\mathrm{I} \\
\mathrm{I} \\
\mathrm{I}\end{array}$ & II & \begin{tabular}{c}
7 \\
$-\cdots$ \\
\hdashline$-\cdots$ \\
\hdashline---
\end{tabular} & $\begin{array}{l}3158.82 \\
3159.84 \\
3159.96 \\
3160.231 \\
3161.09\end{array}$ & $\begin{array}{r}7 \\
300 \\
10 \\
10 \\
2\end{array}$ & $\begin{array}{c}40 \\
50 \\
-\end{array}$ & $\begin{array}{l}\mathrm{I} \\
\mathrm{I} \\
\mathrm{I}\end{array}$ & ${ }^{--}$ \\
\hline $\begin{array}{l}3122.87 \\
3122.948 \\
3123.51 \\
3123.68 \\
3123.973\end{array}$ & $\begin{array}{r}10 \\
1 \\
1 \\
1\end{array}$ & $\begin{array}{c}7 \mathrm{~h} \\
-\cdots-- \\
-----\end{array}$ & $\begin{array}{l}\mathrm{I} \\
\mathrm{I} \\
\mathrm{I} \\
\mathrm{I}\end{array}$ & II & 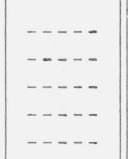 & $\begin{array}{l}3161.23 \\
3161.536 \\
3162.575 \\
3162.624 \\
3163.360\end{array}$ & $\begin{array}{r}1 \\
20 \\
300 \\
100 \\
30\end{array}$ & $\begin{array}{r}3 \\
30 \\
500 \\
3\end{array}$ & $\begin{array}{ll}\text { I } & \\
\text { I } & \\
\text { I } & \\
\text { I } & \text { II }\end{array}$ & $\begin{array}{l}3 \\
3 \\
7 \\
-\end{array}$ \\
\hline $\begin{array}{l}3124.510 \\
3124.89 \\
3126.278 \\
3126.655 \\
3126.95\end{array}$ & $\begin{array}{r}20 \\
1 \\
20 \\
20 \\
5\end{array}$ & $\begin{array}{c}1 \\
100 \\
4 \\
----\end{array}$ & $\begin{array}{l}\mathrm{I} \\
\mathrm{I} \\
\mathrm{I} \\
\mathrm{I}\end{array}$ & II & 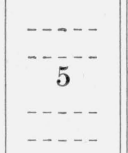 & $\begin{array}{l}3163.90 \\
3164.385 \\
3165.27 \\
3165.730 \\
3166.97\end{array}$ & $\begin{array}{r}3 \\
100 \\
1 \\
50 \\
3\end{array}$ & $\begin{array}{c}5 \mathrm{hl} \\
20^{10} \\
-\end{array}$ & $\begin{array}{l}\text { I } \\
\text { I } \\
\text { I } \\
\text { I }\end{array}$ & $7^{-}$ \\
\hline $\begin{array}{l}3127.390 \\
3128.760 \\
3129.588 \\
3130.40 \\
3130.60\end{array}$ & $\begin{array}{r}10 \\
100 \\
80 \\
3 \\
1\end{array}$ & $\begin{array}{c}20 \\
15 \\
2 \mathrm{hl}\end{array}$ & $\begin{array}{l}\text { I } \\
\text { I } \\
\text { I } \\
\text { I }\end{array}$ & II & $\begin{array}{l}---1 \\
-\cdots \\
-\cdots- \\
-\cdots- \\
-\cdots\end{array}$ & $\begin{array}{l}3167.15 \\
3167.454 \\
3168.26 \\
3168.390 \\
3168.957\end{array}$ & $\begin{array}{r}1 \\
10 \\
8 \\
100 \\
2\end{array}$ & $\begin{array}{c}30 \\
40 \\
3 \mathrm{hl}\end{array}$ & $\begin{array}{ll}\text { I } & \\
& \text { II } \\
\text { I } & \\
\text { I } & \\
& \text { II }\end{array}$ & 7 \\
\hline $\begin{array}{l}3131.812 \\
3132.236 \\
3133.03 \\
3133.078 \\
3133.30\end{array}$ & $\begin{array}{r}400 \\
20 \\
1 \\
3 \\
1\end{array}$ & $\begin{array}{r}200 \\
3 \\
60\end{array}$ & $\begin{array}{l}\mathrm{I} \\
\mathrm{I} \\
\mathrm{I} \\
\mathrm{I}\end{array}$ & II & $\begin{array}{c}7 \\
\cdots- \\
\cdots-- \\
\cdots--\end{array}$ & $\begin{array}{l}3169.50 \\
3169.60 \\
3169.762 \\
3170.83 \\
3172.08\end{array}$ & $\begin{array}{r}4 \\
10 \\
2 \\
1\end{array}$ & $\begin{array}{r}7 \mathrm{hl} \\
\ldots \ldots \\
-\ldots-\end{array}$ & $\begin{array}{l}\text { I } \\
\text { I } \\
\text { I } \\
\text { I }\end{array}$ & 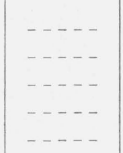 \\
\hline $\begin{array}{l}3133.83 \\
3134.725 \\
3135.562 \\
3135.770 \\
3137.520\end{array}$ & $\begin{array}{r}1 \\
600 \\
30 \\
20 \\
70\end{array}$ & $\begin{array}{r}5000 \\
2 \\
1 \\
30\end{array}$ & $\begin{array}{l}\mathrm{I} \\
\mathrm{I} \\
\mathrm{I} \\
\mathrm{I}\end{array}$ & II & 6 & $\begin{array}{l}3172.24 \\
3172.949 \\
3173.65 \\
3174.882 \\
3175.957\end{array}$ & $\begin{array}{r}6 \\
300 \\
1 \\
30 \\
10\end{array}$ & $\begin{array}{c}70 \\
4 \mathrm{hl} \\
6\end{array}$ & $\begin{array}{l}\text { I } \\
\text { I } \\
\text { I } \\
\text { I }\end{array}$ & 3 \\
\hline $\begin{array}{l}3138.43 \\
3138.651 \\
3139.29 \\
3139.663 \\
3140.771\end{array}$ & $\begin{array}{r}20 \\
200 \\
4 \\
100 \\
40\end{array}$ & $\begin{array}{c}1 \\
20 \\
-500 \\
500\end{array}$ & $\begin{array}{l}\text { I } \\
\text { I } \\
\text { I }\end{array}$ & $\begin{array}{l}\text { II } \\
\text { II }\end{array}$ & $\frac{5}{7}$ & $\begin{array}{l}3176.858 \\
3178.05 \\
3178.436 \\
3179.62 \\
3180.19\end{array}$ & $\begin{array}{r}200 \\
10 \\
40 \\
40 \\
10\end{array}$ & $\begin{array}{c}600 \\
5 \\
5\end{array}$ & $\begin{array}{l}\text { I } \\
\text { I } \\
\text { I } \\
\text { I }\end{array}$ & $\begin{array}{c}4 \\
1 \\
-1\end{array}$ \\
\hline $\begin{array}{l}3142.57 \\
3144.46 \\
3145.315 \\
3146.22 \\
3146.95\end{array}$ & $\begin{array}{r}1 \\
7 \\
200 \\
2 \\
-\end{array}$ & $\frac{500}{40 h}$ & $\begin{array}{l}\mathrm{I} \\
\mathrm{I} \\
\mathrm{I}\end{array}$ & II & $\begin{array}{c}-\ldots \\
\cdots \\
\cdots-n\end{array}$ & $\begin{array}{l}3180.81 \\
3181.008 \\
3181.148 \\
3181.756 \\
3182.57\end{array}$ & $\begin{array}{r}20 \\
80 \\
70 \\
5 \\
2\end{array}$ & $\begin{array}{r}2 \\
10 \\
10 \\
50 \\
\end{array}$ & $\begin{array}{ll}\text { I } & \\
\text { I } & \\
\text { I } & \\
\text { I } & \text { II }\end{array}$ & $\begin{array}{l}7 \\
1 \\
5\end{array}$ \\
\hline
\end{tabular}


TABLE 1. Emission spectra of hafnium-Continued

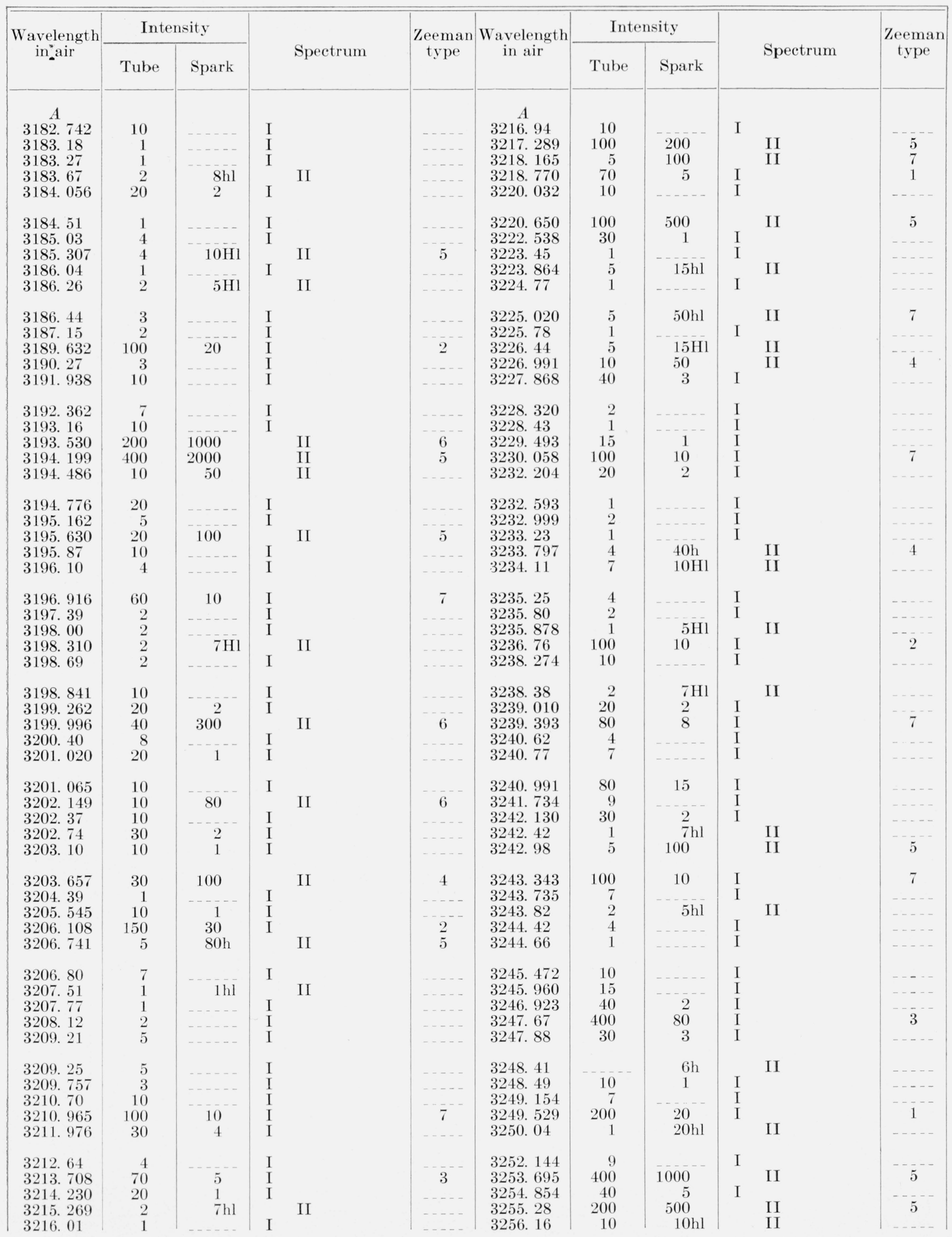


TABLE 1. Emission spectra of hafnium-Continued

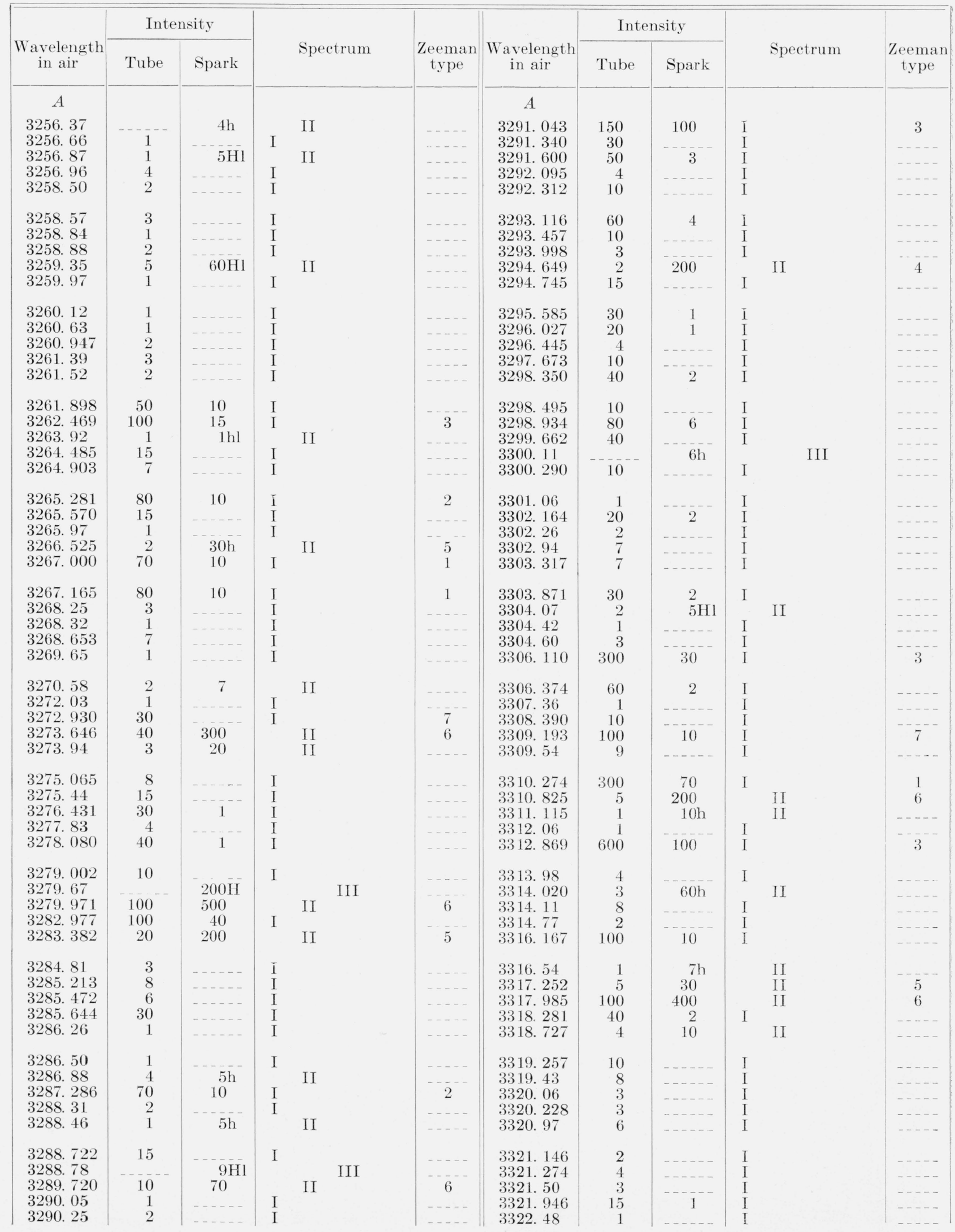


TABLE 1. Emission spectra of hafnium-Continued

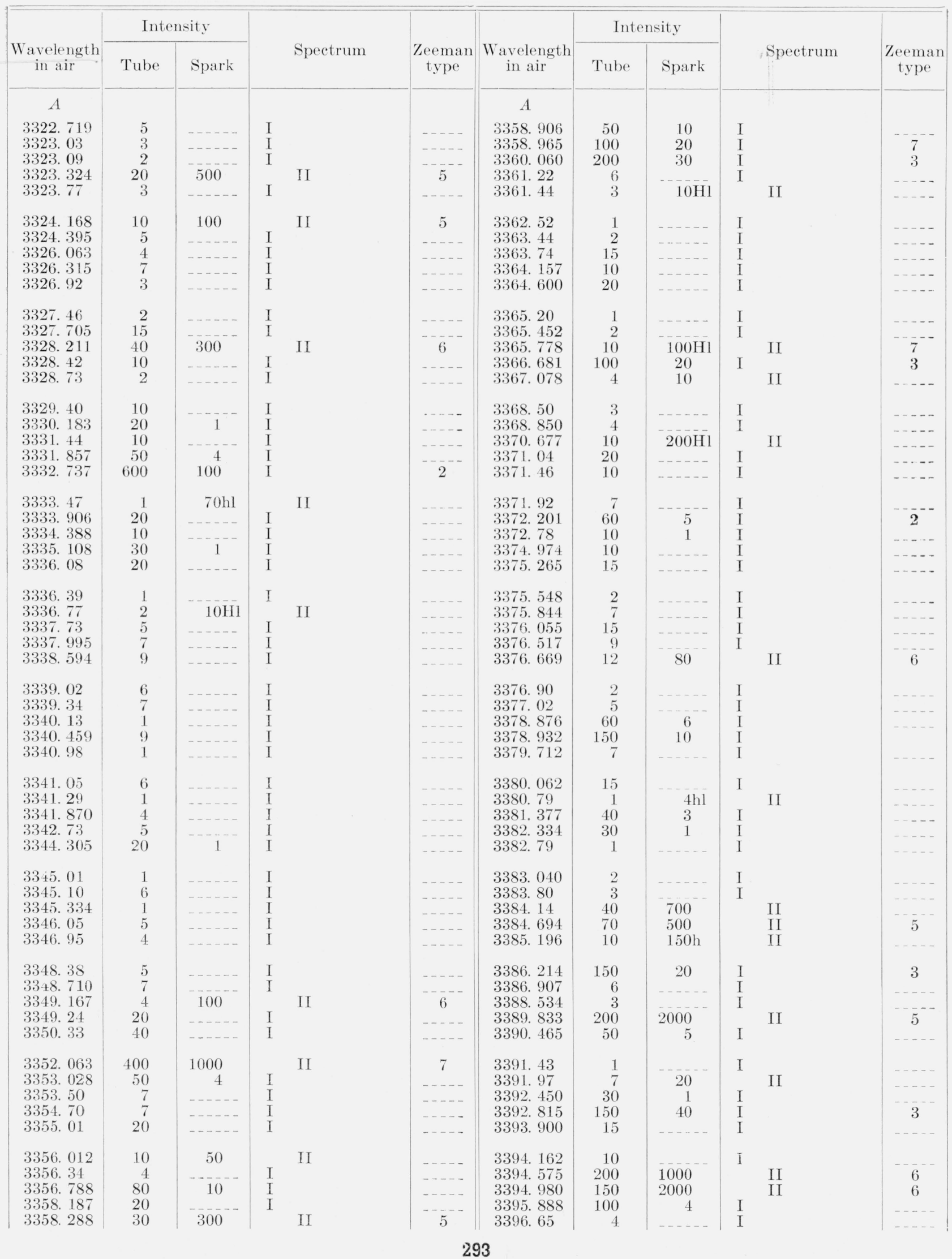


TABLE 1. Emission spectra of hafnium-Continued

\begin{tabular}{|c|c|c|c|c|c|c|c|c|c|c|}
\hline \multirow[b]{2}{*}{$\begin{array}{c}\text { Wavelength } \\
\text { in air }\end{array}$} & \multicolumn{2}{|c|}{ Intensity } & \multirow{2}{*}{\multicolumn{2}{|c|}{ Spectrum }} & \multirow[b]{2}{*}{$\begin{array}{c}\text { Zeeman } \\
\text { type }\end{array}$} & \multirow[b]{2}{*}{$\begin{array}{c}\text { Wavelength } \\
\text { in air }\end{array}$} & \multicolumn{2}{|c|}{ Intensity } & \multirow[b]{2}{*}{ Spectrum } & \multirow[b]{2}{*}{$\begin{array}{c}\text { Zeeman } \\
\text { type }\end{array}$} \\
\hline & Tube & Spark & & & & & Tube & Spark & & \\
\hline$A$ & & & & & & $A$ & & & & \\
\hline 3397.257 & 200 & 20 & I & & 3 & 3440.860 & 100 & 6 & I & \\
\hline 3397. 602 & 100 & 20 & I & & 7 & 3441. 835 & 150 & 8 & $\mathrm{I}$ & 2 \\
\hline 3398.54 & 3 & & I & & & 3443. 412 & 2 & $10 \mathrm{~h}$ & II & \\
\hline 3399. 793 & 1000 & 5000 & & II & 5 & 3444.140 & 7 & $\ldots \ldots$ & I & $\ldots$ \\
\hline 3400.218 & 200 & 20 & I & & $\ldots$ & 3445. 068 & 10 & $\ldots$ & I & $\cdots$ \\
\hline 3401.73 & 3 & 2 & I & & & 3446. 481 & 10 & $10 \mathrm{~h}$ & II & \\
\hline 3402.512 & 200 & 20 & I & & 2 & 3447.446 & 30 & 2 & I & - \\
\hline 3405. 304 & 6 & $\ldots$ & I & & $\ldots$ & 3448. 284 & 100 & 8 & $\mathrm{I}$ & 7 \\
\hline 3405. 89 & 4 & $\ldots \ldots$ & I & & $\ldots$ & 3448. 969 & 50 & 3 & I & $\ldots$ \\
\hline 3407.140 & 50 & 10 & I & & 3 & 3449.82 & 6 & $\ldots \ldots$ & $\vec{I}$ & $\ldots$ \\
\hline 3407.750 & 40 & 700 & & II & 5 & 3450.33 & 2 & $10 \mathrm{Hl}$ & II & $\ldots \ldots$ \\
\hline 3407.928 & 20 & $\ldots \ldots$ & I & & $\ldots \ldots$ & 3450.54 & 1 & $\ldots \ldots$ & I & 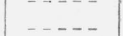 \\
\hline 3408. 63 & 7 & $\ldots$ & $\mathrm{I}$ & & $\ldots$ & 3451.522 & 20 & 1 & $\mathrm{I}$ & $\ldots$ \\
\hline 3408. 679 & 8 & $\ldots \ldots$ & I & & $-\ldots$ & 3451. 64 & 1 & $7 \mathrm{Hl}$ & II & $\ldots$ \\
\hline 3409.546 & 20 & 1 & $\mathrm{I}$ & & $\ldots$. & 3452. 304 & 70 & 10 & I & 2 \\
\hline 3409. 724 & 10 & & I & & & 3452.576 & 15 & & I & - - - \\
\hline 3410.157 & 150 & 2000 & & II & 6 & 3452.619 & 10 & $\ldots \ldots$ & $\mathrm{I}$ & $-\ldots$ \\
\hline 3410.892 & 7 & $\ldots \ldots$ & I & & $\ldots \ldots$ & 3453. 136 & 40 & 3 & $\mathrm{I}$ & $-\ldots$ \\
\hline 3411. 253 & 10 & $\ldots \ldots$ & I & & - & 3453.525 & 40 & 3 & $\mathrm{I}$ & $\ldots$ \\
\hline 3411.734 & 5 & $\ldots$ & I & & $-\ldots$ & 3455.858 & 10 & $\ldots \ldots$ & $\mathrm{I}$ & - \\
\hline 3412.016 & 20 & & I & & & 3455. 998 & 10 & 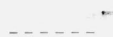 & $\mathrm{I}$ & \\
\hline 3412.374 & 100 & 10 & I & & 2 & 3456. 39 & 2 & $\ldots \ldots$ & $\mathrm{I}$ & 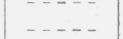 \\
\hline 3412.687 & 8 & & $\mathrm{I}$ & & & 3456.831 & 30 & $\ldots \ldots$ & $\mathrm{I}$ & $-\ldots$ \\
\hline 3413. 732 & 30 & $400 \mathrm{~h}$ & & II & 5 & 3457.559 & 1 & $1 \mathrm{~h}$ & II & $-\ldots$ \\
\hline 3414.313 & 7 & $\ldots \ldots$ & I & & $\ldots$. & 3458.18 & 1 & $\ldots \ldots$ & I & $\ldots$ \\
\hline 3414.543 & 2 & -. & I & & $\ldots$ & 3458.69 & 8 & $\ldots \ldots$ & I & $\therefore \ldots$ \\
\hline 3415. 20 & 2 & $9 \mathrm{Hl}$ & & II & $\ldots \ldots$ & 3459.98 & 2 & $\ldots \ldots$ & I & $\ldots \ldots$ \\
\hline 3416.04 & 3 & $15 \mathrm{Hl}$ & & II & $\ldots$ & 3460.720 & 20 & $\ldots \ldots$ & $\mathrm{I}$ & $\ldots$ \\
\hline 3416.52 & 1 & $\ldots \ldots$ & I & the & $-\ldots$ & 3461. 250 & 6 & $\ldots \ldots$ & $\mathrm{I}$ & $\ldots$ \\
\hline 3416.82 & 3 & $\ldots .$. & I & & $\ldots$. & 3461.33 & 5 & $\ldots \ldots$ & I & $\ldots$. \\
\hline 3417.34 & 300 & 30 & $I$ & & 2 & 3461.77 & 3 & & I & \\
\hline 3419. 171 & 500 & 90 & $\mathrm{I}$ & & 1 & 3462. 12 & 5 & & $\mathrm{I}$ & \\
\hline 3419.80 & 5 & $\ldots \ldots$ & $\mathrm{I}$ & & $\ldots$ & 3462. 646 & 150 & 300 & II & 5 \\
\hline 3419.87 & 2 & $\ldots . .$. & I & & $\ldots$. & 3464. 12 & $\underset{-2}{2}$ & & I & $\ldots$ \\
\hline 3420.00 & 1 & $\ldots$ & I & & $\ldots$ & 3464.720 & 50 & 2 & I & - \\
\hline 3420.775 & 20 & & I & & & 3465. 120 & 30 & 1 & $\bar{I}$ & \\
\hline 3421.435 & 40 & 100 & & II & 5 & 3465. 93 & 5 & 40 & II & 6 \\
\hline 3422.542 & 15 & $\ldots \ldots$ & I & & $\ldots \ldots$ & 3467. 410 & 20 & 1 & I & $\ldots \ldots$ \\
\hline 3423. 08 & 7 & $\ldots \ldots$ & $\mathrm{I}$ & & $\ldots \ldots$ & 3467.569 & 150 & 15 & $\mathrm{I}$ & 2 \\
\hline 3423. 500 & 20 & $\ldots \ldots$ & $\bar{I}$ & & -. & 3468. 810 & 3 & 15 & II & $\ldots$. \\
\hline 3423. 656 & 8 & - & I & & - & 3468. 99 & 5 & & I & - \\
\hline 3426.478 & 30 & 1 & I & & & 3469. 260 & 5 & 20 & II & $\ldots$ \\
\hline 3427.430 & 150 & 20 & $\mathrm{I}$ & & 1 & 3469.91 & 1 & $\ldots \ldots$ & I & $\ldots$ \\
\hline 3428.363 & 200 & 500 & & II & 5 & 3470.300 & 7 & $\ldots \ldots$ & $\vec{I}$ & $\ldots \ldots$ \\
\hline 3431. 189 & 10 & $\ldots .$. & I & & $\ldots$. & 3470.800 & 15 & $\ldots \ldots$ & I & $\ldots$ \\
\hline 3431. 79 & 3 & $\ldots \ldots$ & I & & $\ldots$ & 3470.910 & 10 & & I & \\
\hline 3432.28 & 2 & $\ldots \ldots$ & I & & $\ldots$ & 3472.410 & 800 & 100 & I & 1 \\
\hline 3432.577 & 9 & $\ldots \ldots$ & I & & & 3473.982 & 30 & $\ldots \ldots$ & I & $-1-\ldots$ \\
\hline 3434. 639 & 50 & 4 & I & & $\ldots \ldots$ & 3475.16 & 60 & 3 & $\mathrm{I}$ & $-\ldots$ \\
\hline 3434. 969 & 15 & 1 & $\mathrm{I}$ & & $\ldots$ & 3475.483 & 30 & 2 & $\mathrm{I}$ & $\ldots$ \\
\hline 3435. 277 & 15 & & I & & & 3475.59 & 6 & $20 \mathrm{Hl}$ & II & \\
\hline 3435.58 & 1 & $8 \mathrm{hl}$ & & II & $\ldots \ldots$ & 3476.450 & 20 & $\ldots \ldots$ & I & $\ldots \ldots$ \\
\hline 3436. 438 & 40 & 2 & I & & $\ldots \ldots$ & 3476.573 & 40 & $\ldots \ldots$ & $\mathrm{I}$ & $\ldots \ldots$ \\
\hline 3436.81 & 9 & $\ldots \ldots$ & $\mathrm{I}$ & & $\ldots \ldots$ & 3477.96 & 2 & $10 \mathrm{hl}$ & II & $\ldots$ \\
\hline 3437.15 & 1 &.- & I & & $\ldots$ & 3478.978 & 100 & 1000 & II & 6 \\
\hline 3437.30 & $\ldots$ & $10 \mathrm{H}$ & & III & & 3479. 284 & 250 & 1500 & II & 7 \\
\hline 3438. 29 & & $5 \mathrm{H}$ & & III & & 3481.05 & 7 & $\ldots \ldots$ & I & $\ldots \ldots$ \\
\hline 3438.428 & 300 & 30 & I & & 7 & 3481. 410 & 15 & $\ldots \ldots$ & $\mathrm{I}$ & $\ldots \ldots$ \\
\hline 3439.669 & 20 & 1 & $\mathrm{I}$ & & & 3481.895 & 70 & 3 & I & $\ldots \ldots$ \\
\hline 3440.07 & 1 & $10 \mathrm{Hl}$ & & II & & 3483.111 & 50 & 2 & I & $\ldots$ \\
\hline
\end{tabular}


TABLE 1. Emission spectra of hafnium-Continued

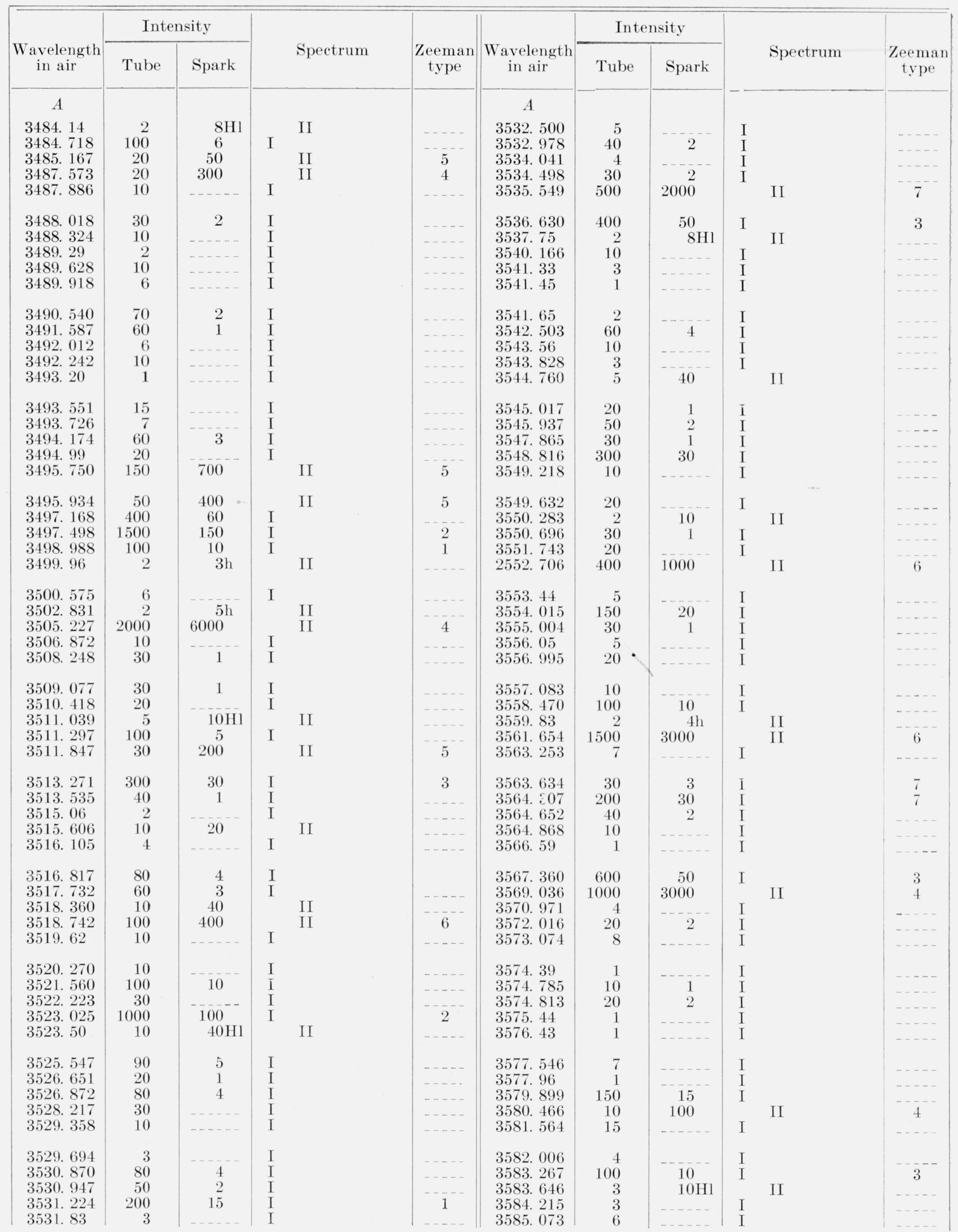


TABLE 1. Emission spectra of hafnium-Continued

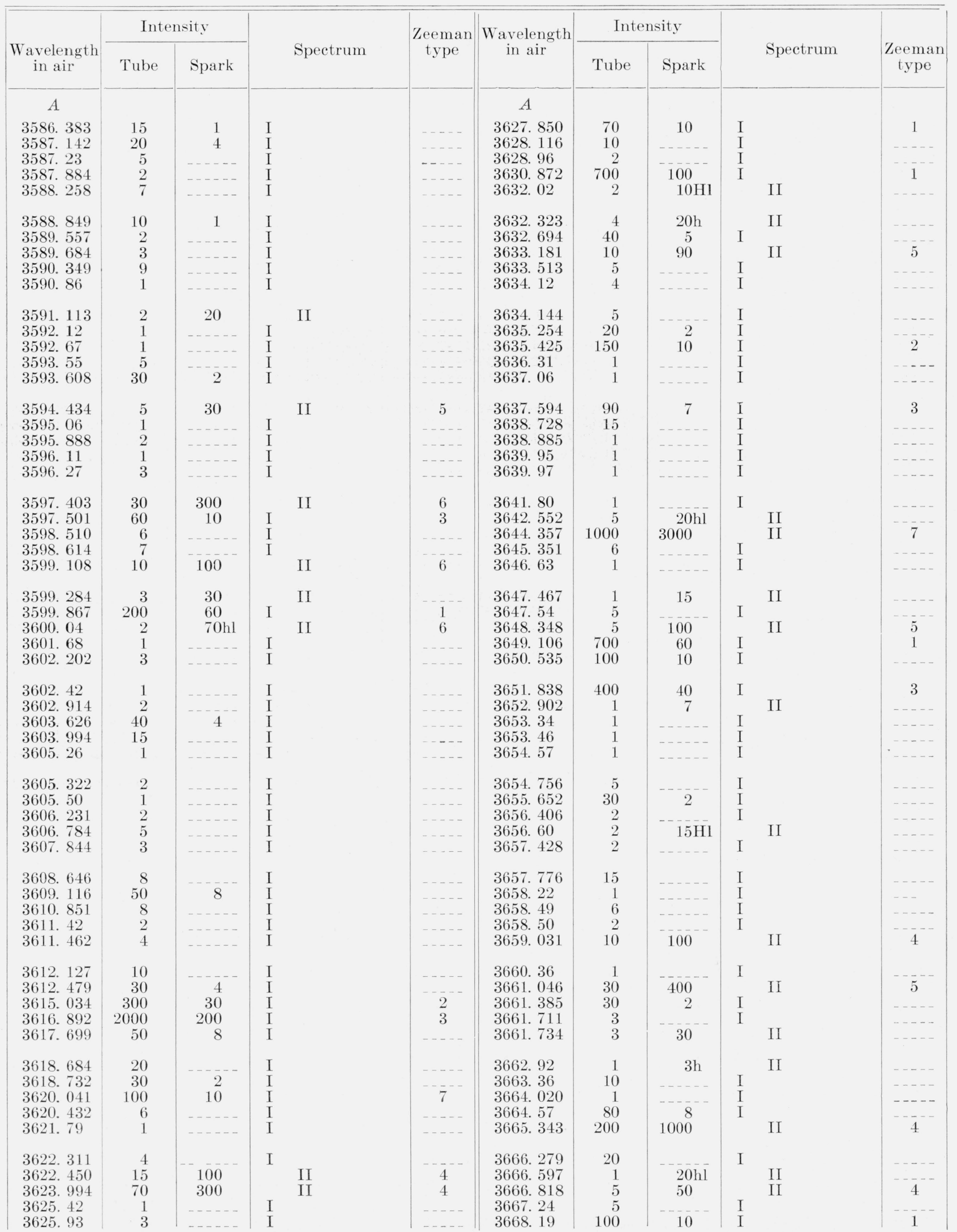


TABLE 1. Emission spectra of hafnium-Continued

\begin{tabular}{|c|c|c|c|c|c|c|c|c|c|c|}
\hline \multirow{2}{*}{$\begin{array}{l}\text { Wavelength } \\
\text { in air }\end{array}$} & \multicolumn{2}{|c|}{ Intensity } & \multirow{2}{*}{ Spectrum } & \multirow{2}{*}{$\begin{array}{c}\text { Zeeman } \\
\text { type }\end{array}$} & \multirow{2}{*}{$\begin{array}{c}\text { Wavelength } \\
\text { in air }\end{array}$} & \multicolumn{2}{|c|}{ Intensity } & \multirow{2}{*}{\multicolumn{2}{|c|}{ Spectrum }} & \multirow{2}{*}{$\begin{array}{c}\text { Zeeman } \\
\text { type }\end{array}$} \\
\hline & Tube & Spark & & & & Tube & Spark & & & \\
\hline$A$ & & & & & $A$ & & & & & \\
\hline 3668.59 & 2 & & $\mathrm{I}$ & $\ldots \ldots$ & 3716. 054 & 30 & 2 & I & & - . . - \\
\hline 3669.49 & 15 & 1 & $\mathrm{I}$ & & 3717.144 & 80 & $\overline{7}$ & I & & \\
\hline 3672.13 & 1 & $10 \mathrm{Hl}$ & II & $\ldots-\ldots$ & 3717.802 & 1000 & 100 & $\mathrm{I}$ & & 7 \\
\hline 3672. 304 & 300 & 30 & I & 7 & 3718. 781 & 2 & 30 & & II & \\
\hline 3673.06 & 3 & $\ldots \ldots$ & I & $\ldots$. & 3719.278 & 300 & 3000 & & II & 4 \\
\hline 3674.07 & 2 & & I & & 3721.500 & 80 & 7 & I & & \\
\hline 3675. 742 & 400 & 150 & I & 1 & 3722.66 & 1 & 20 & & II & 6 \\
\hline 3676.34 & 9 & $\ldots \ldots$ & $\mathrm{I}$ & $\ldots$ & 3722.904 & 4 & $-\ldots-\ldots$ & I & & $\ldots \ldots$ \\
\hline 3676. 716 & 3 & $\ldots \ldots$ & I & ..... & 3723.38 & 5 & $\ldots \ldots$ & I & & $\ldots \ldots$ \\
\hline 3677.50 & 1 & $-\ldots \ldots$ & I & $\ldots-$. & 3724.444 & 2 & $\ldots \ldots$ & I & & $\ldots \ldots$ \\
\hline 3677.681 & 3 & & I & $\ldots \ldots$ & 3725.68 & 1 & & I & & $\ldots \ldots$ \\
\hline 3678.028 & 2 & $60 \mathrm{hl}$ & II & 5 & 3726.485 & 150 & 15 & I & & 2 \\
\hline 3681. 384 & 20 & 100 & II & 4 & 3727.092 & 3 & $\ldots-\ldots$ & I & & $\ldots-\ldots$ \\
\hline 3682. 247 & 3000 & 200 & I & 3 & 3728. 179 & 2 & $-\ldots$ & I & & $\ldots$ \\
\hline 3683.57 & 5 & $\ldots \ldots$ & $\mathrm{I}$ & $\ldots$. & 3728.378 & 3 & $\ldots-\ldots$ & I & & $\ldots \ldots$ \\
\hline 3685. 352 & 3 & $\ldots \ldots$ & I & $\ldots \ldots$ & 3729. 093 & 150 & 15 & I & & 7 \\
\hline 3685.94 & 4 & $\ldots \ldots$ & I & $\ldots$ & 3730.760 & 20 & 1 & I & & $\ldots \ldots$ \\
\hline 3687.42 & 9 & $\ldots \ldots$ & I & $\ldots$ & 3732.99 & 2 & $\ldots \ldots$ & I & & $\ldots \ldots$ \\
\hline 3687.76 & 2 & $\ldots \ldots$ & I & $\ldots-\ldots$ & 3733. 786 & 1000 & 100 & I & & 1 \\
\hline 3687.84 & 2 & $\ldots \ldots$ & I & $\ldots$ & 3735.364 & 5 & $\ldots \ldots$ & I & & $\ldots \ldots$ \\
\hline 3688. 756 & 9 & & I & & 3736. 303 & 20 & 1 & I & & \\
\hline 3690.10 & 3 & -. & I & & 3737. 866 & 30 & 1000 & & II & 7 \\
\hline 3691. 185 & 20 & 2 & I & $\ldots-\ldots$ & 3739. 030 & 150 & 10 & I & & 3 \\
\hline 3691. 45 & 2 & $\ldots \ldots$ & I & $\ldots$ & 3739. 68 & 2 & $\ldots-\ldots$ & I & & $\ldots \ldots$ \\
\hline 3692.770 & 4 & -.... & I & $\ldots$ & 3739.835 & 2 & $7 \mathrm{Hl}$ & & II & 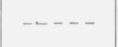 \\
\hline 3693. 156 & 10 & $\ldots$ & I & $\ldots$ & 3741.12 & 2 & $\ldots$ & I & & $\ldots \ldots$ \\
\hline 3693.60 & 20 & 3 & I & $\ldots \ldots$ & 3741.396 & 6 & & I & & $\ldots$ \\
\hline 3694. 16 & 40 & 4 & I & $\ldots \ldots$ & 3741.94 & & $100 \mathrm{H}$ & & III & $\ldots \ldots$ \\
\hline 3695. 72 & 2 & & I & $\ldots \ldots$ & 3744.002 & 30 & 2 & I & & $\ldots$ \\
\hline 3696.524 & 400 & 40 & I & 1 & 3744. 958 & 20 & 500 & & II & 6 \\
\hline 3697.08 & 50 & 4 & I & $\ldots$ & 3745. 765 & 2 & $7 \mathrm{hl}$ & & II & \\
\hline 3697.61 & 4 & & $\mathrm{I}$ & & 3746.810 & 400 & 60 & I & & 1 \\
\hline 3698. 392 & 30 & 400 & II & 4 & 3747.481 & 20 & 200 & & II & 7 \\
\hline 3698. 83 & 3 & $\ldots$ & 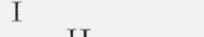 & $\ldots$ & 3748.554 & 5 & $\ldots \ldots$ & I & & $\ldots \ldots$ \\
\hline 3699. 55 & $\ldots \ldots$ & 10 & II & - - - & 3748.672 & 3 & $\ldots \ldots-$ & I & & $\ldots-\cdots$ \\
\hline 3699.57 & 5 & & I & & 3748. 71 & 1 & $\ldots \ldots$ & I & & $\ldots \ldots$ \\
\hline 3699. 73 & 70 & 1000 & II & 7 & 3749.829 & 8 & $\ldots$ & I & & $\ldots \ldots$ \\
\hline 3701.158 & 80 & 2000 & II & 4 & 3750.55 & 1 & $\ldots \ldots$ & I & & $\ldots \ldots$ \\
\hline 3701.83 & 15 & $\ldots \ldots--$ & I & $--\overline{--}$ & 3751. 778 & 10 & $\ldots \ldots$ & I & & $\ldots \ldots$ \\
\hline 3702.31 & 3 & $60 \mathrm{hl}$ & II & 7 & 3751.824 & 15 & 1 & I & & 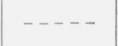 \\
\hline 3702.93 & 1 & $\ldots \ldots$ & I & $\ldots \ldots$ & 3752.784 & 20 & 2 & I & & \\
\hline 3703. 154 & 2 & - . - . & I & $\ldots \ldots$ & 3753. 225 & 100 & 20 & I & & 3 \\
\hline 3703. 816 & 10 & -.... & I & $\ldots \ldots$ & 3754. 26 & 1 & $\ldots \ldots$ & I & & $\ldots$. \\
\hline 3704. 067 & 15 & $\ldots \ldots$ & I & $\ldots \ldots$ & 3755. 024 & 5 & $\ldots \ldots$ & I & & $\ldots \ldots$ \\
\hline 3704.351 & 6 & $\ldots \ldots$ & $\mathrm{I}$ & & 3755.620 & 4 & $\ldots \ldots$ & I & & $\ldots$. \\
\hline 3704.657 & 2 & $30 \mathrm{hl}$ & II & $\ldots$ & 3755.71 & 2 & - . . - & I & & $\ldots \ldots$ \\
\hline 3704.938 & 40 & 3 & $I$ & $\ldots$ & 3755.99 & 1 & $\ldots \ldots$ & $\mathrm{I}$ & & $\ldots \ldots$ \\
\hline 3705.404 & 20 & 500 & II & 6 & 3756.17 & 1 & $\ldots \ldots$ & I & & $\ldots \ldots$ \\
\hline 3707. 02 & 1 & $-\cdots--$ & I & --- & 3756. 645 & 2 & $\ldots \ldots$ & I & & $\ldots \ldots$ \\
\hline 3707.374 & 70 & 7 & I & 1 & 3756.850 & 3 & $\ldots-\cdots$ & I & & $-\cdots-$ \\
\hline 3707. 92 & 1 & - . . . & I & $\ldots \ldots$ & 3756.98 & 1 & & I & & \\
\hline 3708.500 & 3 & 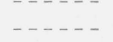 & I & $-1-1$ & 3757.554 & 20 & 1 & $\mathrm{I}$ & & $-\ldots$ \\
\hline 3708.85 & 1 & $\ldots \ldots$ & I & $\ldots$. & 3758.020 & 7 & 100 & & II & 4 \\
\hline 3709.044 & 4 & $-\cdots=-1$ & $\mathrm{I}$ & $\ldots-\ldots$ & 3760. 75 & 1 & $\ldots--$ & I & & $\ldots-\cdots$ \\
\hline 3711.142 & 4 & $50 \mathrm{hl}$ & II & $\ldots-$ & 3761.79 & 2 & $\ldots \ldots$ & I & & $\ldots \ldots$ \\
\hline 3711.692 & 3 & - - - - & I & $\ldots$ & 3762.500 & 30 & 1000 & & II & 4 \\
\hline 3711.83 & 1 & $-\ldots--$ & I & $\ldots-$ & 3763. 232 & 20 & 2 & I & & 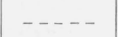 \\
\hline 3712. 22 & 4 & ---- & I & $\ldots \ldots$ & 3763.524 & 1 & $\ldots \ldots$ & I & & $\ldots \ldots$ \\
\hline 3712.52 & 1 & 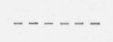 & I & $\ldots \ldots$ & 3763. 943 & 4 & ------ & I & & $--\overline{7--}$ \\
\hline 3712.642 & 7 & 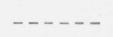 & $\mathrm{I}$ & $\ldots \ldots$ & 3764.528 & 90 & 8 & I & & 7 \\
\hline
\end{tabular}


TABLE 1. Emission spectra of hafnium-Continued

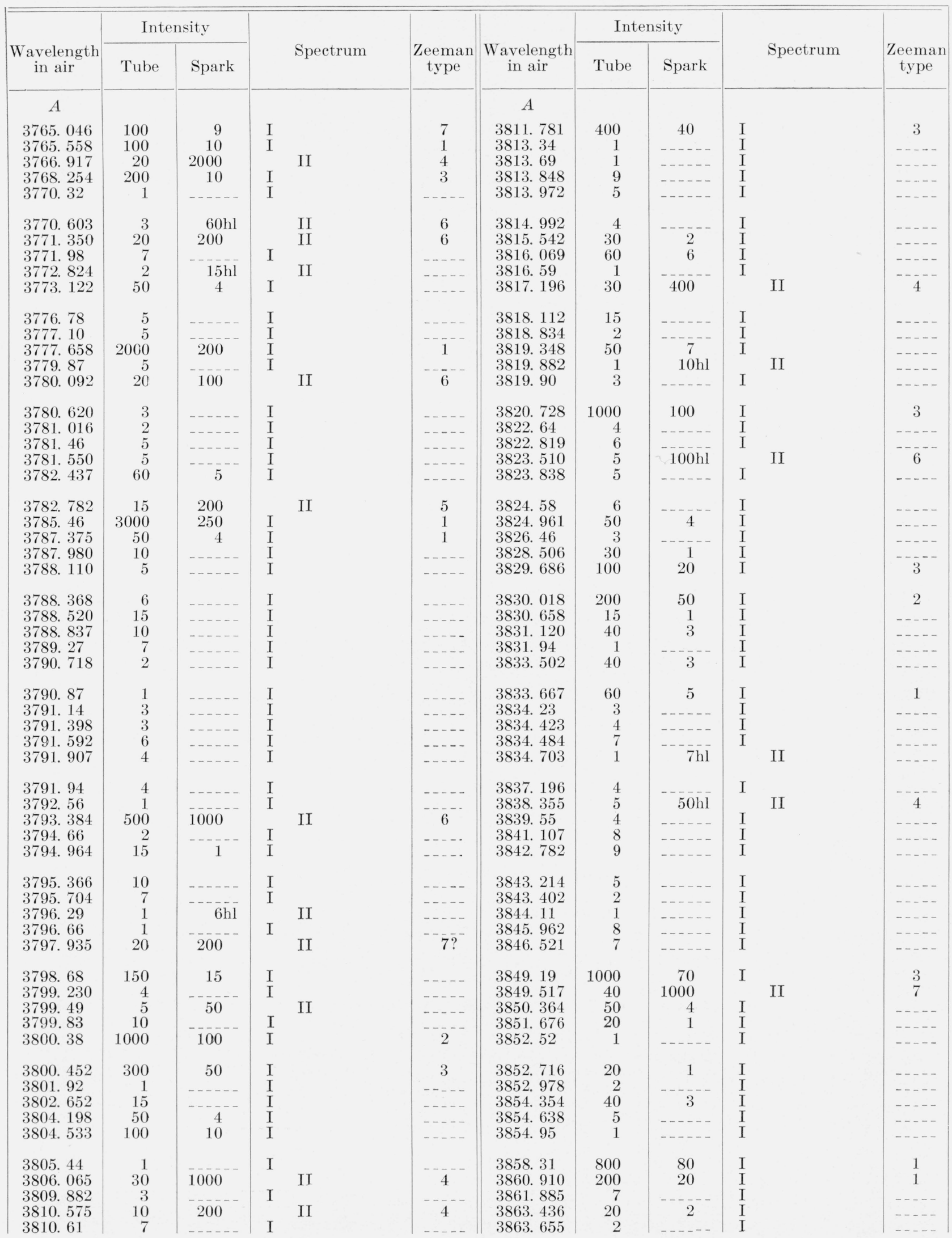


TABLE 1. Emission spectra of hafnium-Continued

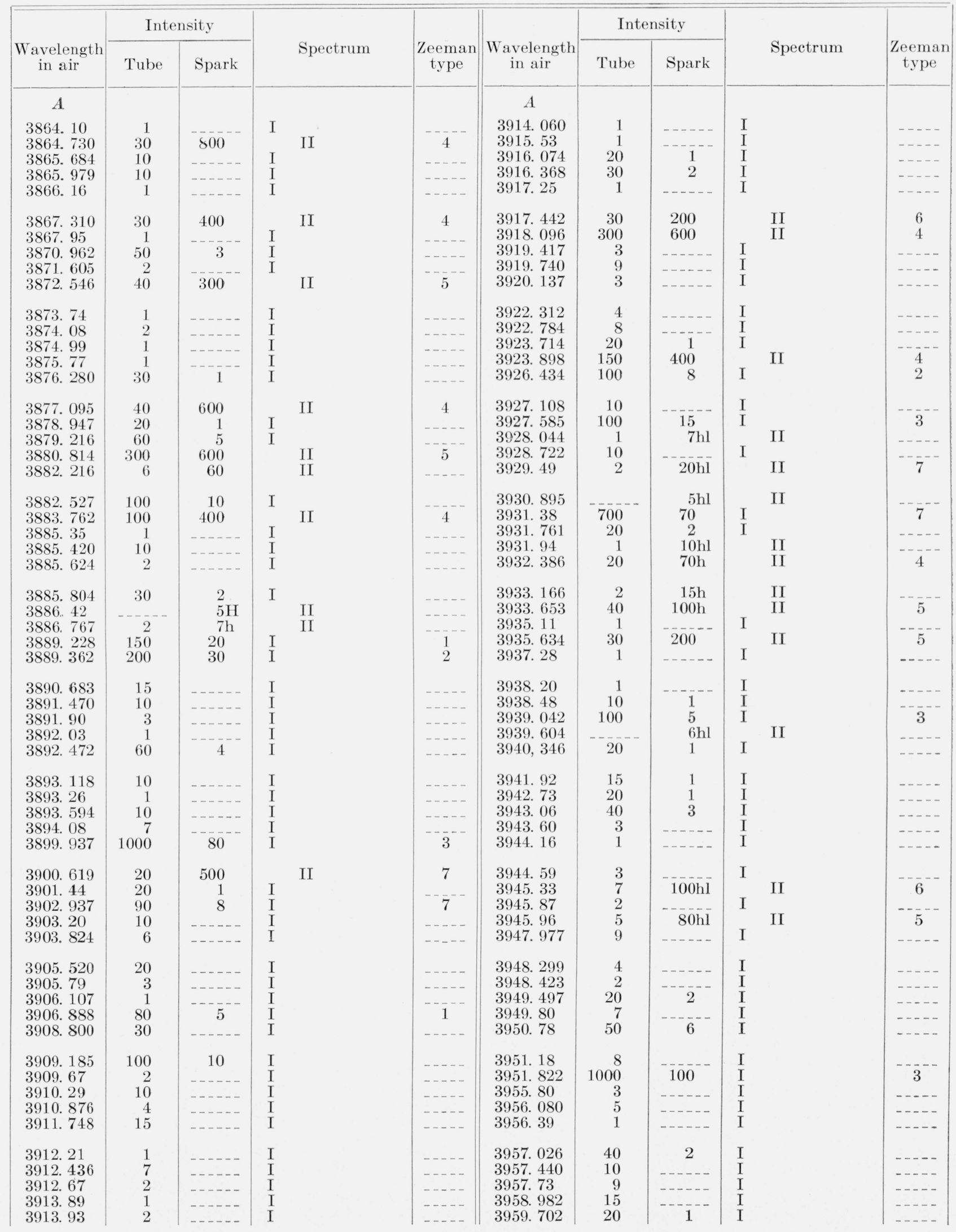


TABLE 1. Emission spectra of hafnium-Continued

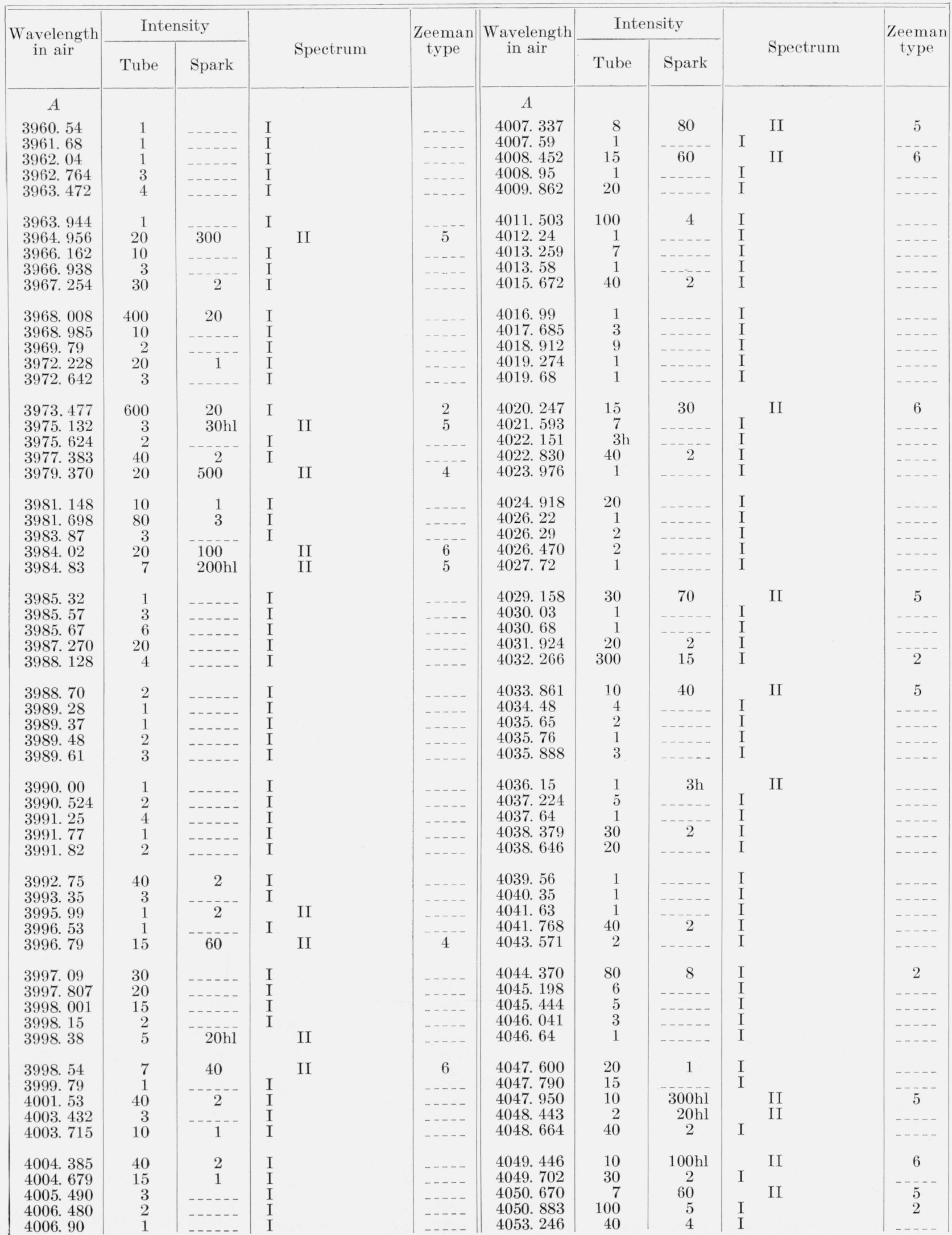


TABLE 1. Emission spectra of hafnium-Continued

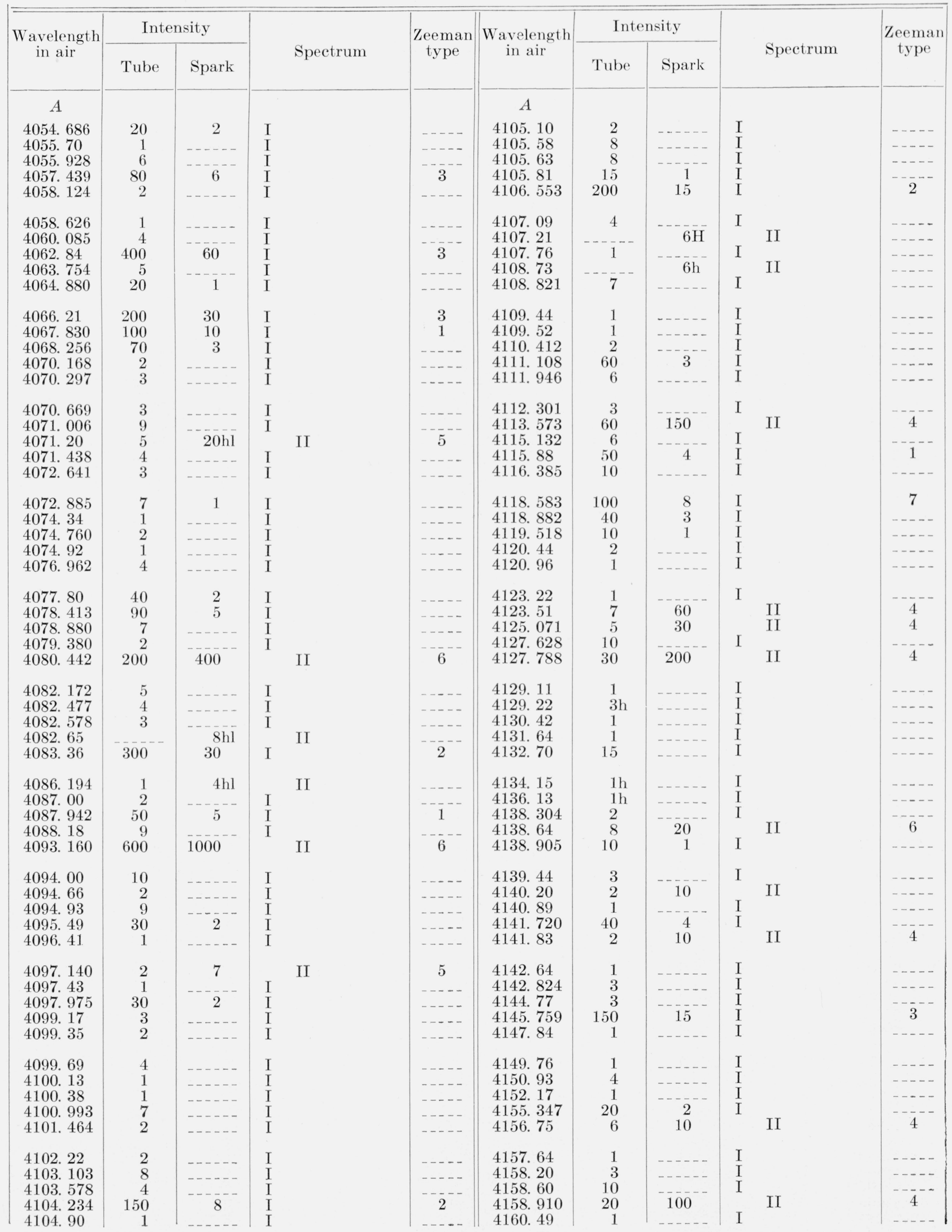


TABLE 1. Emission spectra of hafnium-Continued

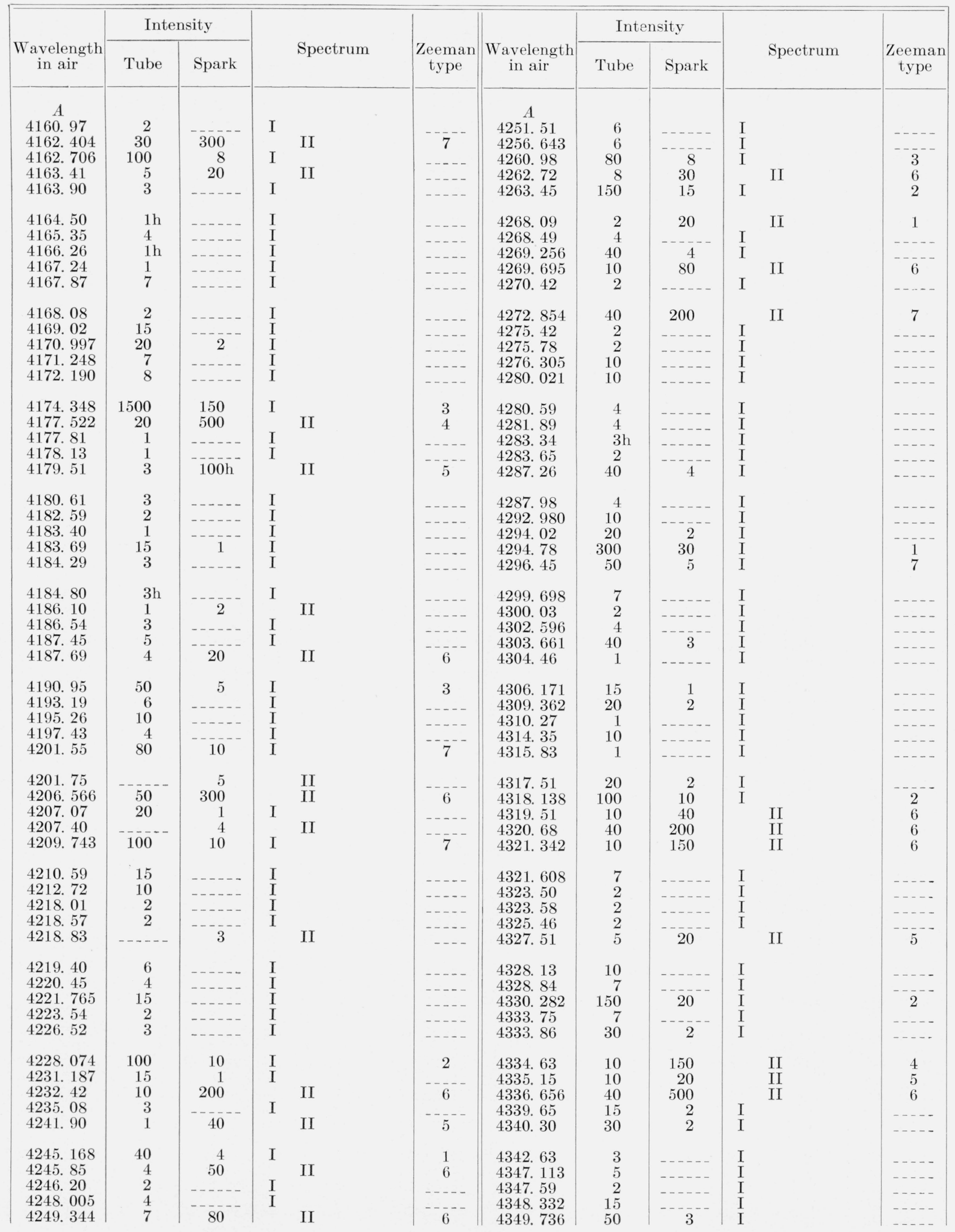


TABLE 1. Emission spectra of hafnium-Continued

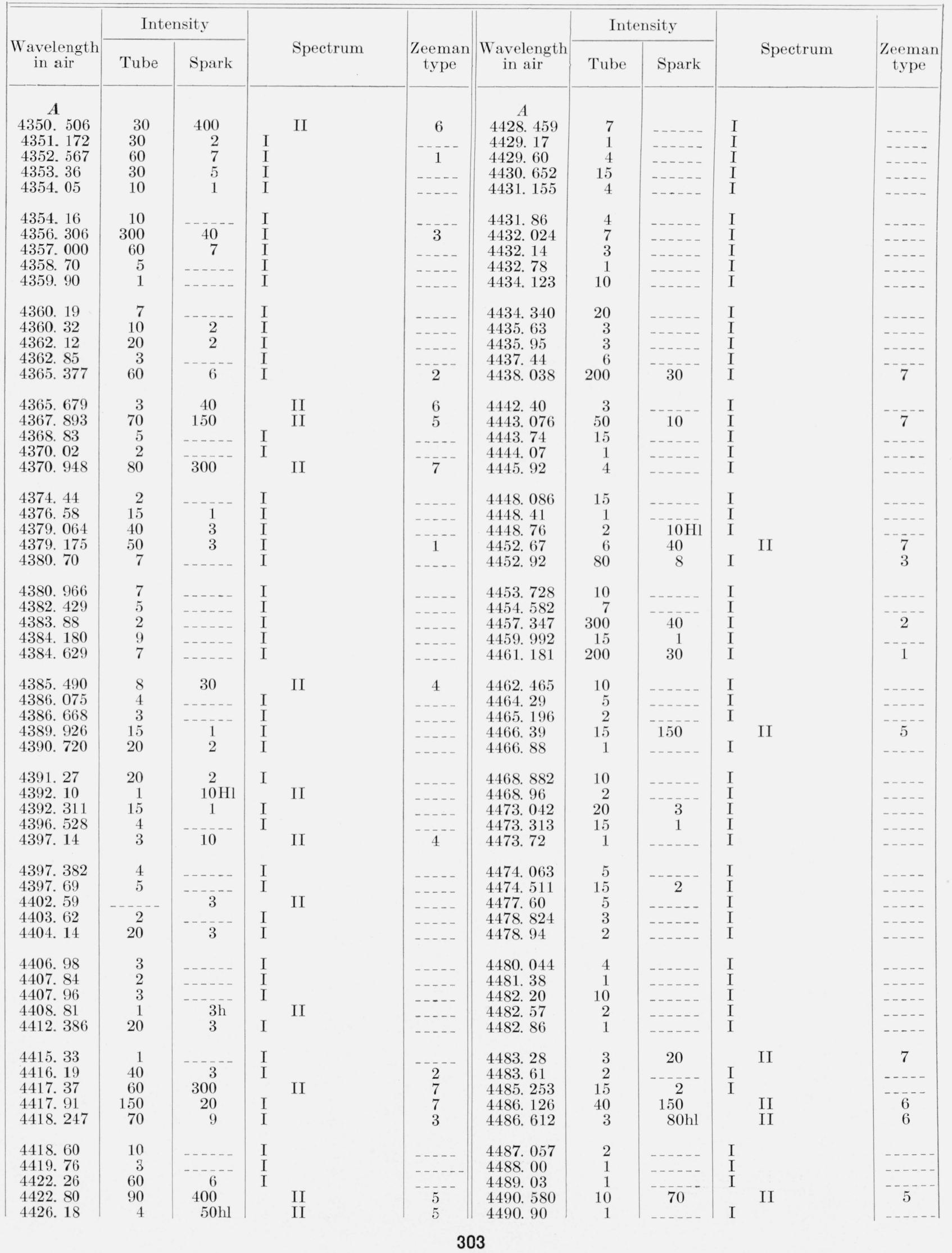


TABLE 1. Emission spectra of hafnium-Continued

\begin{tabular}{|c|c|c|c|c|c|c|c|c|c|}
\hline \multirow{2}{*}{$\begin{array}{c}\text { Wavelength } \\
\text { in air }\end{array}$} & \multicolumn{2}{|c|}{ Intensity } & \multirow{2}{*}{ Spectrum } & \multirow{2}{*}{$\begin{array}{c}\text { Zeeman } \\
\text { type }\end{array}$} & \multirow{2}{*}{$\begin{array}{c}\text { Wavelength } \\
\text { in air }\end{array}$} & \multicolumn{2}{|c|}{ Intensity } & \multirow{2}{*}{ Spectrum } & \multirow{2}{*}{$\begin{array}{c}\text { Zeeman } \\
\text { type }\end{array}$} \\
\hline & Tube & Spark & & & & Tube & Spark & & \\
\hline$A$ & & & & & $A$ & & & & \\
\hline 4491. 37 & 1 & $\ldots \ldots$ & $\mathrm{I}$ & & 4550.16 & 40 & 3 & I & 7 \\
\hline 4492. 16 & 30 & 2 & I & 2 & 4550.576 & 8 & 1 & $\mathrm{I}$ & \\
\hline 4493. 23 & 2 & ----- & I & $\ldots \ldots$ & 4553. 776 & 80 & 8 & I & 7 \\
\hline $\begin{array}{l}\text { 4494. } 235 \\
4495.08\end{array}$ & $\begin{array}{l}3 \\
1\end{array}$ & $-\cdots$ & $\begin{array}{l}\text { I } \\
\text { I }\end{array}$ & $-\cdots-$ & $\begin{array}{l}\text { 4556. } 85 \\
455976\end{array}$ & $\begin{array}{r}9 \\
20\end{array}$ & $---\overline{2}^{--}$ & I & ---- \\
\hline & 2 & & $\mathrm{~T}$ & & & & & & 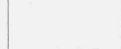 \\
\hline $\begin{array}{l}\text { 4495. } 83 \\
4496.474\end{array}$ & $\begin{array}{r}2 \\
10\end{array}$ & $\ldots$ & I & ---- & $\begin{array}{l}4561.12 \\
4561.57\end{array}$ & $\begin{array}{l}2 \\
2\end{array}$ & $-\ldots--$ & I & $\cdots$ \\
\hline 4497. 52 & 1 & - & I & $\cdots$ & $\begin{array}{l}4001.87 \\
4561.84\end{array}$ & 1 & - n- & I & - \\
\hline 4498. 94 & 8 & $\cdots--$ & I & $-\ldots$ & 4562. 638 & 15 & 1 & $\mathrm{I}$ & 7 \\
\hline 4499. 662 & 50 & 5 & $\mathrm{I}$ & 3 & 4563. 75 & 1 & $30 \mathrm{hl}$ & II & 7 \\
\hline 4501. 49 & 3 & $\ldots$ & I & $\ldots \ldots$ & 4565. 95 & 300 & 50 & I & 1 \\
\hline 4501.78 & $2 \mathrm{~h}$ & $\ldots--$ & I & $\ldots-$ & 4569.910 & 7 & $\ldots-\ldots$ & I & $\ldots$ \\
\hline 4502.01 & 4 & $\ldots \ldots$ & $\mathrm{I}$ & $\ldots$ & 4570.510 & 8 & & $\mathrm{I}$ & $\ldots$ \\
\hline 4503. 095 & 15 & $\ldots \ldots$ & $\mathrm{I}$ & $-\ldots$ & 4570.680 & 10 & $150 \mathrm{hl}$ & II & 4 \\
\hline 4504.01 & 1 & $\ldots-\cdots$ & I & $\ldots$ & 4573.80 & 20 & 100 & II & 4 \\
\hline 4504. 328 & 4 & - & I & - - - & 4575.05 & 1 & 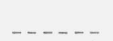 & I & $\ldots \ldots$ \\
\hline 4506. 669 & 15 & 2 & $\mathrm{I}$ & $\ldots$ & 4576. 60 & 1 & $\ldots \ldots$ & I & $\ldots \ldots$ \\
\hline 4508. 874 & 2 & 5 & II & $\ldots \ldots$ & 4577.06 & 1 & $\ldots \ldots$ & I & $\ldots .-$ \\
\hline 4510.52 & 1 & $\ldots \ldots$ & I & $-\ldots-$ & 4577.78 & 2 & $\ldots-\ldots$ & I & $\ldots$. \\
\hline 4510.96 & 2 & $\ldots \ldots$ & I & $\ldots$ & 4578. 18 & 2 & $\ldots \ldots$ & I & $-\ldots$ \\
\hline 4511.970 & 3 & $\ldots$ & $\mathrm{I}$ & $\ldots \ldots$ & 4579.05 & 2 & - & $\mathrm{I}$ & $\ldots \ldots$ \\
\hline 4512.51 & 1 & $\ldots$ & I & $\ldots$ & 4579.38 & 8 & ...... & I & $\ldots$ \\
\hline 4512.60 & 1 & - - . - & I & $\ldots \ldots$ & 4581. 030 & 10 & - - - - - & I & $\ldots \ldots$ \\
\hline 4512.87 & 1 & $\ldots \ldots$ & I & $\ldots-\ldots$ & 4582.08 & 1 & $\ldots \ldots$ & $\mathrm{I}$ & $\ldots \ldots$ \\
\hline 4513.62 & $2 \mathrm{~h}$ & $\ldots \ldots$ & $\mathrm{I}$ & $\ldots$ & 4582.26 & 1 & $\ldots \ldots$ & I & $\ldots$ \\
\hline 4514. 35 & 2 & - & I & & 4583.53 & 1 & & I & - - - - \\
\hline 4514. 603 & 5 & $\ldots-\ldots$ & $\mathrm{I}$ & $\ldots-$ & 4583. 86 & 2 & - n- & I & $-\ldots$ \\
\hline 4517. 068 & 10 & $\ldots$ & I & $\ldots$ & 4585.00 & 1 & - . & I & $\ldots$ \\
\hline 4517. 32 & 3 & $-\cdots--$ & I & $\ldots \ldots$ & 4585. 10 & 2 & $--\ldots-\ldots$ & $\mathrm{I}$ & $\ldots$ \\
\hline 4518. 305 & 50 & 5 & I & 3 & 4586. 240 & 20 & 70 & II & 6 \\
\hline 4518.98 & 3 & $60 \mathrm{~h}$ & II & $6 ?$ & 4588. 22 & 5 & $\ldots \ldots$ & I & $\ldots--$ \\
\hline 4519. 69 & 2 & $\ldots$ & I & $\ldots \ldots$ & 4588. 81 & 1 & $\ldots \ldots$ & I & $\ldots$ \\
\hline 4520.587 & 30 & 4 & I & 7 & 4589. 13 & 3 & - & I & $\ldots$ \\
\hline 4521. 477 & 4 & $-\cdots-$ & I & $\ldots-$ & 4590.537 & 40 & 4 & I & $\ldots-\ldots$ \\
\hline 4522. 182 & 8 & 1 & $\mathrm{I}$ & $\ldots$. & 4592.70 & 1 & $\ldots \ldots$ & I & $\ldots \ldots$ \\
\hline 4523.32 & 4 & $\ldots$ & I & $\ldots \ldots$ & 4597.945 & 60 & 6 & $\mathrm{I}$ & 7 \\
\hline 4523.575 & 4 & & $\mathrm{I}$ & $\ldots$ & 4598. 801 & 300 & 30 & $\mathrm{I}$ & 2 \\
\hline 4524. 728 & 10 & 200 & II & 6 & 4598. 92 & 200 & 20 & $\mathrm{I}$ & \\
\hline 4525. 92 & 15 & 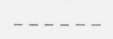 & I & $\ldots \ldots$ & 4599.44 & 15 & 150 & II & 6 \\
\hline 4527.31 & 1 & $\ldots \ldots$ & I & $\ldots$. & 4602.61 & 2 & $40 \mathrm{hl}$ & II & 4 \\
\hline 4527.67 & 1 & $\ldots$ & I & $\ldots \ldots$ & 4602. 75 & 30 & 4 & I & $\ldots$ \\
\hline 4527.95 & 2 & $\ldots \ldots$ & $\mathrm{I}$ & $\ldots$ & 4603.524 & 15 & $-\ldots-\ldots$ & $\mathrm{I}$ & $\ldots-\ldots$ \\
\hline 4530. 133 & 12 & $\ldots \ldots$ & I & $-\ldots$ & 4604.97 & $3 \mathrm{hl}$ & $\ldots \ldots$ & $\mathrm{I}$ & $\ldots \ldots$ \\
\hline 4531. 14 & 1 & $\ldots---$ & I & $\ldots \ldots$ & 4605. 309 & 4 & $---\ldots$ & $\mathrm{I}$ & $-\cdots-\cdots$ \\
\hline 4531.87 & $1 d$ & - . . - & I & $-\ldots$ & 4605. 782 & 20 & 80 & II & 5 \\
\hline 4532. 74 & 2 & & I & & 4606. 07 & $4 \mathrm{hl}$ & ---- & $\mathrm{I}$ & ---- \\
\hline 4533. 165 & 30 & 100 & II & 5 & 4606. 75 & 6 & $-\cdots-\bar{T}$ & $\mathrm{I}$ & 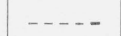 \\
\hline 4534. 77 & 1 & -10 & I & $\cdots-\infty$ & 4606. 91 & 5 & $20 \mathrm{Hl}$ & II & $\ldots$. \\
\hline 4535. 37 & 10 & 100 & II & 5 & 4607. 48 & $5 \mathrm{~h}$ & $---1 \overline{1}$ & I & $-\cdots$ \\
\hline 4537.548 & 10 & ----- & 1 & $-\cdots$ & 4608.093 & 100 & 10 & 1 & 1 \\
\hline 4539. 76 & 3 & 7 & II & & 4610. 288 & 2 & $--\cdots$ & $\mathrm{I}$ & $\ldots-$ \\
\hline 4540.934 & 200 & 20 & I & 7 & 4611.63 & $1 d$ & $\ldots \ldots$ & I & $\ldots \ldots$ \\
\hline 4541. 29 & 10 & 60 & II & 6 & 4612. 29 & 9 & $\ldots \ldots$ & I & $\ldots$ \\
\hline 4541. 71 & 40 & 4 & I & $\ldots-$ & 4613. 222 & 3 & $\ldots-\ldots$ & $\mathrm{I}$ & $\ldots$ \\
\hline 4543.01 & 20 & 3 & I & $\ldots$. & 4613.744 & 15 & 300 & II & 6 \\
\hline 4544. 02 & 100 & 10 & I & 7 & 4614. 192 & 40 & 4 & I & \\
\hline 4544. 775 & 1 & ----- & I & $\ldots$ & 4614.60 & 10 & 1 & $\mathrm{I}$ & $\ldots \ldots$ \\
\hline 4546. 90 & 10 & 1 & I & $\ldots-$ & 4617. 78 & 2 & - - - - & $\vec{I}$ & $\ldots \ldots$ \\
\hline 4546.98 & 15 & 2 & I & 7 & 4619.52 & 20 & 2 & I & 7 \\
\hline 4547.87 & 30 & 2 & $\mathrm{I}$ & 3 & 4620.862 & 300 & 30 & $\mathrm{I}$ & 2 \\
\hline
\end{tabular}


TABLE 1. Emission spectra of hafnium-Continued

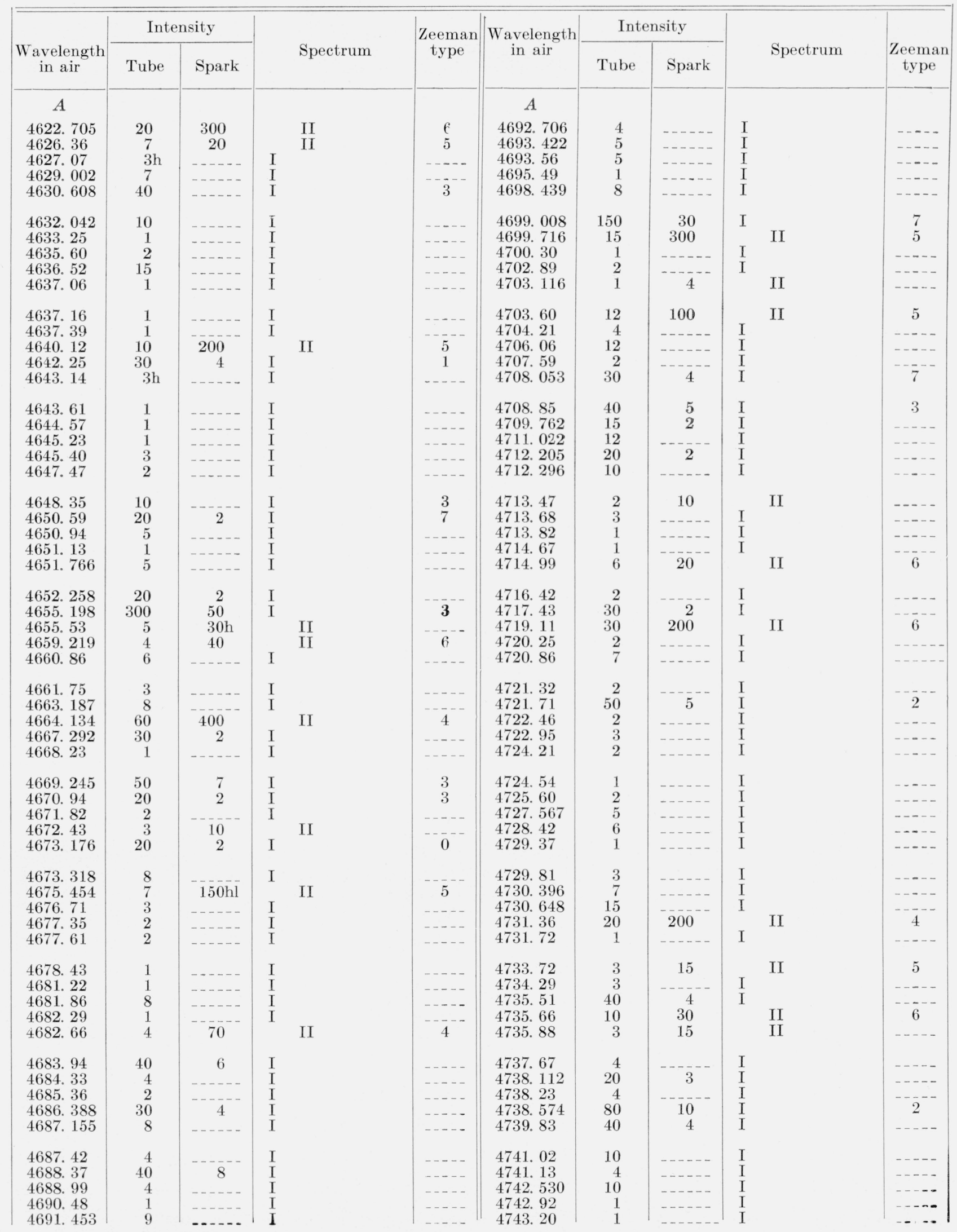


TABLE 1. Emission spectra of hafnium-Continued

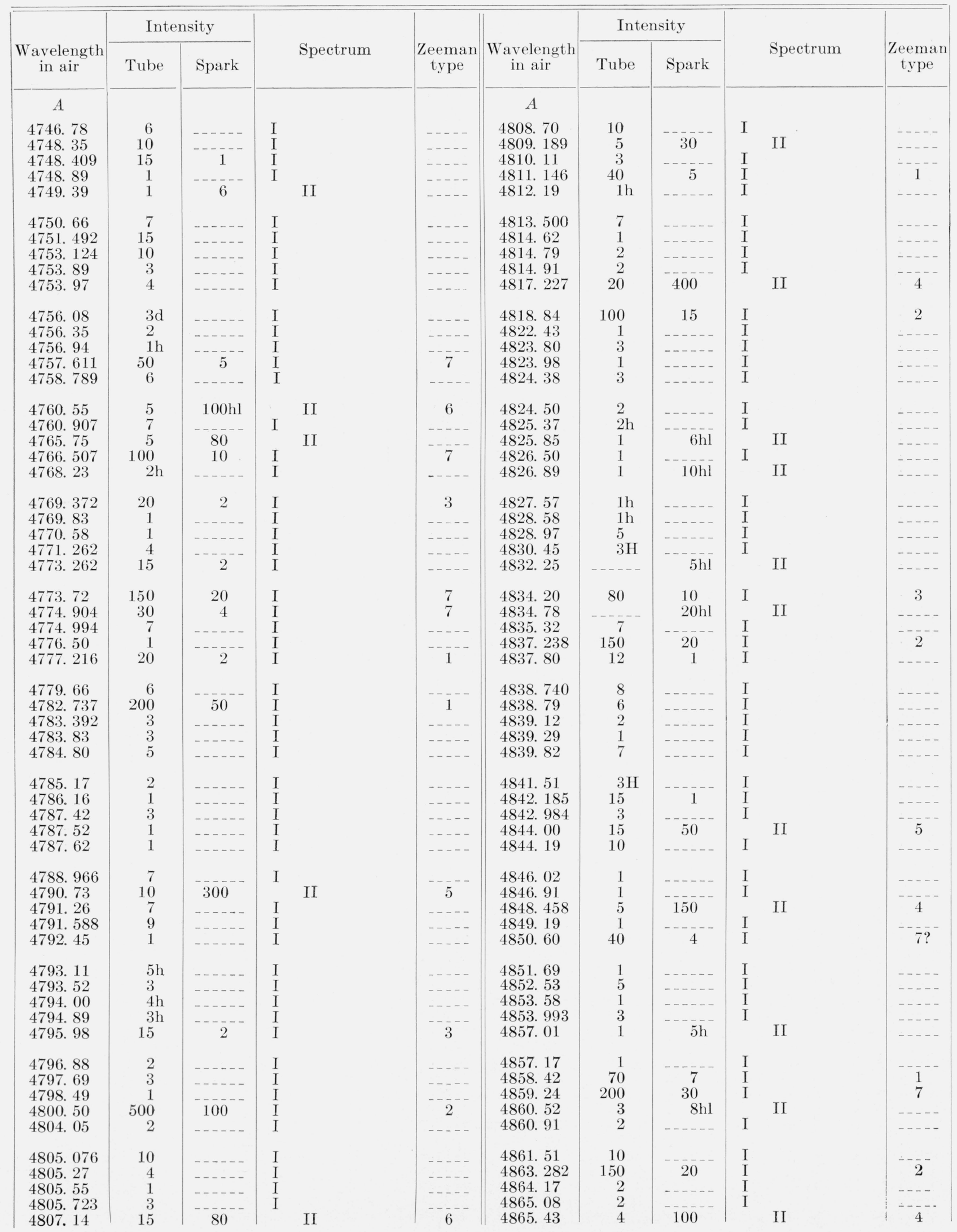


TABLE 1. Emission spectra of hafnium - Continued

\begin{tabular}{|c|c|c|c|c|c|c|c|c|c|c|}
\hline \multirow[b]{2}{*}{$\begin{array}{l}\text { Wavelength } \\
\text { in air }\end{array}$} & \multicolumn{2}{|c|}{ Intensity } & \multirow[b]{2}{*}{ Spectrum } & \multirow[b]{2}{*}{$\begin{array}{c}\text { Zeeman } \\
\text { type }\end{array}$} & \multirow[b]{2}{*}{$\begin{array}{c}\text { Wavelength } \\
\text { in air }\end{array}$} & \multicolumn{2}{|c|}{ Intensity } & \multirow{2}{*}{\multicolumn{2}{|c|}{ Spectrum }} & \multirow[b]{2}{*}{$\begin{array}{c}\text { Zeeman } \\
\text { type }\end{array}$} \\
\hline & Tube & Spark & & & & Tube & Spark & & & \\
\hline$A$ & & & & & $A$ & & & & & \\
\hline 4865.546 & 2 & $\ldots \ldots$ & I & $\ldots$ & 4925. 214 & 15 & 1 & I & & $\ldots \ldots$ \\
\hline 4865.82 & 1 & $\ldots$ & I & $-\cdots$ & 4926. 189 & 30 & 2 & I & & $\ldots-$ \\
\hline 4866.33 & 3 & - . . . - & I & $\ldots$ & 4926. 594 & 10 & 2 & I & & $\ldots$ \\
\hline 4868. 84 & 1 & ------ & $\mathrm{I}$ & & 4926. 983 & 8 & 30 & & II & 4 \\
\hline 4872. 949 & 50 & 5 & I & 1 & 4927. 15 & 2 & $\ldots-\ldots$ & I & & $\ldots-$ \\
\hline 4875. 63 & 1 & $\ldots$ & I & & 4927. 60 & $2 \mathrm{~h}$ & $\ldots$ & I & & \\
\hline 4875. 76 & 1 & - & I & - n & 4928. 194 & 6 & $-\ldots-1$ & $\mathrm{I}$ & & $\ldots$ \\
\hline 4875. 92 & 2 & $-\ldots-$ & I & 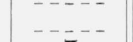 & 4929.55 & 1 & - n- & I & & $-\ldots$ \\
\hline 4877. 587 & 150 & 20 & $\mathrm{I}$ & 7 & 4932. 260 & 7 & - n- & $\mathrm{I}$ & & 2 \\
\hline 4878. 166 & 20 & 4 & I & 7 & 4934. 458 & 30 & 150 & & II & 4 \\
\hline 4881. 27 & 1 & _. - - - & I & $\ldots \ldots$ & 4934. 595 & 6 & - - - - - & I & & $\ldots \ldots$ \\
\hline 4881. 74 & 3 & $\ldots \ldots$ & $\mathrm{I}$ & $\ldots$ & 4936. 35 & 3 & _. & I & & $-\ldots$ \\
\hline 4882. 48 & 2 & $\ldots \ldots$ & $\mathrm{I}$ & 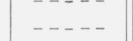 & 4937. 810 & 5 & $\cdots \ldots$ & I & & $-\ldots$ \\
\hline 4882.649 & 2 & $8 \mathrm{hl}$ & II & $-\cdots$ & 4938. 717 & 3 & 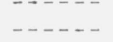 & I & & $-\cdots$ \\
\hline 4883. 21 & 1 & $15 \mathrm{hl}$ & II & --- & 4942. 74 & 1 & $\ldots \ldots$ & $\mathrm{I}$ & & $\ldots$ \\
\hline 4883. 66 & 2 & $\ldots \ldots$ & I & $\ldots$ & 4943. 42 & 15 & 2 & I & & 7 \\
\hline 4884. 258 & 10 & $\ldots$ & $\mathrm{I}$ & $\ldots$ & 4944.82 & 10 & 1 & $\mathrm{I}$ & & \\
\hline 4884.65 & 10 & & I & $-\ldots$ & 4945. 370 & 7 & 40 & & II & 5 \\
\hline 4885. 69 & 2 & $40 \mathrm{hl}$ & II & 4 & 4945. 84 & 3 & & I & & \\
\hline 4886.47 & 4 & $\ldots \ldots$ & I & $\ldots \ldots$ & 4947. 336 & 15 & 2 & I & & 3 \\
\hline 4886. 84 & 1 & - . . - & I & & 4948. 951 & 100 & 15 & I & & 3 \\
\hline 4887. 73 & 1 & $\ldots$ & $\mathrm{I}$ & - n & 4950.02 & $3 \mathrm{~h}$ & $\ldots \ldots$ & I & & $\ldots$ \\
\hline 4888. 05 & 3 & - & I & $-\ldots$ & 4950.36 & 1 & $-\ldots$ & I & & 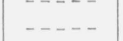 \\
\hline 4889. 609 & 7 & 1 & I & $\ldots$ & 4950.69 & 2 & - & I & & $\ldots$ \\
\hline 4889.932 & 20 & 2 & I & 2 & 4951.095 & 4 & $\ldots$ & I & & $\ldots$. \\
\hline 4890. 754 & 6 & $\ldots \ldots$ & I & -.... & 4955. 65 & 1 & - . . . - & I & & $\ldots \ldots$ \\
\hline 4892. 993 & 5 & $\ldots \ldots$ & I & $\ldots \ldots$ & 4955. 94 & 1 & $\ldots \ldots$ & I & & $\ldots \ldots$ \\
\hline 4893. 62 & 2 & $\ldots$ & $\mathrm{I}$ & $-\ldots$ & 4956. 813 & 5 & - - & I & & $\ldots$ \\
\hline 4894. 82 & 1 & $-\ldots$ & I & $-\ldots$ & 4958. 24 & 2 & $-\ldots--$ & I & & $-\ldots$ \\
\hline 4895. 086 & 5 & -.... & $\bar{I}$ & $\ldots$ & 4960.074 & 5 & -...- & I & & $\ldots$ \\
\hline 4895. 301 & 6 & - - - - & I & & 4960. 32 & 1 & & I & & $-\ldots$ \\
\hline 4895. 48 & 1 & $\ldots \ldots$ & I & $-\ldots$ & 4960.53 & & $4 \mathrm{~h}$ & & II & $\ldots$ \\
\hline 4896.34 & 40 & 4 & $\vec{I}$ & 3 & 4962.381 & 50 & 6 & I & & 1 \\
\hline 4897. 26 & 2 & ----- & I & $\ldots$ & 4964. 740 & 4 & $\ldots-\ldots$ & I & & $\ldots$ \\
\hline 4898. 38 & 1 & $\ldots . .$. & I & $\ldots$ & 4965.28 & 2 & $\ldots$ & I & & $\ldots$ \\
\hline 4898. 73 & 3 & & I & & 4965. 83 & 2 & - & I & & . _ \\
\hline 4900. 179 & 8 & 1 & I & $\ldots$ & 4966.03 & 1 & 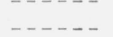 & $\mathrm{I}$ & & $-\ldots$ \\
\hline 4901. 356 & 6 & $-\ldots-\ldots$ & $\mathrm{I}$ & $-1-$ & 4967. 616 & 5 & 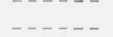 & $\mathrm{I}$ & & $\ldots$ \\
\hline 4901. 44 & 4 & 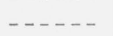 & I & $\ldots \ldots$ & 4975. 26 & 400 & 50 & I & & 7 \\
\hline 4901. 767 & 5 & $\ldots-\ldots$ & I & $\ldots$. & 4978. 724 & 4 & $--\ldots-$ & I & & $\ldots$. \\
\hline 4903. 06 & 20 & 2 & I & 2 & 4981. 73 & 2 & - - & I & & $\ldots \ldots$ \\
\hline 4904. 480 & 7 & $300 \mathrm{~h}$ & II & 4 & 4982. 72 & $2 \mathrm{~h}$ &.---- & $\mathrm{I}$ & & $\ldots$ \\
\hline 4906. 17 & 8 & 1 & I & & 4983. 07 & $1 \mathrm{~h}$ &.--- & I & & $\ldots .$. \\
\hline 4906. 400 & 6 & 2 & $\mathrm{I}$ & 7 & 4983. 70 & $3 \mathrm{~h}$ & . & $\mathrm{I}$ & & $-\ldots$ \\
\hline 4907. 079 & 6 & $\ldots \ldots$ & $\mathrm{I}$ & $\ldots$. & 4984. 766 & 3 & 15 & & II & 6 \\
\hline 4907. 244 & 6 & & I & & 4985. 17 & 2 & - . - . & I & & $\ldots \ldots$ \\
\hline 4907. 33 & 3 & $15 \mathrm{~h}$ & II & 6 & 4985. 33 & 3 & $\ldots \ldots$ & I & & $\ldots . .$. \\
\hline 4907. 56 & 3 & - - - - - & I & $\ldots \ldots$ & 4986. 37 & 1 & $\ldots \ldots$ & I & & $\ldots \ldots$ \\
\hline 4908. 30 & 1 & $\ldots$ & $\mathrm{I}$ & 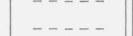 & 4986.55 & 1 & - & $\mathrm{I}$ & & $\ldots$ \\
\hline 4910.10 & 30 & 2 & $\mathrm{I}$ & 2 & 4987. 28 & 1 & $\ldots$ & $\mathrm{I}$ & & $\ldots$ \\
\hline 4911. 40 & 1 & - & $\mathrm{I}$ & & 4988. 712 & 2 & & I & & - \\
\hline 4912.11 & 5 & $\ldots \ldots$ & I & $\ldots$ & 4988. 88 & 1 & $\ldots \ldots$ & I & & $\ldots$ \\
\hline 4914. 888 & 7 & - & I & $\ldots$ & 4991. 06 & 1 & $\ldots$ & $\mathrm{I}$ & & $\ldots$ \\
\hline 4915. 32 & 40 & 6 & I & 3 & 4994. 795 & $4 \mathrm{~h}$ & $\ldots \ldots$ & $\mathrm{I}$ & & $\ldots$ \\
\hline 4916. 98 & 8 & $\ldots-\ldots$ & I & $\ldots$ & 4995. 831 & 2 & $\ldots \ldots$ & I & & $\ldots$ \\
\hline 4918. 094 & 4 & - . & I & - - - & 4998. 03 & $4 \mathrm{hs}$ & $\ldots \ldots$ & I & & $\ldots$ \\
\hline 4920. 16 & 2 & $-\ldots$ & I & $\ldots$ & 4998. 76 & 1 & $\ldots \ldots$ & I & & $\ldots \ldots$ \\
\hline 4920.95 & 10 & $40 \mathrm{Hl}$ & II & 7 & 4999. 21 & 7 & $\ldots-$ & I & & \\
\hline 4923. 92 & 2 & - & I & $\ldots$ & 4999. 702 & 30 & 100 & & II & 5 \\
\hline 4924.54 & 1 & $\ldots \ldots$ & I & $\ldots$. & 5000.54 & 20 & 2 & I & & 3 \\
\hline
\end{tabular}


TABLE 1. Emission spectra of hafnium-Continued

\begin{tabular}{|c|c|c|c|c|c|c|c|c|c|}
\hline \multirow[b]{2}{*}{$\begin{array}{l}\text { Wavelength } \\
\text { in air }\end{array}$} & \multicolumn{2}{|c|}{ Intensity } & \multirow[b]{2}{*}{ Spectrum } & \multirow[b]{2}{*}{$\begin{array}{c}\text { Zeeman } \\
\text { type }\end{array}$} & \multirow[b]{2}{*}{$\begin{array}{c}\text { Wavelength } \\
\text { in air }\end{array}$} & \multicolumn{2}{|c|}{ Intensity } & \multirow[b]{2}{*}{ Spectrum } & \multirow[b]{2}{*}{$\begin{array}{c}\text { Zeeman } \\
\text { type }\end{array}$} \\
\hline & Tube & Spark & & & & Tube & Spark & & \\
\hline$A$ & & & & & $A$ & & & & \\
\hline 5000.81 & 1 & $\ldots \ldots$ & I & $\ldots \ldots$ & 5082. 21 & 3 & $\ldots \ldots$ & I & $\ldots \ldots$ \\
\hline 5001.96 & 3 & $\ldots \ldots$ & I & $\ldots$ & 5082. 770 & 2 & $\ldots \ldots$ & I & $\ldots-\ldots$ \\
\hline 5002.06 & $2 \mathrm{~h}$ & $\ldots \ldots$ & I & $\ldots \ldots$ & 5084. 74 & 1 & $\ldots \ldots$ & I & - . . . \\
\hline 5004.50 & 1 & $\ldots \ldots$ & I & $\ldots$ & 5085.07 & 2 & $\ldots \ldots$ & I & $\ldots-\ldots$ \\
\hline 5005.18 & 2 & $\ldots \ldots$ & $\mathrm{I}$ & $\ldots$. & 5086. 482 & 4 & $\ldots \ldots$ & I & $\ldots--$ \\
\hline 5007. 21 & 1 & $\ldots \ldots$ & I & $\ldots \ldots$ & 5086.96 & 10 & 1 & I & $\ldots \ldots$ \\
\hline 5007.94 & 2 & $\ldots \ldots$ & $\mathrm{I}$ & $\ldots$ & 5087.37 & 2 & $\ldots+\ldots$ & I & $-\ldots$ \\
\hline 5008.068 & 3 & $\ldots \ldots$ & $\mathrm{I}$ & $\ldots$ & 5087.46 & 2 & - . . . & $\mathrm{I}$ & $\ldots$ \\
\hline 5011.01 & $1 \mathrm{~h}$ & $\ldots \ldots$ & $\mathrm{I}$ & $\ldots \ldots$ & 5089.50 & 1 & $\ldots \ldots$ & I & $\ldots$ \\
\hline 5012.185 & 10 & 1 & $\mathrm{I}$ & $\ldots$ & 5090.876 & 15 & 2 & $\mathrm{I}$ & 3 \\
\hline 5012.89 & $1 \mathrm{~h}$ & & $\mathrm{I}$ & -.... & 5090. 996 & 6 & - & I & $\ldots$ \\
\hline 5013.82 & 1 & $8 \mathrm{Hl}$ & II & $\ldots$ & 5091. 28 & 3 & $\ldots \ldots$ & $\mathrm{I}$ & $\ldots$ \\
\hline 5014.28 & 1 & $\ldots-\ldots$ & I & $\ldots$ & 5093. 698 & 5 & $\ldots \ldots$ & I & $\ldots$. \\
\hline 5018.20 & 200 & 20 & I & 1 & 5094. 117 & 2 & $\ldots \ldots$ & I & $\ldots$ \\
\hline 5021.13 & 40 & 5 & $\mathrm{I}$ & 2 & 5094. 683 & 5 & $\ldots \ldots$ & $\mathrm{I}$ & $\ldots-\ldots$ \\
\hline 5021. 745 & 40 & 5 & $\mathrm{I}$ & 2 & 5096. 21 & 1 & $\ldots \ldots$ & $\mathrm{I}$ & $\ldots \ldots$ \\
\hline 5023. 09 & 70 & 5 & $\mathrm{I}$ & 2 & 5097.40 & 1 & - . . . . & $\mathrm{I}$ & $\ldots$ \\
\hline 5025.91 & 15 & 1 & I & 3 & 5097.74 & 2 & $\ldots \ldots$ & $\mathrm{I}$ & $\ldots \ldots$ \\
\hline 5029.46 & 3 & $\ldots \ldots$ & I & $\ldots$ & 5098.80 & 2 & $\ldots \ldots$ & I & $\ldots-$ \\
\hline 5029.71 & 2 & $\ldots \ldots$ & $\bar{I}$ & $\ldots$ & 5100.64 & 60 & 4 & $\mathrm{I}$ & 1 \\
\hline 5929. 99 & $1 \mathrm{~h}$ & $\ldots \ldots$ & $\mathrm{I}$ & $\ldots \ldots$ & 5101. 668 & 40 & 4 & $\mathrm{I}$ & 1 \\
\hline 5033.32 & 1 & $\ldots$ & $\mathrm{I}$ & $-\ldots$ & 5102. 634 & 2 & $\ldots \ldots$ & $\mathrm{I}$ & $\ldots-\ldots$ \\
\hline 5034.14 & 2 & & $\mathrm{I}$ & $\ldots$ & 5105. 64 & 1 & _. . . . & $\mathrm{I}$ & $\ldots$ \\
\hline 5034. 33 & 4 & 30 & II & 5 & 5107. 319 & 4 & $\ldots \ldots$ & $\mathrm{I}$ & $\ldots$ \\
\hline 5034.92 & 30 & 3 & I & 7 & 5108.55 & 1 & - . - - & I & 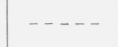 \\
\hline 5035.50 & 1 & $\ldots \ldots$ & I & - . & 5109. 04 & $1 \mathrm{~h}$ & & I & \\
\hline 5035. 899 & 6 & $\ldots \ldots$ & $\mathrm{I}$ & $\ldots$ & 5110.56 & 2 & $50 \mathrm{hl}$ & II & 4 \\
\hline 5037.74 & 9 & $\ldots \ldots$ & I & 7 & 5111.148 & 20 & 2 & I & \\
\hline 5038.08 & 3 & $\ldots \ldots$ & I & $\ldots \ldots$ & 5112.130 & 100 & 10 & $\mathrm{I}$ & 1 \\
\hline 5039.15 & 3 & $\ldots \ldots$ & $\mathrm{I}$ & $\ldots$ & 5117. 09 & 10 & $\ldots \ldots$ & I & ---- \\
\hline 5040.202 & 15 & & I & & 5122. 727 & 4 & & I & $\ldots \ldots$ \\
\hline 5040.815 & 50 & 400 & II & 4 & 5126.81 & 3 & & $\mathrm{I}$ & $\ldots .$. \\
\hline 5042.30 & 1 & $\ldots \ldots$ & $\mathrm{I}$ & $\ldots \ldots$ & 5127.05 & . & $5 \mathrm{~h}$ & II & $\ldots \ldots$ \\
\hline 5043.102 & 2 & $-\ldots-\ldots$ & I & $\ldots \ldots$ & 5128. 116 & 8 & & 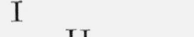 & $\ldots-\ldots$ \\
\hline 5047.440 & 300 & 30 & I & 2 & 5128.504 & 20 & 100 & II & 4 \\
\hline 5049.915 & 1 & $20 \mathrm{~h}$ & II & 4 & 5128.96 & & $6 \mathrm{hl}$ & II & $\ldots \ldots$ \\
\hline 5051. 319 & 50 & 3 & I & 3 & 5130.28 & 1 & $\ldots \ldots$ & I & $\ldots \ldots$ \\
\hline 5053.68 & 2 & $\ldots \ldots$ & $\mathrm{I}$ & $\ldots$ & 5131.38 & 1 & $\ldots \ldots$ & I & $\ldots$ \\
\hline 5055. 93 & 2 & $\ldots \ldots$ & $\mathrm{I}$ & $\ldots$ & 5133.10 & 15 & - - - - & I & $\ldots-\ldots$ \\
\hline 5056.89 & 10 & $\ldots \ldots$ & $\mathrm{I}$ & $\ldots$ & 5133. 66 & 1 & $\ldots . .$. & I & $\ldots$ \\
\hline 5057.037 & 5 & $80 \mathrm{~h}$ & II & 6 & 5135.81 & 5 & & $\mathrm{I}$ & \\
\hline 5058. 166 & 7 & 40 & II & 6 & 5136. 20 & 30 & 2 & I & 1 \\
\hline 5058. 778 & 4 & $\ldots \ldots$ & I & .... & 5136.52 & 2 & 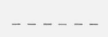 & I & $\ldots$ \\
\hline 5060.584 & 5 & $\ldots \ldots$ & I & $\ldots \ldots$ & 5137.86 & 1 & $\ldots \ldots$ & I & $\ldots$ \\
\hline 5061.80 & $3 \mathrm{~h}$ & $\ldots$ & I & $\ldots$ & 5139.51 & $5 \mathrm{~h}$ & $\ldots . .$. & I & $\ldots$ \\
\hline 5064.15 & $3 \mathrm{~h}$ & $\ldots \ldots$ & I & & 5140. 202 & 4 & $\ldots \ldots$ & I & $\ldots-\ldots$ \\
\hline 5064.67 & 2 & $\ldots \ldots$ & $\mathrm{I}$ & $\ldots$ & 5141.044 & 5 & - - - - & I & $\ldots$ \\
\hline 5067.65 & $2 \mathrm{~h}$ & ----- & $\mathrm{I}$ & $\ldots$ & 5141. 19 & 2 & $\ldots \ldots$ & I & $\ldots \ldots$ \\
\hline 5068.62 & & $7 \mathrm{Hl}$ & II & $\ldots$ & 5141.81 & $2 \mathrm{~h}$ & $\ldots \ldots$ & I & $\ldots$. \\
\hline 5069. 810 & 20 & 1 & I & 1 & 5142. 997 & 9 & $\ldots \ldots$ & I & $-\cdots$ \\
\hline 5071. 21 & 5 & 40 & II & 4 & 5144.30 & 2 & - . - . & I & $\ldots$ \\
\hline 5072.295 & 8 & 1 & I & $\ldots \ldots$ & 5145. 22 & $2 \mathrm{~h}$ & $\ldots \ldots$ & I & $\ldots$ \\
\hline 5073.131 & 4 & $\cdots=-\bar{u}$ & I & $\ldots \ldots$ & 5145.72 & 2 & $-\cdots=-\overline{1}$ & I & $\cdots$ \\
\hline 5074. 72 & 1 & ${ }_{50}^{7 \mathrm{Hl}}$ & II & 6 & 5146. 14 & 1 & $7 \mathrm{hl}$ & II & - - - \\
\hline 5075. 920 & 10 & 50 & II & 6 & 5147.401 & 7 & $\ldots \ldots$ & I & $\cdots$ \\
\hline $5076 \cdot 29$ & 2 & $\ldots \ldots$ & I & $\ldots-$ & 5148. 62 & $1 \mathrm{~h}$ & $\ldots \ldots$ & I & $\cdots$ \\
\hline 5077. 614 & 6 & $\ldots \ldots$ & I & $\ldots$ & 5148. 899 & 8 & $\ldots \ldots$ & I & $\ldots \ldots$ \\
\hline 5079.629 & 20 & 300 & II & 5 & 5153.122 & 40 & 4 & I & 4 \\
\hline 5080.406 & 2 & 40 & II & 4 & 5156.04 & 1 & $50 \mathrm{hl}$ & II & 6 \\
\hline 5081.77 & 10 & 1 & I & $\ldots$ & 5157.21 & 5 & $\ldots \ldots$ & $\mathrm{I}$ & $\ldots \ldots$ \\
\hline
\end{tabular}


TABLe 1. Emission spectra of hafnium-Continued

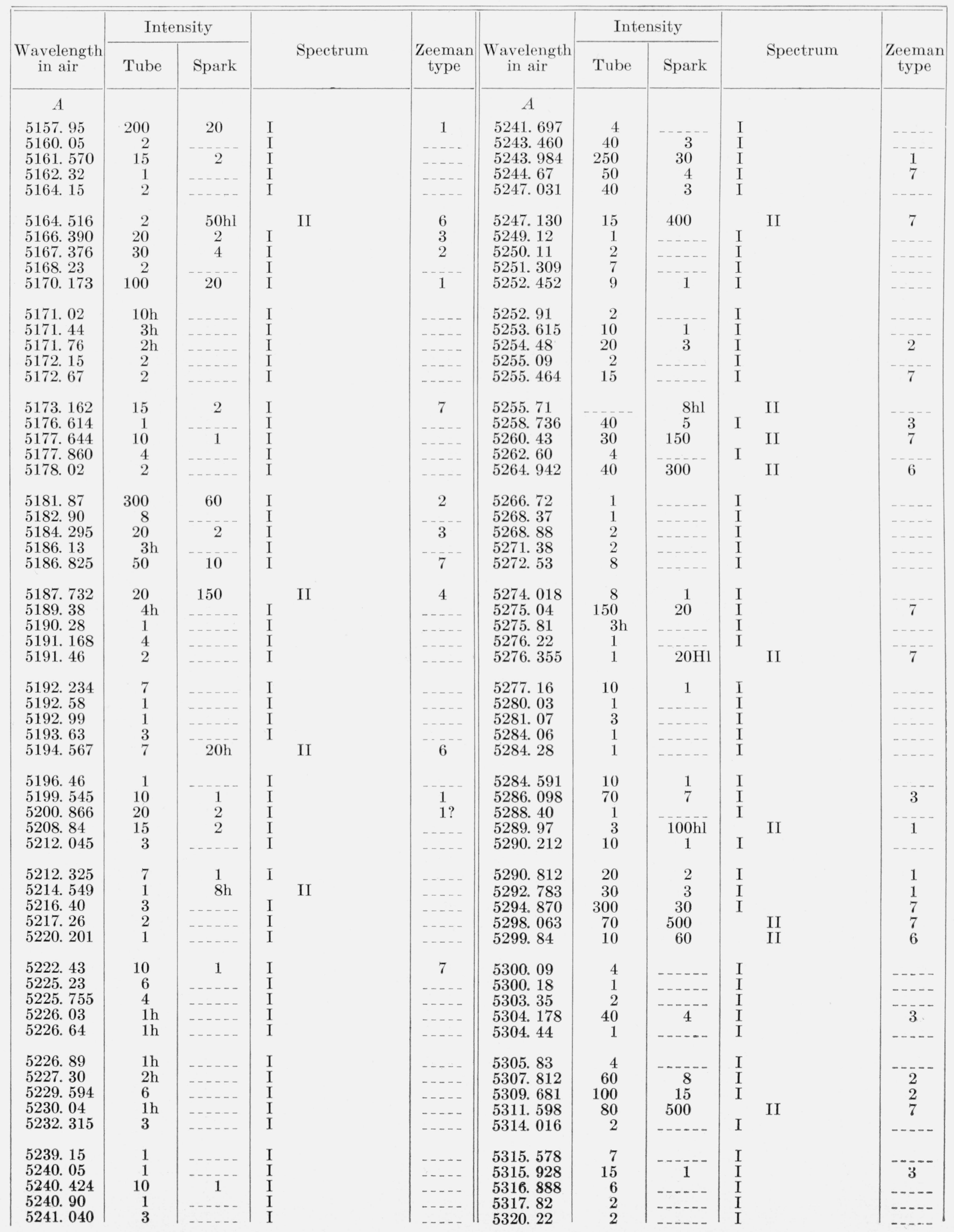


TABLE 1. Emission spectra of hafnium-Continued

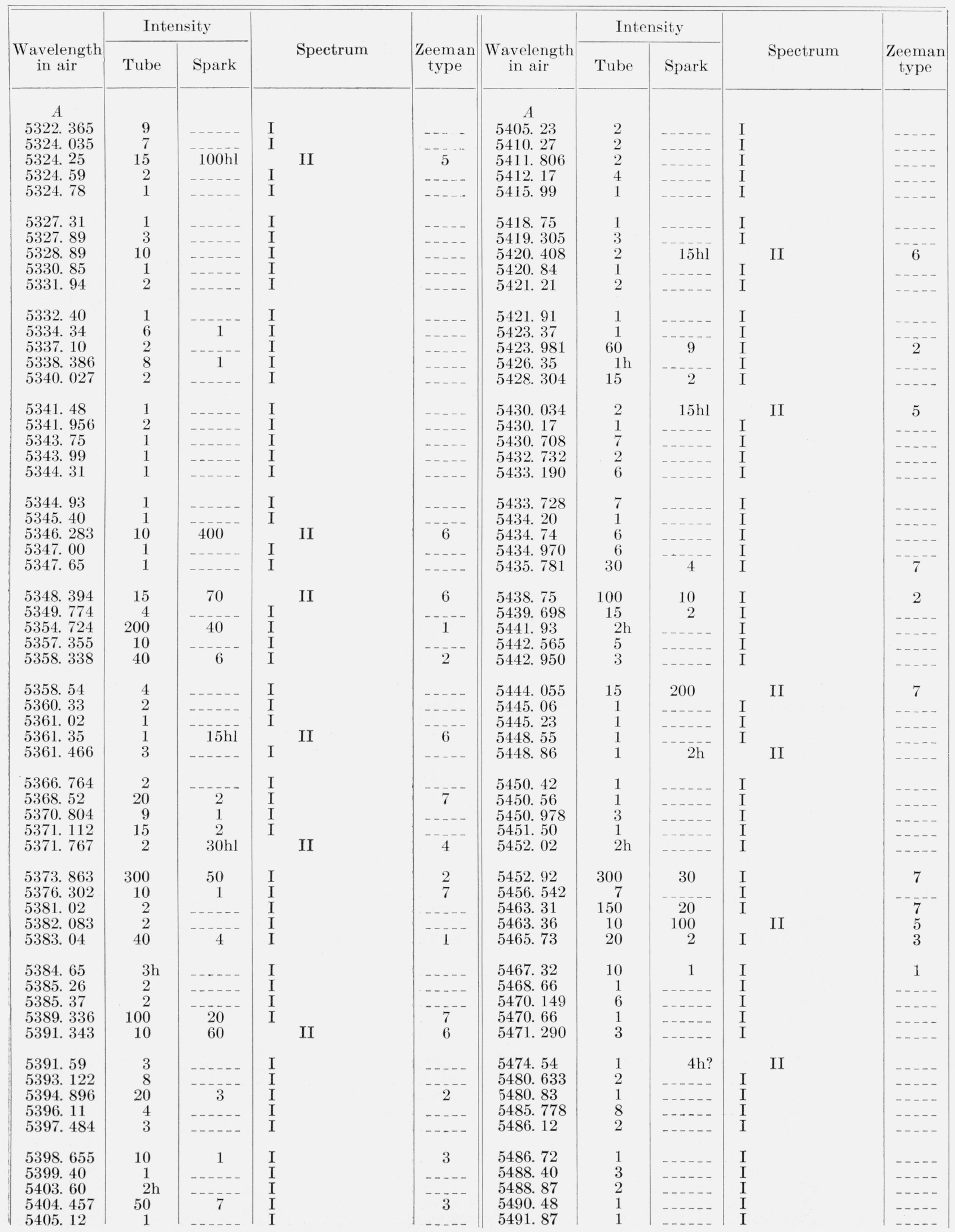


TABLE 1. Emission spectra of hafnium-Continued

\begin{tabular}{|c|c|c|c|c|c|c|c|c|c|}
\hline \multirow[b]{2}{*}{$\begin{array}{l}\text { Wavelength } \\
\text { in air }\end{array}$} & \multicolumn{2}{|c|}{ Intensity } & \multirow[b]{2}{*}{ Spectrum } & \multirow[b]{2}{*}{$\begin{array}{c}\text { Zeeman } \\
\text { type }\end{array}$} & \multirow[b]{2}{*}{$\begin{array}{l}\text { Wavelength } \\
\text { in air }\end{array}$} & \multicolumn{2}{|c|}{ Intensity } & \multirow[b]{2}{*}{ Spectrum } & \multirow[b]{2}{*}{$\begin{array}{c}\text { Zeeman } \\
\text { type }\end{array}$} \\
\hline & Tube & Spark & & & & Tube & Spark & & \\
\hline \begin{tabular}{l}
\multicolumn{1}{c}{$A$} \\
5492.287 \\
5492.56 \\
5492.89 \\
5493.212 \\
5494.54
\end{tabular} & $\begin{array}{l}3 \\
1 \\
1 \\
3 \\
2\end{array}$ & $60 \mathrm{hl}$ & $\begin{array}{ll}\text { I } & \\
\text { I } & \\
\text { I } & \\
& \text { II } \\
\text { I } & \end{array}$ & 4 & \begin{tabular}{l}
\multicolumn{1}{c}{$A$} \\
5568.76 \\
5569.61 \\
5572.30 \\
5573.529 \\
5575.158
\end{tabular} & $\begin{array}{l}1 \\
3 \\
4 \\
6 \\
8\end{array}$ & 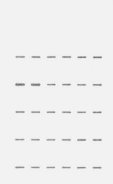 & $\begin{array}{l}\mathrm{I} \\
\mathrm{I} \\
\mathrm{I} \\
\mathrm{I} \\
\mathrm{I}\end{array}$ & $\begin{array}{l}-\cdots- \\
\cdots-1 \\
-\cdots \\
-\cdots- \\
-\cdots-\end{array}$ \\
\hline $\begin{array}{l}5497.301 \\
5498.70 \\
5501.01 \\
5501.53 \\
5502.63\end{array}$ & $\begin{array}{r}50 \\
1 \\
1 \\
4 \\
10\end{array}$ & $\begin{array}{c}5 \\
----- \\
------ \\
----\end{array}$ & $\begin{array}{l}\text { I } \\
\text { I } \\
\text { I } \\
\text { I } \\
\text { I }\end{array}$ & $\begin{array}{c}2 \\
\cdots- \\
\cdots \\
\cdots\end{array}$ & $\begin{array}{l}5575.857 \\
5578.008 \\
5578.35 \\
5579.90 \\
5580.148\end{array}$ & $\begin{array}{r}200 \\
15 \\
1 \\
1 \\
8\end{array}$ & $\begin{array}{c}30 \\
2 \\
-\ldots-- \\
-\ldots--- \\
-\ldots\end{array}$ & $\begin{array}{l}\text { I } \\
\text { I } \\
\text { I } \\
\text { I } \\
\text { I }\end{array}$ & $\begin{array}{c}3 \\
3 \\
--- \\
-\cdots- \\
-\cdots-\end{array}$ \\
\hline $\begin{array}{l}5503.294 \\
5506.20 \\
5510.116 \\
5510.436 \\
5512.302\end{array}$ & $\begin{array}{r}20 \\
1 \\
60 \\
40 \\
10\end{array}$ & $\begin{array}{c}2 \\
-6 \\
7 \\
1\end{array}$ & $\begin{array}{l}\text { I } \\
\text { I } \\
\text { I } \\
\text { I } \\
\text { I }\end{array}$ & 1 & $\begin{array}{l}5580.69 \\
5581.45 \\
5583.934 \\
5585.013 \\
5589.54\end{array}$ & $\begin{array}{r}1 \\
9 \\
10 \\
2 \\
1\end{array}$ & $\begin{array}{c}1 \\
1 \\
-\cdots---\end{array}$ & $\begin{array}{l}\text { I } \\
\text { I } \\
\text { I } \\
\text { I } \\
\text { I }\end{array}$ & $\begin{array}{l}---- \\
---- \\
---1 \\
--- \\
----\end{array}$ \\
\hline $\begin{array}{l}5513.28 \\
5513.97 \\
5514.998 \\
5516.75 \\
5517.09\end{array}$ & $\begin{array}{l}1 \\
1 \\
3 \\
1 \\
2\end{array}$ & 10 & $\begin{array}{ll}\mathrm{I} & \\
\mathrm{I} & \mathrm{II} \\
\mathrm{I} & \\
\mathrm{I} & \end{array}$ & 5 & $\begin{array}{l}5590.691 \\
5594.98 \\
5595.60 \\
5595.73 \\
5597.546\end{array}$ & $\begin{array}{l}3 \\
1 \\
5 \\
3 \\
8\end{array}$ & $\begin{array}{r}9 \mathrm{hl} \\
-\ldots--- \\
-\ldots--- \\
-\ldots-\ldots\end{array}$ & $\begin{array}{ll} & \text { II } \\
\text { I } \\
\text { I } \\
\text { I } \\
\text { I }\end{array}$ & $\begin{array}{c}6 \\
--- \\
\cdots-- \\
\cdots \cdots\end{array}$ \\
\hline $\begin{array}{l}5517.73 \\
5518.443 \\
5520.01 \\
552175 \\
5522.05\end{array}$ & $\begin{array}{r}1 \\
10 \\
10 \\
5\end{array}$ & $\begin{array}{c}1 \\
1 \\
2 \mathrm{~h}\end{array}$ & $\begin{array}{ll}\text { I } & \\
\text { I } & \\
\text { I } & \\
\text { I } & \\
& \text { II }\end{array}$ & 1 & $\begin{array}{l}5599.08 \\
5599.42 \\
5600.192 \\
5600.762 \\
5601.15\end{array}$ & $\begin{array}{r}5 \\
1 \\
6 \\
60 \\
3\end{array}$ & $\begin{array}{c}-\cdots-- \\
-\cdots \\
-\cdots--\end{array}$ & $\begin{array}{l}\text { I } \\
\text { I } \\
\text { I } \\
\text { I } \\
\text { I }\end{array}$ & ${ }^{2}$ \\
\hline $\begin{array}{l}5522.554 \\
5522.84 \\
5523.55 \\
5524.348 \\
5524.95\end{array}$ & $\begin{array}{r}20 \\
1 \\
3 \\
40 \\
10\end{array}$ & $\begin{array}{c}2 \\
-200 \\
1\end{array}$ & $\begin{array}{ll}\text { I } & \\
\text { I } & \\
\text { I } & \\
& \text { II } \\
\text { I } & \end{array}$ & $\begin{array}{c}7 \\
-5 \\
-\end{array}$ & $\begin{array}{l}5602.03 \\
5606.07 \\
5606.613 \\
5609.05 \\
5609.38\end{array}$ & $\begin{array}{r}1 \\
1 \\
20 \\
2 \\
2\end{array}$ & ${ }^{-1}$ & $\begin{array}{l}\text { I } \\
\text { I } \\
\text { I } \\
\text { I } \\
\text { I }\end{array}$ & 3 \\
\hline $\begin{array}{l}5525.01 \\
5525.631 \\
5529.01 \\
5530.268 \\
5531.19\end{array}$ & $\begin{array}{r}10 \\
7 \\
20 \\
30 \\
1\end{array}$ & $\begin{array}{c}1 \\
-\cdots \\
-\cdots--\end{array}$ & $\begin{array}{l}\text { I } \\
\text { I } \\
\text { I } \\
\text { I } \\
\text { I }\end{array}$ & 7 & $\begin{array}{l}5611.61 \\
5613.259 \\
5614.004 \\
5615.63 \\
5615.84\end{array}$ & $\begin{array}{r}6 \\
400 \\
80 \\
2 \\
1\end{array}$ & $\begin{array}{c}70 \\
10 \\
-\cdots---\end{array}$ & $\begin{array}{l}\mathrm{I} \\
\mathrm{I} \\
\mathrm{I} \\
\mathrm{I} \\
\mathrm{I}\end{array}$ & $\begin{array}{c}7 \\
? \\
\cdots \\
--\end{array}$ \\
\hline $\begin{array}{l}\text { 5531. } 334 \\
5532.23 \\
5534.638 \\
5535.31 \\
5535.47\end{array}$ & $\begin{array}{l}5 \\
5 \\
8 \\
1 \\
2\end{array}$ & $\begin{array}{c}-\cdots \\
1 \\
-\ldots-\cdots\end{array}$ & $\begin{array}{l}\text { I } \\
\text { I } \\
\text { I } \\
\text { I } \\
\text { I }\end{array}$ & 3 & $\begin{array}{l}5618.57 \\
5624.45 \\
5625.66 \\
5628.293 \\
5630.10\end{array}$ & $\begin{array}{r}2 \\
1 \\
1 \\
40 \\
1\end{array}$ & $\begin{array}{c}----- \\
-\cdots \\
-\cdots---\end{array}$ & $\begin{array}{l}\text { I } \\
\text { I } \\
\text { I } \\
\text { I } \\
\text { I }\end{array}$ & $\frac{\cdots}{\cdots}$ \\
\hline $\begin{array}{l}5538.023 \\
5538.275 \\
5538.559 \\
5539.51 \\
5541.03\end{array}$ & $\begin{array}{r}200 \\
150 \\
20 \\
1 \\
1\end{array}$ & $\begin{array}{r}20 \\
15 \\
2 \\
- \\
\end{array}$ & $\begin{array}{l}\text { I } \\
\text { I } \\
\text { I } \\
\text { I } \\
\text { I }\end{array}$ & $\begin{array}{l}-\ldots- \\
-\cdots \\
-\cdots- \\
-\cdots- \\
-\cdots-\end{array}$ & $\begin{array}{l}5631.13 \\
5631.319 \\
5631.55 \\
5631.93 \\
5632.84\end{array}$ & $\begin{array}{l}1 \\
2 \\
3 \\
1 \\
3\end{array}$ & $\begin{array}{c}20 \mathrm{hl} \\
4\end{array}$ & $\begin{array}{ll}\text { I } & \text { II } \\
\text { I } & \text { II } \\
\text { I } & \end{array}$ & $\bar{y}^{--}$ \\
\hline $\begin{array}{l}5541.920 \\
5545.26 \\
5545.609 \\
5547.83 \\
5548.127\end{array}$ & $\begin{array}{r}15 \\
1 \\
4 \\
-9\end{array}$ & $\begin{array}{c}2 \\
-8 \text { hl } \\
1\end{array}$ & $\begin{array}{ll}\text { I } & \\
\text { I } & \\
\text { I } & \\
& \text { II }\end{array}$ & \begin{tabular}{c}
3 \\
$-\cdots$ \\
\hdashline$-\ldots$ \\
$-\ldots-$
\end{tabular} & $\begin{array}{l}5633.02 \\
5633.450 \\
5633.95 \\
5634.45 \\
5635.81\end{array}$ & $\begin{array}{c}2 \\
20 \\
10 \\
3 \mathrm{~h} \\
2 \mathrm{~h}\end{array}$ & $\begin{array}{c}-{ }^{-} \\
- \\
-\ldots\end{array}$ & $\begin{array}{l}\text { I } \\
\text { I } \\
\text { I } \\
\text { I } \\
\text { I }\end{array}$ & $\begin{array}{c}1 \\
\cdots \\
\cdots\end{array}$ \\
\hline $\begin{array}{l}5550.61 \\
5552.128 \\
5561.69 \\
5561.77 \\
5563.19\end{array}$ & $\begin{array}{r}1000 \\
1000 \\
3 \\
2 \\
3\end{array}$ & $\begin{array}{c}100 \\
100 \\
- \\
-\end{array}$ & $\begin{array}{l}\text { I } \\
\text { I } \\
\text { I } \\
\text { I } \\
\text { I }\end{array}$ & $\begin{array}{c}3 \\
1 \\
\cdots \\
\ldots\end{array}$ & $\begin{array}{l}5635.92 \\
5636.570 \\
5637.555 \\
5638.51 \\
5641.39\end{array}$ & $\begin{array}{l}2 \mathrm{~h} \\
2 \\
2 \\
1 \\
1\end{array}$ & $\begin{array}{c}- \\
8 \mathrm{~h} \\
\ldots \ldots \\
\ldots \ldots\end{array}$ & $\begin{array}{ll}\mathrm{I} & \mathrm{II} \\
\mathrm{I} & \\
\mathrm{I} & \end{array}$ & 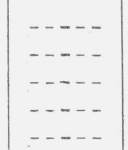 \\
\hline $\begin{array}{l}5563.32 \\
5565.54 \\
5566.87 \\
5567.22 \\
5568.44\end{array}$ & $\begin{array}{l}2 \\
2 \\
2 \\
1 \\
2\end{array}$ & $\begin{array}{r}20 \mathrm{hl} \\
-\end{array}$ & $\begin{array}{ll}\text { I } & \\
& \text { II } \\
\text { I } & \\
\text { I } & \\
\text { I } & \end{array}$ & $\begin{array}{c}-{ }^{--} \\
---\end{array}$ & $\begin{array}{l}5642.65 \\
5644.67 \\
5645.61 \\
5648.563 \\
5649.229\end{array}$ & $\begin{array}{r}1 \\
30 \\
3 \\
5 \\
2\end{array}$ & ${ }^{-1}$ & $\begin{array}{l}\text { I } \\
\text { I } \\
\text { I } \\
\text { I } \\
\text { I }\end{array}$ & ${ }_{1}$ \\
\hline
\end{tabular}


TABLE 1. Emission spectra of hafnium-Continued

\begin{tabular}{|c|c|c|c|c|c|c|c|c|c|}
\hline \multirow[b]{2}{*}{$\begin{array}{l}\text { Wavelength } \\
\text { in air }\end{array}$} & \multicolumn{2}{|c|}{ Intensity } & \multirow[b]{2}{*}{ Spectrum } & \multirow[b]{2}{*}{$\begin{array}{c}\text { Zeeman } \\
\text { type }\end{array}$} & \multirow[b]{2}{*}{$\begin{array}{l}\text { Wavelength } \\
\text { in air }\end{array}$} & \multicolumn{2}{|c|}{ Intensity } & \multirow[b]{2}{*}{ Spectrum } & \multirow[b]{2}{*}{$\begin{array}{c}\text { Zeeman } \\
\text { type }\end{array}$} \\
\hline & Tube & Spark & & & & Tube & Spark & & \\
\hline$A$ & & & & & $A$ & & & & \\
\hline 5650.811 & 50 & 6 & I & 3 & 5745.22 & 2 & & I & \\
\hline 5654.645 & 80 & 9 & $\mathrm{I}$ & 3 & 5748.72 & 80 & 9 & I & 3 \\
\hline 5658.777 & 1 & 10hl & II & $\ldots-\cdots$ & 5751.510 & 3 & $\ldots \ldots$ & I & $\ldots-\ldots$ \\
\hline 5660.38 & 1 & $7 \mathrm{hl}$ & II & $\ldots \ldots$ & 5751. 718 & 4 & $\ldots \ldots$ & I & $\ldots \ldots$ \\
\hline 5660.97 & 1 & $\ldots \ldots$ & I & $\ldots-\cdots$ & 5752.18 & 3 & 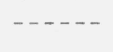 & I & $\ldots--$ \\
\hline 5662. 078 & 40 & 4 & I & 1 & 5752.531 & 2 & $20 \mathrm{hl}$ & II & 6 \\
\hline 5662.982 & 20 & 2 & $\vec{I}$ & 2 & 5756.51 & 3 & $\ldots \ldots$ & I & $\ldots$ \\
\hline 5664. 37 & $\cdots$ & $6 \mathrm{hl}$ & II & $-\cdots-$ & 5756. 83 & 10 & $-\ldots-$ & I & $\ldots-\cdots$ \\
\hline $\begin{array}{l}5664.824 \\
5668.716\end{array}$ & $\begin{array}{r}4 \\
20\end{array}$ & 2 & $\begin{array}{l}\mathrm{I} \\
\mathrm{I}\end{array}$ & $2^{--}$ & $\begin{array}{l}5758.96 \\
5762.41\end{array}$ & $\begin{array}{r}10 \\
2\end{array}$ & $\ldots$ & $\begin{array}{l}\mathrm{I} \\
\mathrm{I}\end{array}$ & -... \\
\hline 5673.565 & 4 & 100hl & II & 7 & 5765.37 & 40 & 4 & I & 2 \\
\hline 5677.210 & 8 & $\ldots \ldots$ & I & $\ldots-$. & 5765.96 & 30 & 4 & I & 7 \\
\hline 5679.553 & 30 & $\ldots \ldots$ & I & 2 & 5766.50 & --- & 2 & II & \\
\hline 5680.444 & 10 & $\ldots \ldots$ & I & $\ldots$ & 5767. 20 & 20 & 60 & II & 6 \\
\hline 5681. 110 & 4 & -.... & I & $-\cdots$ & 5771.397 & 15 & $\ldots \ldots$ & I & $\ldots$ \\
\hline 5682.34 & 2 & - & $\mathrm{I}$ & $\ldots \ldots$ & 5773.33 & 1 & $\ldots$ & I & $\ldots$ \\
\hline 5682.47 & 1 & $\ldots \ldots$ & $\mathrm{I}$ & $\ldots \ldots$ & 5774.016 & 8 & - & I & $\ldots$ \\
\hline 5683.738 & 6 & $\ldots \ldots$ & I & 7 & 5774.89 & 3 & $-\cdots-$ & I & 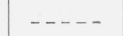 \\
\hline 5684.807 & 15 & 1 & I & $--\cdots$ & 5776.00 & 1 & ---- & I & $\ldots \ldots$ \\
\hline 5686.18 & 1 & $\ldots \ldots$ & I & 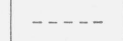 & 5778.92 & 1 & $\ldots \ldots$ & I & $\ldots$ \\
\hline 5688.12 & 15 & & I & $\ldots-$ & 5780.46 & & 7 & II & $\ldots$. \\
\hline 5688.81 & 1 & 2 & II & $\ldots$. & 5784. 75 & $2 \mathrm{~h}$ & $\ldots$ & I & $\ldots$. \\
\hline 5689.77 & 1 & $8 \mathrm{hl}$ & II & $\ldots--$ & 5791. 71 & 10 & $\ldots \ldots$ & I & $\ldots-\ldots$ \\
\hline 5690.41 & 1 & $\ldots \ldots$ & I & $\ldots--$ & 5794. 72 & 10 & ----- & I & ---- \\
\hline 5690.58 & 1 & -...- & I & $\ldots-\cdots$ & 5796. 329 & 30 & 2 & I & $3 ?$ \\
\hline 5691.15 & 1 & $--\ldots-$ & I & $\ldots$ & 5799. 760 & 50 & 5 & I & 3 \\
\hline 5691. 914 & 3 & $\ldots \ldots$ & I & $\ldots-$. & 5800. 567 & 4 & $--=--$ & I & \\
\hline 5692. 72 & 1 & $\ldots \ldots$ & I & $\ldots \ldots$ & 5801. 693 & 5 & 70 & II & 7 \\
\hline $\begin{array}{l}5693.02 \\
5694.34\end{array}$ & $\begin{array}{l}4 \\
5\end{array}$ & $\ldots$ & $\begin{array}{l}\text { I } \\
\text { I }\end{array}$ & $\begin{array}{l}\cdots- \\
\cdots--\end{array}$ & $\begin{array}{l}5801.83 \\
5802.88\end{array}$ & $\begin{array}{r}6 \\
40\end{array}$ & --- & I & 3 \\
\hline 5695. 732 & 8 & 1 & I & & 5803. 64 & 2 & & I & $\ldots-\ldots$ \\
\hline 5697.25 & 15 & 2 & I & 3 & 5804.84 & 1 & $\ldots--$ & I & $\ldots-\ldots$ \\
\hline 5699.46 & 4 & $\ldots \ldots$ & I & $\ldots$ & 5806. 28 & 1 & & I & ...... \\
\hline 5700.82 & 1 & 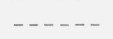 & I & $\ldots-$ & 5808. 428 & 30 & 2 & I & $-\ldots-$ \\
\hline 5701.11 & 4 & $\ldots \ldots$ & I & $\ldots-\cdots$ & 5809.27 & 5 & -- & I & $\ldots-\cdots$ \\
\hline 5702.10 & 20 & 2 & I & 1 & 5809. 499 & 40 & 100 & II & 6 \\
\hline 5703.95 & $2 \mathrm{~h}$ & $\ldots-\ldots$ & $\vec{I}$ & $\ldots \ldots$ & 5810.26 & 2 & $\ldots \ldots$ & I & $\ldots-\ldots$ \\
\hline 5706.64 & 5 & $\ldots \ldots$ & $\vec{I}$ & $\ldots-$. & 5810.58 & 2 & $\ldots \ldots$ & I & $\ldots-$ \\
\hline 5706.85 & 2 & $\ldots \ldots$ & I & $\ldots \ldots$ & 5811. 275 & 15 & 2 & I & $\ldots$. \\
\hline 5708. 38 & 10 & ....- & I & $\cdots-$ & 5813. 14 & 1 & 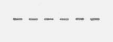 & I & $-\cdots$ \\
\hline 5713. 267 & 100 & 10 & I & 7 & 5816. 499 & 3 & & I & \\
\hline 5719. 175 & 600 & 100 & I & 2 & 5817. 475 & 80 & 6 & I & 3 \\
\hline 5721.68 & 3 & ------ & I & $\ldots-$ & 5817. 920 & 6 & $\ldots \ldots$ & I & $\ldots \ldots$ \\
\hline 5723.880 & 8 & 1 & I & $\ldots$. & 5818. 194 & 4 & $\ldots \ldots$ & I & $\ldots$. \\
\hline 5724.80 & 6 & $\ldots-\ldots$ & I & $-\ldots$ & 5818.58 & 1 & $\ldots \ldots$ & I & $-\cdots$ \\
\hline 5729.683 & 20 & 2 & I & $\ldots \ldots$ & 5837. 05 & 6 & & I & \\
\hline 5734.13 & 10 & 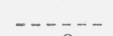 & $\mathrm{I}$ & -..-- & 5838. 896 & 40 & 4 & I & 1 \\
\hline 5734.514 & 50 & 6 & I & $3 ?$ & 5842. 235 & 20 & 300 & II & 4 \\
\hline 5735. 61 & 2 & ---- & I & $\ldots$ & 5844. 879 & 8 & 1 & I & $-\ldots-$ \\
\hline 5735.986 & 9 & 1 & I & $\ldots$ & 5845.866 & 100 & 10 & $\mathrm{I}$ & 2 \\
\hline 5736. 32 & 1 & $\ldots \ldots$ & I & $-\cdots$ & 5847.12 & 3 & & I & \\
\hline 5736.92 & 7 & - - - - & $\mathrm{I}$ & $\ldots-$ & 5847. 768 & 80 & 8 & I & 2 \\
\hline 5737.37 & 1 & 4 & II & $-\cdots$ & 5849.688 & 60 & 8 & I & 1 \\
\hline 5738. 24 & 1 & 2 & II & - . - & 5851.54 & 1 & $\ldots \ldots$ & I & - . - \\
\hline 5739. 915 & 4 & $-\cdots-$ & I & $\ldots-$ & 5853.44 & 1 & -.... & I & $\ldots$ \\
\hline 5740.93 & 1 & $\ldots \ldots$ & I & $-\ldots$ & 5853. 76 & 3 & $\ldots-\ldots$ & I & $\ldots \ldots$ \\
\hline 5743. 49 & 1 & ----- & I & $\ldots-$ & 5856.55 & $1 \mathrm{~h}$ & $-\cdots-$ & I & $-\ldots-$ \\
\hline 5744. 217 & 10 & 1 & I & $-\ldots$ & 5858. 349 & 30 & 3 & I & 2 \\
\hline 5744. 37 & 1 & $-\ldots$ & I & $\ldots--$ & 5859.07 & 4 & $\ldots-\cdots$ & I & $\ldots$ \\
\hline 5745.12 & 1 & - . - . & I & $\ldots$ & 5859. 34 & 3 & $\ldots \ldots$ & I & $\ldots$ \\
\hline
\end{tabular}


TABLE 1. Emission spectra of hafnium-Continued

\begin{tabular}{|c|c|c|c|c|c|c|c|c|c|}
\hline \multirow[b]{2}{*}{$\begin{array}{c}\text { Wavelength } \\
\text { in air }\end{array}$} & \multicolumn{2}{|c|}{ Intensity } & \multirow[b]{2}{*}{ Spectrum } & \multirow[b]{2}{*}{$\begin{array}{c}\text { Zeeman } \\
\text { type }\end{array}$} & \multirow[b]{2}{*}{$\begin{array}{c}\text { Wavelength } \\
\text { in air }\end{array}$} & \multicolumn{2}{|c|}{ Intensity } & \multirow[b]{2}{*}{ Spectrum } & \multirow[b]{2}{*}{$\begin{array}{c}\text { Zeeman } \\
\text { type }\end{array}$} \\
\hline & Tube & Spark & & & & Tube & Spark & & \\
\hline$A$ & & & & & $A$ & & & & \\
\hline 5860.42 & 4 & 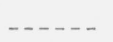 & I & $\ldots-$. & 5971. 91 & 2 & & I & \\
\hline 5862.07 & 2 & $\ldots \ldots$ & $\mathrm{I}$ & $-\ldots$ & 5974. 294 & 200 & 30 & $\mathrm{I}$ & $2^{--}$ \\
\hline 5864.32 & 1 & _..... & I & $\ldots \ldots$ & 5974. 728 & 80 & 8 & $\mathrm{I}$ & 1 \\
\hline 5868.195 & 8 & $\ldots \ldots$ & I & $\ldots-$ & 5978.67 & 150 & 20 & I & 7 \\
\hline 5868. 39 & 2 & $\ldots \ldots-$ & $\mathrm{I}$ & $-\cdots-$ & 5980. 264 & 8 & $\ldots . .-$ & $\mathrm{I}$ & $\ldots-$ \\
\hline 5868.50 & 1 & $\ldots \ldots$ & I & $-\ldots$ & 5981. 24 & 2 & & I & \\
\hline 5868. 97 & 2 & - n- & I & $-1-1$ & 5981. 41 & 1 & $\ldots$ & I & 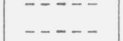 \\
\hline 5869.106 & 7 & $\ldots \ldots$ & $\mathrm{I}$ & $-1-1$ & 5983. 381 & 9 & 1 & I & $-1-1$ \\
\hline 5871.84 & 1 & $\ldots--$ & I & $\ldots-$ & 5986. 22 & 1 & $\ldots \ldots$ & $\mathrm{I}$ & $\ldots$ \\
\hline 5872.93 & $3 \mathrm{~h}$ & $\ldots \ldots$ & $\vec{I}$ & $\ldots$ & 5986.62 & 20 & 2 & $\mathrm{I}$ & 1 \\
\hline 5873.712 & 7 & $\ldots \ldots$ & I & $\ldots-\ldots$ & 5989. 76 & 2 & - . . - & I & $\ldots-\ldots$ \\
\hline 5879.772 & 8 & $\ldots \ldots$ & $\mathrm{I}$ & $\ldots \ldots$ & 5991.567 & 4 & -..... & $\mathrm{I}$ & $\ldots-\ldots$ \\
\hline 5881. 44 & 1 & ---- & I & $\ldots-$ & 5991. 806 & 5 & $\ldots \ldots$ & I & $\ldots-$ \\
\hline 5882.50 & 2 & $--\bar{x}--$ & I & $-\cdots-$ & 5991. 97 & 4 & $\ldots \ldots$ & I & 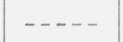 \\
\hline 5883.650 & 70 & 10 & I & 7 & 5992. 08 & 3 & $\ldots \ldots$ & I & $-\cdots--$ \\
\hline 5885.04 & 3 & ---- & I & --- & 5992. 97 & 50 & 6 & I & 7 \\
\hline 5886.31 & 30 & 2 & $\mathrm{I}$ & 3 & 5993. 07 & 5 & -...- & I & $\ldots$ \\
\hline 5887.43 & 20 & 2 & I & 1 & 5994.58 & 10 & $\ldots \ldots$ & I & 2 \\
\hline 5890.46 & 200 & 30 & I & 1 & 5996. 70 & 1 & $\ldots$. & I & $\ldots-$ \\
\hline 5891. 18 & 5 & $\ldots \ldots$ & I & $\ldots+$ & 5996. 99 & 1 & $\ldots-\cdots$ & I & 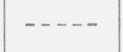 \\
\hline 5892.80 & 3 & & I & & 5998.05 & 3 & -..- & I & \\
\hline 5896. 63 & 50 & 4 & I & 7 & 6000.56 & 1 & - - - - & I & $-\ldots$ \\
\hline 5898.19 & 1 & $15 \mathrm{Hl}$ & II & 7 & 6002.30 & 1 & - & I & $\ldots$ \\
\hline 5900.53 & 3 & $-\cdot \bar{n}-\cdot$ & I & $\ldots-$ & 6002.51 & 1 & $\ldots \ldots$ & I & $\ldots$ \\
\hline 5902.95 & 500 & 70 & I & 3 & 6002.64 & 2 & $\ldots \ldots$ & $\vec{I}$ & $-\cdots$ \\
\hline 5906. 259 & 4 & $\ldots$ & I & $\ldots \ldots$ & 6004.18 & 30 & 2 & I & 3 \\
\hline 5906. 393 & 8 & $\ldots \ldots$ & I & $\ldots-$ & 6006. 375 & 2 & $6 \mathrm{hl}$ & II & 4 \\
\hline 5911.506 & 4 & $\ldots \ldots$ & I & $\ldots$ & 6007.03 & 3 & $\ldots$. & I & $\ldots \ldots$ \\
\hline 5913. 91 & 2 & $-\ldots-$ & I & ..... & 6011. 843 & 4 & $\ldots \ldots$ & I & $\ldots$. \\
\hline 5914.73 & 1 & $\ldots \ldots$ & I & 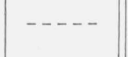 & 6014. 90 & 2 & $\ldots \ldots$ & $\mathrm{I}$ & $\ldots-\cdots$ \\
\hline 5918. 28 & 1 & & I & $\ldots$ & 6015. 360 & 10 & & I & \\
\hline 5922.94 & 10 & ----- & I & $\ldots--$ & 6016. 78 & 100 & 20 & I & 1 \\
\hline 5926.48 & 40 & 5 & I & 7 & 6017.83 & 2 & - - - - - & I & $\ldots$ \\
\hline 5927. 208 & 5 & $\ldots \ldots$ & I & $\ldots$ & 6018.46 & 2 & $-\ldots-$. & I & $\ldots$ \\
\hline 5927.53 & 2 & $\ldots$ & $\mathrm{I}$ & $\ldots$ & 6019.34 & 3 & - n & $\mathrm{I}$ & 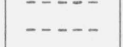 \\
\hline 5929. 36 & 2 & $15 \mathrm{hl}$ & II & 6 & 6021. 77 & 20 & 2 & I & 2 \\
\hline 5933.70 & 150 & 20 & I & 3 & 6022.54 & 1 & $\ldots-\ldots$ & $\hat{\mathrm{I}}$ & $\ldots-\ldots$ \\
\hline 5936. 02 & 5 & $\ldots-.-$ & I & $\ldots \ldots$ & 6024.93 & 2 & - - - - & $\vec{I}$ & 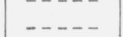 \\
\hline 5938.142 & 15 & 2 & I & 2 & 6026. 14 & 7 & $-\cdots---$ & I & ----- \\
\hline 5938.80 & 1 & $\ldots$ & I & $\cdots$ & 6027.559 & 5 & $200 \mathrm{~h}$ & II & 4 \\
\hline 5942.41 & 3 & $\ldots \ldots$ & I & $\ldots$ & 6030.58 & 3 & & $\mathrm{I}$ & \\
\hline 5944.10 & 1 & ...... & I & -.... & 6031. 92 & 2 & $20 \mathrm{hl}$ & II & 5 \\
\hline 5945.12 & 2 & $\ldots \ldots$ & I & $\cdots$ & 6033. 938 & 2 & $\ldots-\ldots$ & I & $\ldots--$ \\
\hline $\begin{array}{l}5945.78 \\
5946.524\end{array}$ & $\begin{array}{l}1 \\
7\end{array}$ & 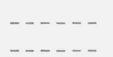 & $\begin{array}{l}\text { I } \\
\text { I }\end{array}$ & $\ldots$ & $\begin{array}{l}\text { 6038. } 82 \\
6039.711\end{array}$ & $\begin{array}{r}3 \\
10\end{array}$ & $-\cdots$ & $\begin{array}{l}\text { I } \\
\text { I }\end{array}$ & -... \\
\hline 5949. 390 & 6 & & $\mathrm{I}$ & & 6040.37 & 15 & 1 & I & 2 \\
\hline 5949.60 & 1 & - & $\mathrm{I}$ & $\ldots$ & 6041. 43 & 3 & $30 \mathrm{hl}$ & II & 5 \\
\hline 5949.80 & 2 & $\ldots \ldots$ & I & $\ldots$ & 6044.61 & 1 & & $\mathrm{I}$ & ---- \\
\hline 5950.26 & 1 & $\ldots$ & I & $\ldots-$ & 6047. 98 & 4 & $15 \mathrm{hl}$ & II & 5 \\
\hline 5951.24 & 1 & $\ldots--$ & I & $\ldots$. & 6048.86 & 1 & $-\ldots-.-$ & I & $\ldots .-$ \\
\hline 5952.86 & 2 & - . - & I & $\ldots-$ & 6052. 071 & 4 & & I & \\
\hline 5953.13 & 1 & $\ldots \ldots$ & I & $\ldots-$ & 6054. 166 & 80 & 6 & $\mathrm{I}$ & 7 \\
\hline 5954. 20 & $1 \mathrm{~h}$ & ---- & I & $\ldots-$ & 6059. 067 & 4 &.----- & $\mathrm{I}$ & $\ldots \ldots$ \\
\hline 5954. 71 & 4 & ---- & I & $\ldots$. & 6059.62 & 1 & $-\ldots-.-$ & I & $\ldots \ldots$ \\
\hline 5957.24 & 4 & $-\cdots-$ & I & $\ldots-$ & 6063.75 & 2 & $-\ldots-$. & $\mathrm{I}$ & $\ldots-$. \\
\hline 5959.30 & 2 & - - - & I & $\ldots$ & 6064. 257 & 10 & 1 & I & $-\ldots-$ \\
\hline 5959.57 & 5 & - - & I & $\ldots-$ & 6064. 712 & 4 & --.-- & I & -.... \\
\hline 5966. 30 & 4 & $\ldots \ldots$ & I & $\ldots$ & 6069. 20 & 3 & -...- & I & $\ldots$. \\
\hline $\begin{array}{l}5968.37 \\
5969.37\end{array}$ & $\begin{array}{l}1 \\
5\end{array}$ & $-\overline{20 h l}$ & $\begin{array}{ll}\text { I } & \\
& \text { II }\end{array}$ & $-\frac{--}{5}$ & $\begin{array}{l}6070.16 \\
6070.591\end{array}$ & $\begin{array}{l}1 \\
8\end{array}$ & 1 & $\begin{array}{l}\mathrm{I} \\
\mathrm{I}\end{array}$ & -... \\
\hline
\end{tabular}


TABLE 1. Emission spectra of hafuium-Continued

\begin{tabular}{|c|c|c|c|c|c|c|c|c|c|}
\hline \multirow[b]{2}{*}{$\begin{array}{c}\text { Wavelength } \\
\text { in air }\end{array}$} & \multicolumn{2}{|c|}{ Intensity } & \multirow[b]{2}{*}{ Spectrum } & \multirow[b]{2}{*}{$\begin{array}{c}\text { Zeeman } \\
\text { type }\end{array}$} & \multirow[b]{2}{*}{$\begin{array}{c}\text { Wavelength } \\
\text { in air }\end{array}$} & \multicolumn{2}{|c|}{ Intensity } & \multirow[b]{2}{*}{ Spectrum } & \multirow[b]{2}{*}{$\begin{array}{c}\text { Zeeman } \\
\text { type }\end{array}$} \\
\hline & Tube & Spark & & & & Tube & Spark & & \\
\hline$A$ & & & & & $A$ & & & & \\
\hline 6071.92 & 2 & - . - . - & I & $\ldots$ & 6168. 66 & 20 & 2 & I & 2 \\
\hline 6073.50 & 1 & - . - . & I & $\ldots . .$. & 6172.74 & 1 & $\ldots$ & I & $\ldots \ldots$ \\
\hline 6074. 360 & 10 & $\ldots \ldots$ & I & $\ldots-$ & 6173.89 & & $4 \mathrm{hl}$ & II & $-\ldots$ \\
\hline 6075.50 & 1 & $\ldots \ldots$ & I & $\ldots-$ & 6176.55 & 3 & $-\ldots-\ldots$ & I & $\ldots-$. \\
\hline 6077.53 & $2 \mathrm{~h}$ & $\ldots .$. & I & $\ldots-$ & 6176. 99 & 2 & $\ldots \ldots$ & I & \\
\hline 6079.59 & 1 & _. & I & $\ldots$ & 6185.128 & 400 & 40 & I & 3 \\
\hline 6084.67 & 1 & $\ldots \ldots$ & I & ---1 & 6189.21 & $\ldots$ & $8 \mathrm{Hl}$ & II & _- - - \\
\hline 6085. 71 & 3 & $\ldots \ldots$ & I & $\ldots \ldots$ & 6191. 33 & 1 & - - - - & I & $\ldots-\ldots$ \\
\hline 6090.80 & 4 & ----- & I & 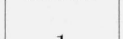 & 6191. 784 & 10 & $-\cdots--$ & I & $-\ldots-$ \\
\hline 6091.37 & 20 & 2 & I & 1 & 6192.475 & 50 & 5 & I & 2 \\
\hline 6092. 44 & 8 & - . - . & I & $\ldots-\ldots$ & 6193. 806 & 4 & & $\mathrm{I}$ & \\
\hline 6092.90 & 2 & & I & & 6198. 460 & 20 & 2 & $\mathrm{I}$ & 2 \\
\hline 6093. 14 & 2 & $20 \mathrm{hl}$ & II & 7 & 6200.890 & 4 & & $\mathrm{I}$ & $\ldots-\ldots$ \\
\hline 6094.88 & 4 & $\ldots-1-\bar{x}$ & I & $\ldots-\ldots$ & 6202.86 & 3 & 10 & II & 4 \\
\hline 6097.47 & $\ldots \ldots$ & $10 \mathrm{Hl}$ & II & 4 & 6206. 36 & 10 & $\ldots \ldots$ & I & $-\ldots$ \\
\hline 6098. 68 & 200 & 30 & I & 3 & 6206.51 & 12 & & I & \\
\hline 6104.58 & 20 & 3 & I & 2 & 6206. 926 & 1 & $30 \mathrm{hl}$ & II & 4 \\
\hline 6104.88 & 15 & 2 & I & $-\ldots$ & 6207. 08 & 1 & $-\ldots--$ & I & $-\ldots-$ \\
\hline 6105.07 & 3 & $\ldots \ldots$ & I & ---- & 6207. 46 & 10 & 1 & $\mathrm{I}$ & 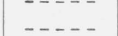 \\
\hline 6106.11 & 10 & 1 & I & $\ldots-$ & 6207.97 & 10 & 1 & I & $\ldots$. \\
\hline 6106. 22 & 2 & $\ldots \ldots$ & I & $\ldots-\ldots$ & 6209. 43 & 40 & 4 & I & 2 \\
\hline 6108.90 & 1 & - - - - & I & $\ldots$ & 6210.680 & 150 & 20 & $\overrightarrow{\mathrm{I}}$ & 2 \\
\hline 6109. 22 & 1 & $\ldots \ldots$ & I & $\ldots$ & 6211. 21 & 1 & $10 \mathrm{Hl}$ & II & $\ldots-\ldots$ \\
\hline 6115.29 & 3 & $-\ldots-$. & I & $\ldots$. & 6211. 60 & 4 & $\ldots-\ldots$ & I & $\ldots$ \\
\hline 6116.62 & 5 & $-\ldots$ & I & $-\ldots$ & 6211.70 & 3 & $\ldots$ & $\mathrm{I}$ & 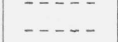 \\
\hline 6118. 16 & 40 & 6 & I & 1 & 6212.00 & 20 & 2 & I & 2 \\
\hline 6119.27 & 2 & - . . . . & I & $-\ldots$ & 6212.71 & 1 & & $\mathrm{I}$ & \\
\hline 6119.70 & 5 & - - & I & $\ldots$ & 6216.800 & 80 & 10 & $\overrightarrow{\mathrm{I}}$ & 7 \\
\hline 6122.00 & 15 & 2 & I & $\ldots . .$. & 6217.315 & 1 & 10 & II & $\ldots$ \\
\hline 6122.93 & 3 & $-\ldots--$ & I & $\ldots-$ & 6219.83 & 1 & ----- & I & $\ldots-\ldots$ \\
\hline 6126.30 & 6 & - . . - & I & - . - - & 6222.81 & 10 & $40 \mathrm{hl}$ & II & 6 \\
\hline 6128. 14 & 3 & - - - - & I & -.... & 6223. 74 & 1 & - & I & - . - - \\
\hline 6129.19 & 1 & --+- & I & $-\ldots$ & 6224.35 & 2 & - - - - - & $\mathrm{I}$ & $\ldots$ \\
\hline 6129.49 & 1 & $-1--$ & I & $\ldots$ & 6227. 03 & 2 & $\ldots--$ & $\mathrm{I}$ & $\ldots$ \\
\hline 6135.10 & 10 & $200 \mathrm{hl}$ & II & 4 & 6228. 20 & - . - & 2 & II & $\ldots .$. \\
\hline 6135. 40 & 10 & & I & -...- & 6229.10 & 15 & 1 & I & \\
\hline 6135.68 & 3 & 6 & II & $\ldots \ldots$ & 6229.63 & 1 & $20 \mathrm{hl}$ & II & $\ldots$ \\
\hline 6138.51 & 2 & - & I & -.... & 6230.825 & 6 & $60 \mathrm{hl}$ & II & 5 \\
\hline 6138.73 & 1 & $\ldots-\ldots$ & I & $\ldots$. & 6236.51 & 8 & 2 & I & $\ldots-$ \\
\hline 6139.00 & 3 & $\ldots \ldots$ & I & $\ldots$ & 6237.34 & 3 & ----- & I & - \\
\hline 6140.46 & 2 & - - - - & I & $\ldots$ & 6238.59 & 100 & 10 & I & 3 \\
\hline 6141.78 & 3 & - . - . - & I & $-1--$ & 6241.81 & 10 & 1 & $\mathrm{I}$ & 7 \\
\hline 6142.15 & 2 & $\ldots \ldots$ & I & $\ldots \ldots$ & 6242.27 & 7 & $\ldots-\ldots$ & $\mathrm{I}$ & $\ldots-\ldots$ \\
\hline 6142.77 & 1 & - . . & I & $\ldots \ldots$ & 6243.83 & 1 & & I & $\ldots$ \\
\hline 6144. 392 & 30 & 2 & I & 1 & 6248.94 & 50 & 200 & II & 5 \\
\hline 6144.58 & 20 & - - . - & I & $\ldots$ & 6250.16 & 1 & --- & I & $\ldots$ \\
\hline 6146.54 & 5 & $\ldots$ & I & $-\ldots$ & 6250.36 & 2 & _. & I & - \\
\hline 6146. 99 & 3 & - - - - & I & $-\ldots$ & 6252.92 & 1 & $\ldots$ & $\mathrm{I}$ & $-\ldots$ \\
\hline 6147. 24 & 3 & $\ldots \ldots$ & I & $\ldots \ldots$ & 6256.97 & 70 & 9 & I & 1 \\
\hline 6148. 68 & 7 & 1 & I & $-\ldots-$ & 6258.79 & 40 & 4 & I & 2 \\
\hline 6150.01 & 3 & $\ldots-$ & I & $-\ldots$ & 6262. 71 & 1 & ... & I & $\ldots$ \\
\hline 6152.952 & 10 & 1 & I & 1 & 6262.94 & 3 & - . . - & I & $\ldots$ \\
\hline 6156. 264 & 3 & $20 \mathrm{hl}$ & II & 5 & 6266.63 & 1 & $\ldots \ldots$ & I & $\ldots$. \\
\hline 6156.54 & $\ldots \ldots$ & 4hl & II & $\ldots$ & 6270.44 & 2 & $--\overline{1}-\overline{1}$ & $\vec{I}$ & $\ldots$. \\
\hline 6158.76 & $\ldots$ & $20 \mathrm{hl}$ & II & 6 & 6271.06 & 3 & $15 \mathrm{hl}$ & II & $-\ldots$ \\
\hline 6160.681 & $\ldots \ldots$ & $30 \mathrm{hl}$ & II & 6 & 6271.355 & 15 & 2 & I & $\ldots$ \\
\hline 6164.70 & 2 & - & I & $\ldots$ & 6272.770 & 9 & 1 & I & $-\ldots$ \\
\hline 6166.59 & 1 & -.... & I & $\ldots-\ldots$ & 6273.25 & 1 & $\ldots \ldots$ & I & $\ldots$ \\
\hline 6167.04 & 10 & $\ldots-\ldots$ & I & $\ldots-$ & 6275. 49 & 15 & $\ldots \ldots$ & I & $\ldots$ \\
\hline 6168.03 & 3 & - & $\mathrm{I}$ & $\ldots$ & 6276.41 & 3 & - - - - & $\mathrm{I}$ & $-1--$ \\
\hline
\end{tabular}


TABLE 1. Emission spectra of hafnium-Continued

\begin{tabular}{|c|c|c|c|c|c|c|c|c|c|}
\hline \multirow[b]{2}{*}{$\begin{array}{c}\text { Wavelength } \\
\text { in air }\end{array}$} & \multicolumn{2}{|c|}{ Intensity } & \multirow[b]{2}{*}{ Spectrum } & \multirow[b]{2}{*}{$\begin{array}{c}\text { Zeeman } \\
\text { type }\end{array}$} & \multirow[b]{2}{*}{$\begin{array}{c}\text { Wavelength } \\
\text { in air }\end{array}$} & \multicolumn{2}{|c|}{ Intensity } & \multirow[b]{2}{*}{ Spectrum } & \multirow[b]{2}{*}{$\begin{array}{c}\text { Zeeman } \\
\text { type }\end{array}$} \\
\hline & Tube & Spark & & & & Tube & Spark & & \\
\hline$A$ & & & & & $A$ & & & & \\
\hline 6277. 197 & 10 & 1 & I & 7 & 6376.19 & 20 & 2 & I & 3 \\
\hline 6278. 40 & 1 & $\ldots$ & I & $\ldots$ & 6376.88 & 1 & & I & $\ldots$ \\
\hline 6278. 804 & 4 & $\ldots-\ldots$ & I & $\ldots-\ldots$ & 6377.53 & 3 & & I & $-\cdots$ \\
\hline $\begin{array}{l}6279.834 \\
6290.10\end{array}$ & 15 & 60 & II & 6 & $\begin{array}{l}63806.19 \\
638\end{array}$ & $\begin{array}{l}80 \\
10\end{array}$ & 7 & I & 3 \\
\hline 0290.10 & & 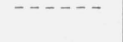 & 1 & $\cdots$ & 0281.504 & 10 & - - - - & & $\cdots$ \\
\hline 6296. 27 & 5 & $\ldots \ldots$ & I & - . . - & 6382. 232 & 10 & & I & \\
\hline 6297. 903 & 4 & $\ldots \ldots$ & I & -...- & 6383.69 & 20 & 1 & I & \\
\hline 6298. 12 & 3 & $\ldots--$ & I & $-\ldots$ & 6386. 229 & 300 & 40 & $\mathrm{I}$ & 2 \\
\hline 6298. 63 & 1 & $-\ldots--$ & I & $\ldots \ldots$ & 6388. 914 & 20 & 2 & $\mathrm{I}$ & 3 \\
\hline 6299.52 & 50 & 6 & I & 1 & 6390.22 & 10 & $\ldots$ & I & $\ldots$ \\
\hline 6299. 99 & 8 & - - - & I & - - - & 6390.40 & 2 & $\ldots$ & I & - - - - - \\
\hline 6300.128 & 20 & $\ldots \ldots$ & I & $\ldots$ & 6396.67 & 1 & $\ldots \ldots$ & I & $\ldots-$ \\
\hline 6300. 72 & 8 & $\ldots \ldots$ & I & -.- - & 6397. 91 & 1 & $-\ldots-\ldots$ & I & $\ldots$ \\
\hline 6300.94 & 2 & $-\ldots---$ & I & $\ldots$ & 6398. 94 & 1 & $\ldots \ldots$ & I & $\ldots-$ \\
\hline 6302.63 & 1 & - - - - & I & - - - - & 6400.24 & 2 & $\ldots \ldots$ & I & $\ldots$ \\
\hline 6303. 16 & 2 & $-\ldots-$ & I & - - - - & 6406.53 & 15 & & I & \\
\hline 6303. 90 & 1 & $\ldots \ldots$ & I & $\ldots$ & 6409.50 & 70 & 7 & I & $4 ?$ \\
\hline 6304.31 & 1 & $\ldots \ldots$ & I & $-\ldots-$ & 6418. 02 & 10 & $-\ldots--$ & $\mathrm{I}$ & 7 \\
\hline 6306.00 & 3 & $--\bar{x}$ & I & $\ldots--$ & 6420.58 & 1 & $\ldots \ldots$ & $\mathrm{I}$ & $\ldots$ \\
\hline 6306. 201 & 1 & $15 \mathrm{Hl}$ & II & 4 & 6420.86 & 1 & $\ldots \ldots$ & $\mathrm{I}$ & $\cdots$ \\
\hline 6306.50 & 2 & - - - - & I & - - - - & 6421.10 & 6 & - - - . & I & $\ldots-$ \\
\hline 6307. 91 & 1 & $\ldots-\ldots$ & I & - - - & 6421.91 & 2 & - . . . & I & $-\ldots$ \\
\hline 6309.67 & 1 & $\ldots \ldots$ & I & ----- & 6422.76 & 1 & -..... & $\bar{I}$ & $\ldots$ \\
\hline 6310.74 & 10 & 1 & I & $\ldots-\ldots$ & 6423. 40 & 5 & ---- & $\mathrm{I}$ & $\ldots$ \\
\hline 6311. 844 & 50 & 7 & I & 3 & 6429.44 & 10 & - . - - & I & 3 \\
\hline 6312.72 & 3 & ---- & I & - - - - & 6430.75 & 5 & $-\cdots--$ & I & -...- \\
\hline 6313. 41 & 30 & 2 & I & $--z--$ & 6431.46 & 1 & $-\cdots--$ & $\mathrm{I}$ & $\ldots$ \\
\hline 6315. 92 & 2 & $30 \mathrm{hl}$ & II & 5 & 6434.52 & 2 & $\ldots \ldots$ & I & $\ldots$ \\
\hline 6318. 313 & 30 & 3 & I & 1 & 6434. 77 & 4 & $\ldots \ldots$ & I & $\ldots$. \\
\hline 6323.69 & 1 & - . - & I & ---- & 6435.60 & 1 & $\ldots$ & I & $\ldots$ \\
\hline 6328.51 & 3 & - - - - & I & $\ldots-\ldots$ & 6436. 99 & 6 & $\ldots-\ldots$ & I & \\
\hline 6328. 76 & 1 & $\ldots--$ & I & - . - - & 6438.36 & 1 & - . - - & $\mathrm{I}$ & $\ldots$ \\
\hline 6330. 66 & 3 & ------ & I & - - - - & 6439.03 & 1 & $-\ldots \ldots$ & I & $\ldots$ \\
\hline 6337. 74 & ---- & 2 & II & ----- & 6442. 22 & 1 & $\ldots \ldots$ & I & $\ldots$ \\
\hline 6338. 096 & 80 & 9 & I & 1 & 6443.11 & 1 & - n... & I & $\ldots$ \\
\hline 6340. 24 & 3 & - - - & I & $-\ldots$ & 6444. 07 & 5 & 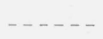 & I & - - \\
\hline 6340. 48 & 3 & ----- & I & $\ldots-$ & 6447.10 & 1 & $-\ldots$ & I & $\ldots$ \\
\hline 6340. 708 & 15 & 2 & I & - . - & 6449. 166 & 4 & $\ldots \ldots$ & I & $\ldots$ \\
\hline 6343. 742 & 1 & 8hl & II & $-\ldots$ & 6449.52 & 2 & -.... & I & $\ldots \ldots$ \\
\hline 6343.98 & 2 & $\cdots-\cdots$ & 1 & $-\cdots$ & 6451.16 & 1 & $-\cdots--$ & 1 & $\cdots$ \\
\hline 6345.08 & 3 & $-\cdots$ & I & -...- & 6454.466 & 3 & & $\mathrm{I}$ & $\ldots$ \\
\hline 6347.10 & 8 & $\ldots \ldots$ & I & $\ldots$ & 6455.84 & 2 & $15 \mathrm{hl}$ & II & $\ldots \ldots$ \\
\hline 6347.37 & 2 & 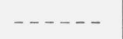 & I & -... & 6456.956 & 50 & 6 & $\mathrm{I}$ & 3 \\
\hline 6347. 63 & 5 & $\ldots \ldots$ & I & -...- & 6461.49 & 1 & $-\ldots-\ldots$ & I & $\ldots$ \\
\hline 6349.91 & 4 & - - - & I & $-\ldots$ & 6462.28 & 2 & 4 & II & 4 \\
\hline 6353. 46 & 3 & - - & I & $\ldots-$ & 6466.59 & 1 & $\ldots \ldots-$ & I & \\
\hline 6354.30 & 1 & $-\ldots--$ & I & -...- & 6467. 931 & 8 & $\ldots \ldots$ & $\vec{I}$ & $\ldots$ \\
\hline 6355. 393 & 5 & $\ldots \ldots$ & I & -...- & 6468. 32 & 4 & $\ldots \ldots$ & I & $\ldots \ldots$ \\
\hline 6355.68 & 4 & $-\ldots$ & I & $-\ldots$ & 6469.324 & 3 & - . - - & I & $\ldots$. \\
\hline 6355.99 & 1 & $-\cdots--$ & I & - - - & 6472.14 & 1 & $\ldots \ldots$ & I & $\ldots$. \\
\hline 6357. 47 & 1 & $-\ldots-$ & I & - - - - & 6472.67 & 6 & & I & \\
\hline 6359.84 & 10 & 1 & I & $-\ldots$ & 6473. 882 & 3 & 20hl & II & 4 \\
\hline 6363. 08 & 1 & ----- & I & $-\ldots$ & 6474.14 & 4 & $\ldots \ldots$ & I & $\ldots$. \\
\hline 6366. 87 & 1 & $\cdots+-$ & I & $-\cdots$ & 6475.04 & 1 & - . - - & I & $\ldots$ \\
\hline 6367. 09 & 3 & $-\cdots-\cdots$ & I & $-\cdots$ & 6477.03 & 4 & ...... & I & $\cdots$ \\
\hline 6370. 018 & 10 & 1 & I & $-\ldots$ & 6478. 72 & 1 & $\cdots \cdots$ & I & -...- \\
\hline 6371. 36 & 4 & - - & I & -...- & 6479.486 & 7 & - . - . - & I & $\ldots . .$. \\
\hline 6372. 792 & 2 & $\ldots \ldots$ & I & -...- & 6481.55 & 4 & - . - - & I & $-\ldots-$ \\
\hline 6373. 46 & 1 & $-\cdots-$ & I & $-\ldots$ & 6482. 526 & 3 &.----- & I & - . . \\
\hline 6375.03 & 1 & $-\cdots-$ & I & ---- & 6483.32 & 1 & $\ldots \ldots$ & I & $\ldots$ \\
\hline
\end{tabular}


TABLE 1. Emission spectra of hafnium-Continued

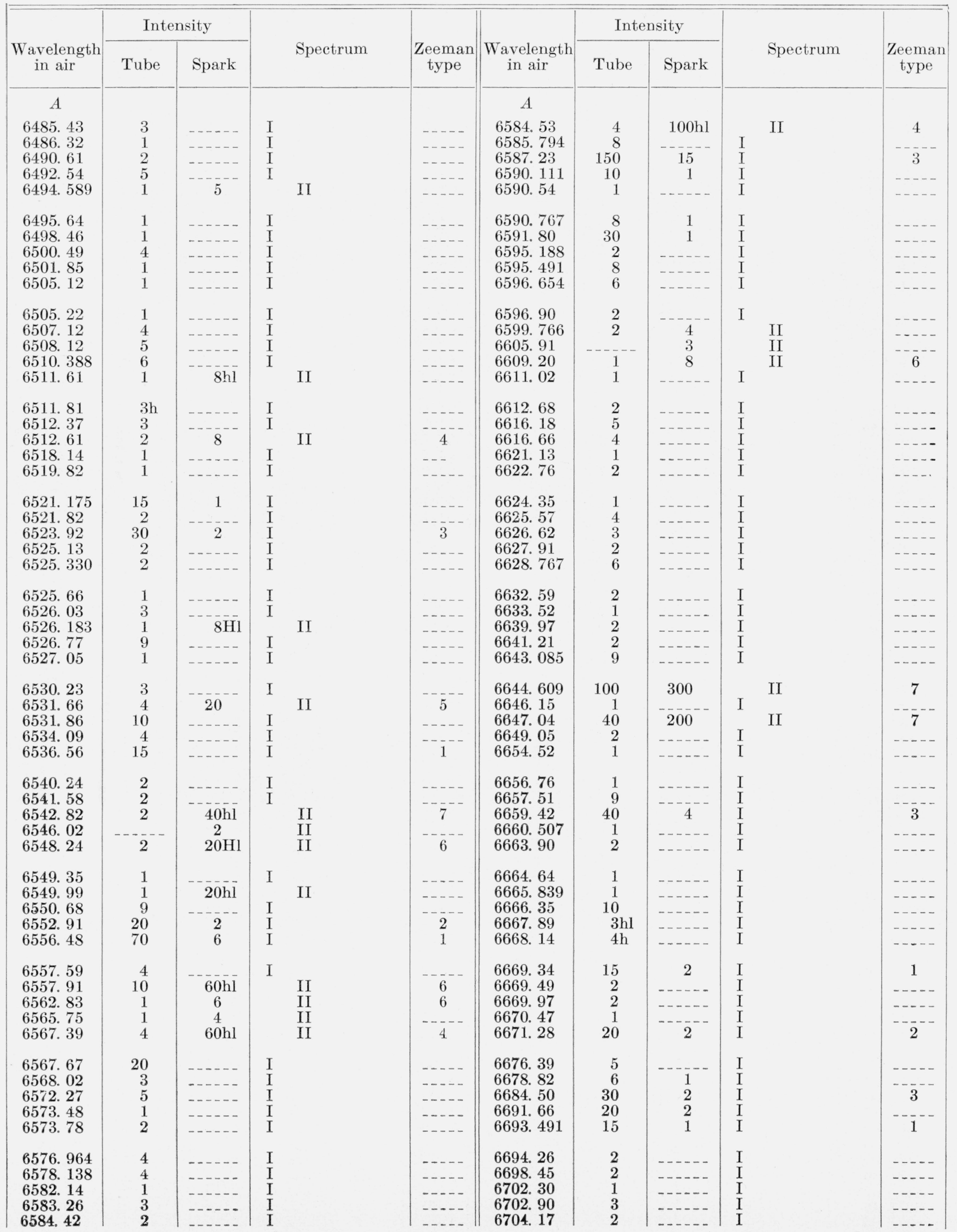


TABLE 1. Emission spectra of hafnium-Continued

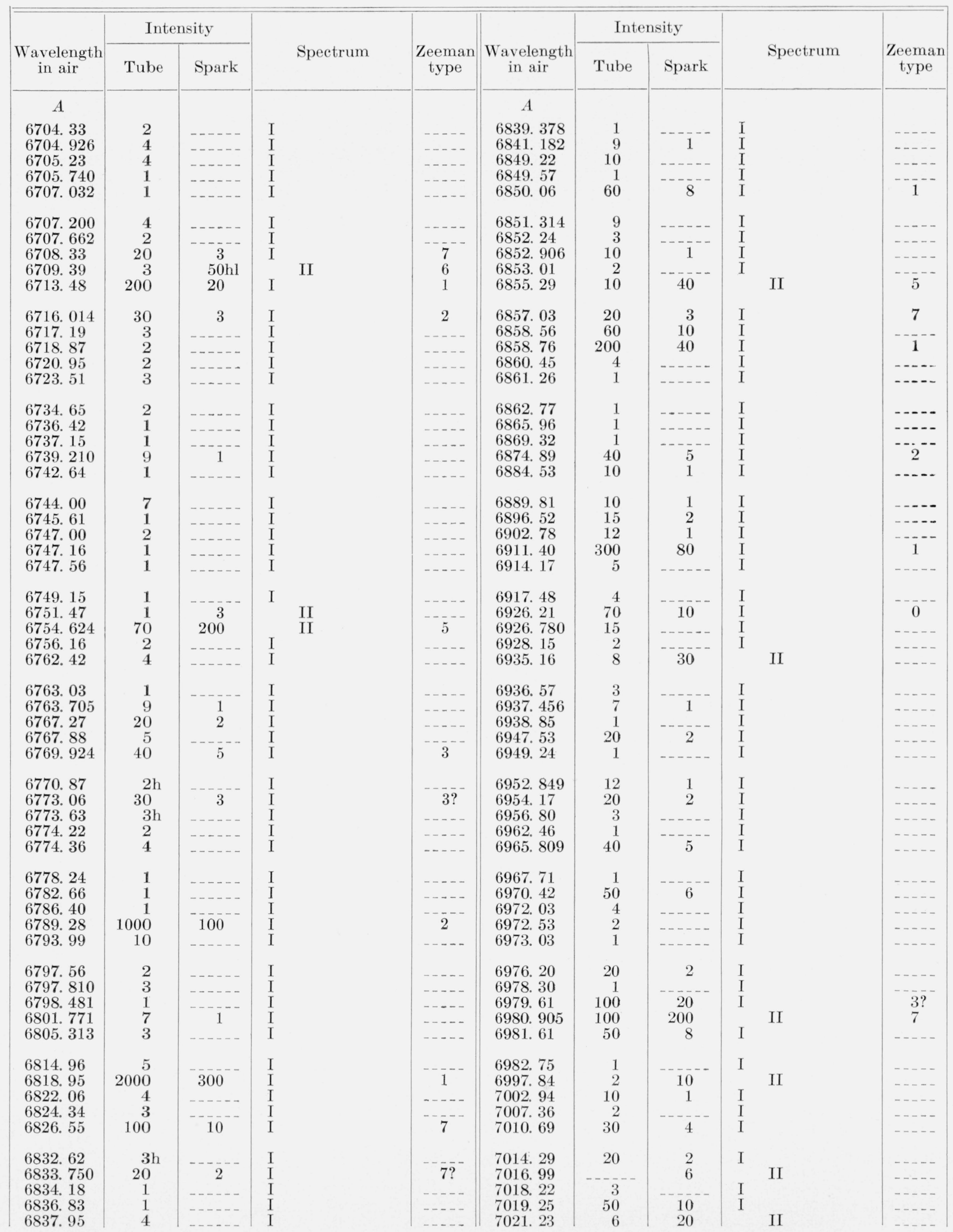


TABLE 1. Emission spectra of hafnium-Continued

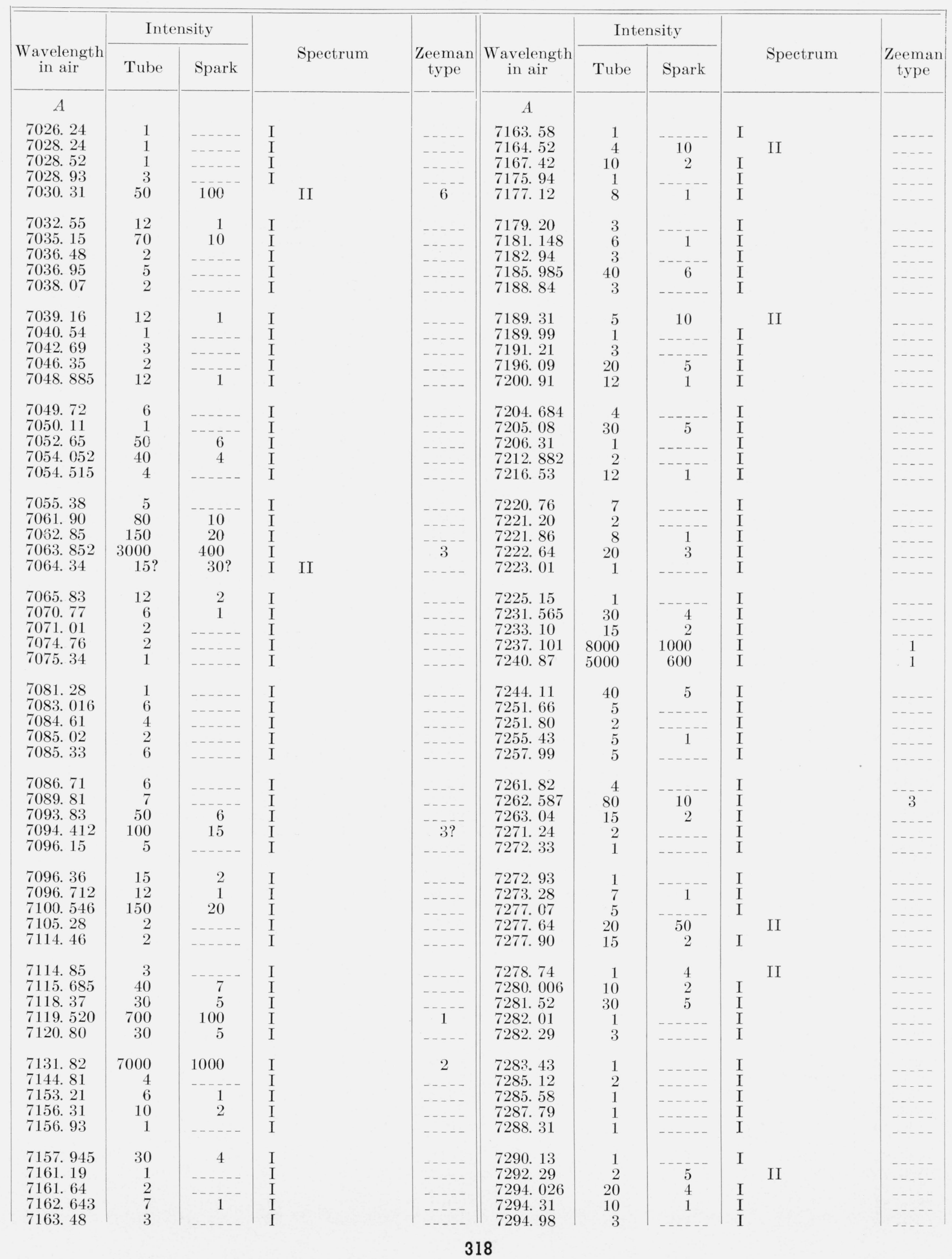


TABLE 1. Emission spectra of hafnium-Continued

\begin{tabular}{|c|c|c|c|c|c|c|c|c|c|c|}
\hline \multirow[b]{2}{*}{$\begin{array}{c}\text { Wavelength } \\
\text { in air }\end{array}$} & \multicolumn{2}{|c|}{ Intensity } & & \multirow[b]{2}{*}{ Spectrum } & \multirow[b]{2}{*}{$\begin{array}{c}\text { Zeeman } \\
\text { type }\end{array}$} & \multirow[b]{2}{*}{$\begin{array}{l}\text { Wavelength } \\
\text { in air }\end{array}$} & \multicolumn{2}{|c|}{ Intensity } & \multirow[b]{2}{*}{ Spectrum } & \multirow[b]{2}{*}{$\begin{array}{c}\text { Zeeman } \\
\text { type }\end{array}$} \\
\hline & Tube & Spark & & & & & Tube & Spark & & \\
\hline$A$ & & & & & & $A$ & & & & \\
\hline 7297. 06 & 1 & . . . - & I & & - . - & 7430.09 & 4 & & I & $\ldots--$ \\
\hline 7297. 33 & 2 & - . - & I & & $-\ldots$ & 7437. 600 & 500 & 100 & I & 3 \\
\hline 7298. 79 & 3 & - - - - & I & & $\therefore \ldots$ & 7441.16 & 6 & $\ldots \ldots$ & I & $\ldots \ldots$ \\
\hline 7301. 03 & 5 & 1 & I & & $\ldots$. & 7444. 26 & 20 & $3 ?$ & I & $\ldots$. \\
\hline 7303. 63 & 15 & 2 & I & & 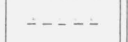 & 7444.81 & 12 & $1 ?$ & I & - . - \\
\hline 7309. 38 & 5 & & I & & & 7447. 19 & 3 & - . - & I & $\ldots$ \\
\hline 7312.68 & 1 & 2 & & II? & $-\ldots$ & 7447.39 & 1 & - & $\mathrm{I}$ & $\ldots \ldots$ \\
\hline 7315. 25 & 10 & 1 & I & & $\ldots$ & 7447.724 & 5 & $\ldots \ldots$ & I & $\ldots$. \\
\hline 7315. 72 & 1 & 2 & & II? & $\ldots$ & 7448. 66 & 7 & $\ldots--$ & I & $\ldots$ \\
\hline 7316. 94 & 2 & $--\ldots$ & I & & $\ldots$. & 7450.59 & 1 & $\ldots-\ldots$ & I & $\ldots$. \\
\hline 7320. 06 & 1000 & 200 & I & & 7 & 7451.94 & 6 & & I & .... \\
\hline 7321.79 & 150 & 30 & I & & 1 & 7461.30 & 10 & 1 & I & $\ldots \ldots$ \\
\hline 7322.91 & 15 & 2 & I & & $-\ldots$ & 7462.51 & 3 & & I & $-\cdots=-$ \\
\hline 7323. 43 & $3 \mathrm{~h}$ & $\ldots-\ldots$ & I & & $\ldots$ & 7463.905 & 200 & 40 & I & 7 \\
\hline 7324. 13 & 2 & $\ldots \ldots$ & I & & $\therefore-$ & 7473.67 & 6 & $\ldots \ldots$ & I & $\ldots \ldots$ \\
\hline 7327.175 & 15 & 2 & I & & $\ldots \ldots$ & 7476.53 & 2 & $\ldots$ & I & $\ldots \ldots$ \\
\hline 7328. 63 & 15 & 30 & & II & $-\ldots$ & 7476.71 & 4 & $\ldots \ldots$ & I & $\ldots$. \\
\hline 7334.73 & 1 & & I & & $\ldots$ & 7479.78 & 15 & 2 & I & $\ldots$. \\
\hline 7334.88 & 2 & 3 & $\mathrm{I}$ & II? & $\ldots$ & 7480.781 & 20 & 2 & I & $\ldots$ \\
\hline 7337. 93 & 12 & 2 & I & & $\ldots$ & 7483.704 & 4 & $\ldots \ldots$ & I & $\ldots$. \\
\hline 7339. 294 & 40 & 7 & $\mathrm{I}$ & & & 7484.603 & 50 & 10 & I & $\ldots$ \\
\hline 7342.01 & 1 & $\ldots$ & $\mathrm{I}$ & & $\ldots$ & 7488.17 & 30 & 4 & $\mathrm{I}$ & $\ldots$ \\
\hline 7342.80 & 1 & - & I & & $-\ldots$ & 7489.02 & 15 & 2 & I & $\ldots$. \\
\hline 7344. 70 & 2 & - & I & & $\ldots$ & 7492.11 & 10 & 1 & I & $\ldots$ \\
\hline 7346. 14 & $3 \mathrm{~h}$ & $\ldots \ldots$ & I & & $\ldots$ & 7501.63 & 1 & $\ldots$ & I & $\ldots$. \\
\hline 7352. 41 & 1 & & I & & - - - & 7506.80 & 3 & $\ldots$ & I & $\ldots \ldots$ \\
\hline 7356. 18 & 100 & 15 & I & & $\ldots \ldots$ & 7508.77 & 2 & $\ldots \ldots$ & I & $\ldots \ldots$ \\
\hline 7358. 78 & 3 & $\ldots-\ldots$ & I & & $-\ldots$ & 7511.00 & $10 ?$ & $\ldots-\ldots$ & I & $\ldots \ldots$ \\
\hline 7359. 916 & 3 & $\ldots \ldots$ & I & & $\ldots$ & 7511.47 & 1 & $\ldots \ldots$ & I & $\ldots$. \\
\hline 7365.35 & 100 & 20 & I & & $\ldots$ & 7513.36 & 2 & $\ldots \ldots$ & I & $\therefore \ldots$ \\
\hline 7368. 160 & 30 & 6 & I & & $\ldots \ldots$ & 7514. 98 & 3 & 5 & II & $\ldots$ \\
\hline 7374.389 & 15 & 2 & I & & - . . & 7515. 22 & 3 & $\ldots \ldots$ & I & $\ldots$. \\
\hline 7375.59 & 1 & $\ldots-\ldots$ & $\mathrm{I}$ & & $\ldots \ldots$ & 7516.79 & 2 & $\ldots \ldots$ & I & $\ldots \ldots$ \\
\hline 7376. 987 & 4 & $\ldots \ldots$ & I & & $-\ldots$ & 7517. 49 & 1 & $\ldots \ldots$ & I & $\ldots$. \\
\hline 7377.176 & 8 & 1 & I & & $\ldots$. & 7520.38 & 3 & - . . - & I & $\ldots$ \\
\hline 7378. 91 & 2 & $\ldots-\cdots$ & I & & & 7525. 09 & 2 & & I & $\ldots$ \\
\hline 7381.77 & 1 & - & I & & $\ldots$ & 7526.82 & 15 & 2 & $\mathrm{I}$ & $\ldots$ \\
\hline 7384. 03 & 10 & - & I & & $\ldots$ & 7529. 21 & 2 & $\ldots \ldots$ & I & $\ldots . .$. \\
\hline 7384.75 & 1 & $\ldots$ & I & & $\ldots$ & 7530.13 & $5 \mathrm{~h}$ & $\ldots \ldots$ & I & $\ldots .$. \\
\hline 7385.35 & 3 & $\ldots-\ldots$ & $\overline{\mathrm{I}}$ & & $\ldots$ & 7532.42 & 1 & $\ldots \ldots$ & I & $\ldots$. \\
\hline 7388. 86 & 4 & & I & & & 7532.88 & 5 & $\ldots$ & I & $\ldots$. \\
\hline 7390. 729 & 400 & 60 & I & & 3 & 7534.81 & 1 & $\ldots \ldots$ & I & $\ldots$. \\
\hline 7392.86 & 4 & $\ldots$ & $\mathrm{I}$ & & $\ldots$ & 7535. 11 & 2 & $\ldots \ldots$ & I & $\ldots .$. \\
\hline 7396. 99 & 15 & 2 & I & & $\ldots$ & 7537. 35 & 3 & $\ldots \ldots$ & I & $\ldots$. \\
\hline 7398. 137 & 12 & 2 & I & & $\ldots$ & 7542.39 & 10 & 1 & I & $\ldots .$. \\
\hline 7398. 97 & 3 & & I & & $\ldots \ldots$ & 7543. 40 & 50 & 6 & I & $\ldots$. \\
\hline 7400.72 & 12 & 2 & I & & $\ldots \ldots$ & 7544.81 & 3 & $\ldots \ldots$ & I & $\ldots$. \\
\hline 7402.75 & 15 & 1 & I & & $\ldots \ldots$ & 7551. 64 & 7 & 1 & I & $\ldots$ \\
\hline 7408.97 & 30 & 6 & $\mathrm{I}$ & & $\ldots$ & 7556.37 & 200 & 40 & I & 2 \\
\hline 7409.505 & 70 & 12 & I & & $\ldots$ & 7557.78 & 1 & $\ldots \ldots$ & I & $\ldots$. \\
\hline 7412.70 & 5 & & I & & & 7558.63 & 1 & & I & $\ldots$ \\
\hline 7414.63 & 20 & 2 & I & & $\ldots$ & 7561. 12 & 1 & 10 & II & $\ldots$ \\
\hline 7417.51 & 8 & 1 & I & & $\ldots$ & 7562.93 & 1500 & 300 & I & 1 \\
\hline 7420.48 & 3 & $\ldots \ldots$ & I & & $\ldots$ & 7564. 24 & 200 & 40 & $\mathrm{I}$ & 7 \\
\hline 7420.76 & 1 & $\ldots \ldots$ & I & & $-\ldots--$ & 7565.35 & $\ldots \ldots$ & 2 & II & $\ldots$ \\
\hline 7422. 416 & 15 & 2 & I & & $-\ldots-$ & 7568.85 & 2 & & I & 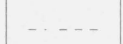 \\
\hline 7423. 628 & 120 & 20 & I & & 3 & 7570.90 & 1 & 2 & II & ..... \\
\hline 7424. 274 & 10 & $-\ldots-\ldots$ & $\mathrm{I}$ & & $\ldots$ & 7576.67 & 40 & 6 & I & $\ldots$ \\
\hline 7426.67 & 3 & $\ldots \ldots$ & I & & $\ldots$ & 7577.02 & 300 & 40 & $\mathrm{I}$ & 1 \\
\hline 7427.77 & 1 & 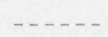 & I & & $\ldots$ & 7581.18 & 4 & $\ldots \ldots$ & I & $\ldots .$. \\
\hline
\end{tabular}


TABLE 1. Emission spectra of hafnium-Continued

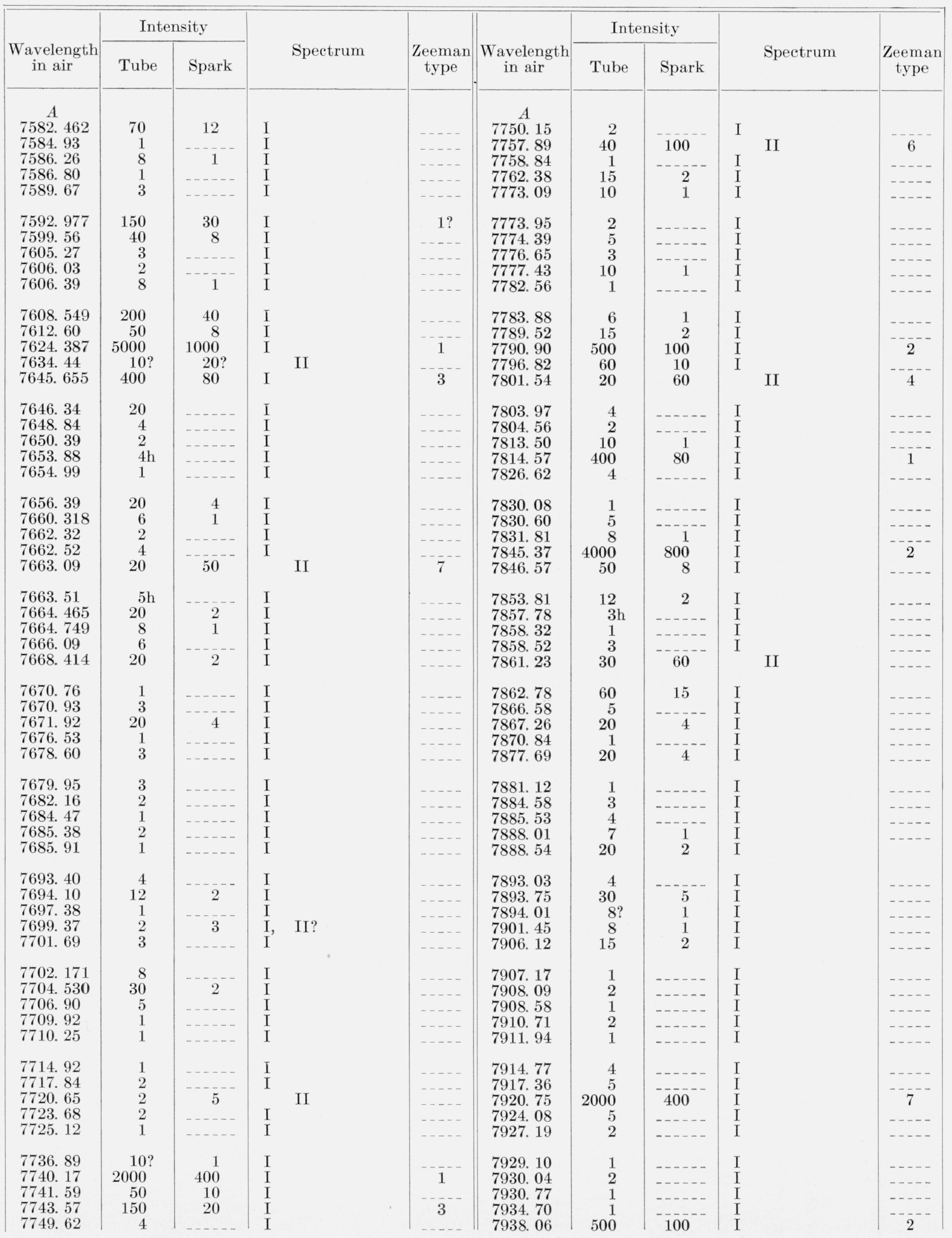


TABLE 1. Emission spectra of hafnium-Continued

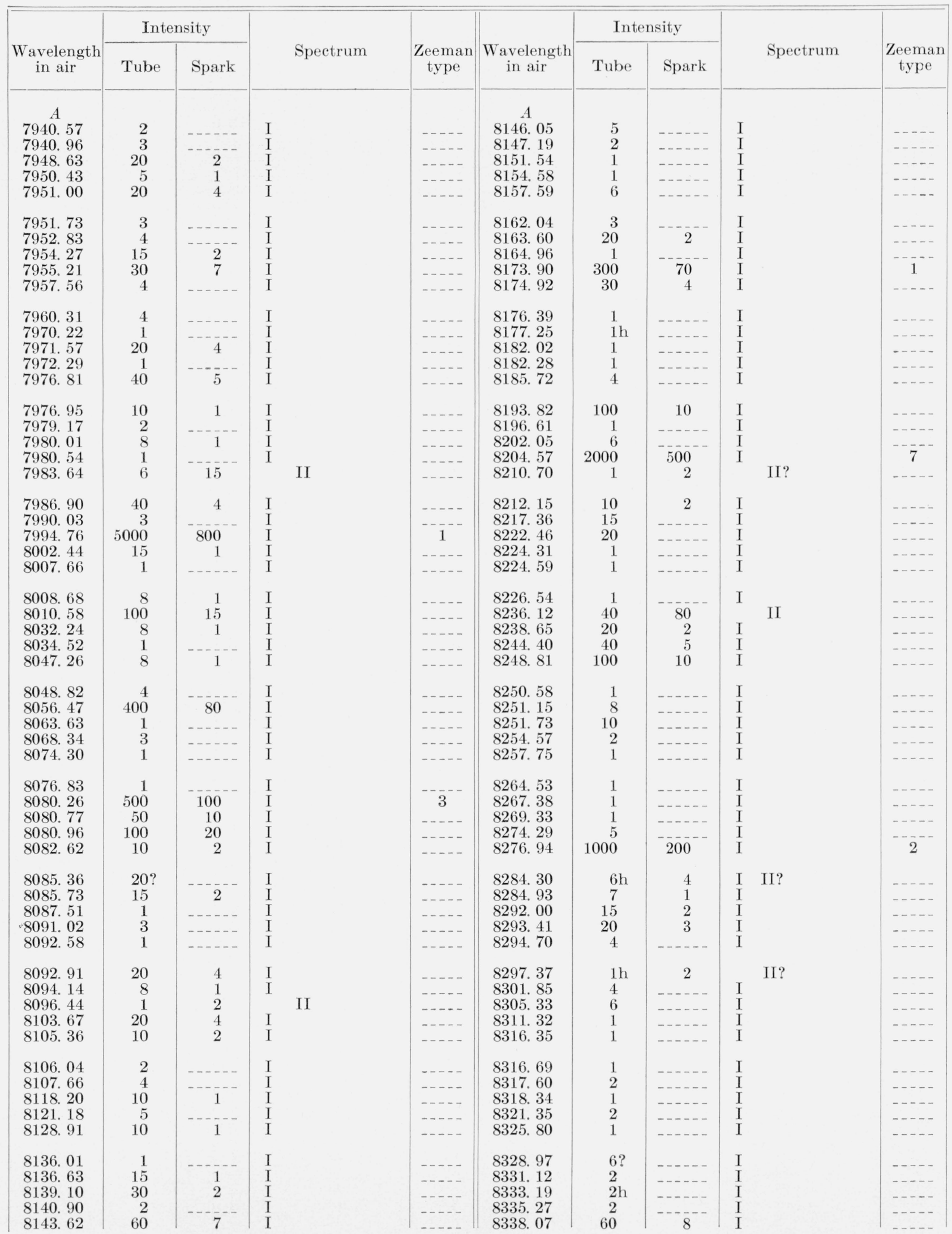


TABLE 1. Emission spectra of hafnium-Continued

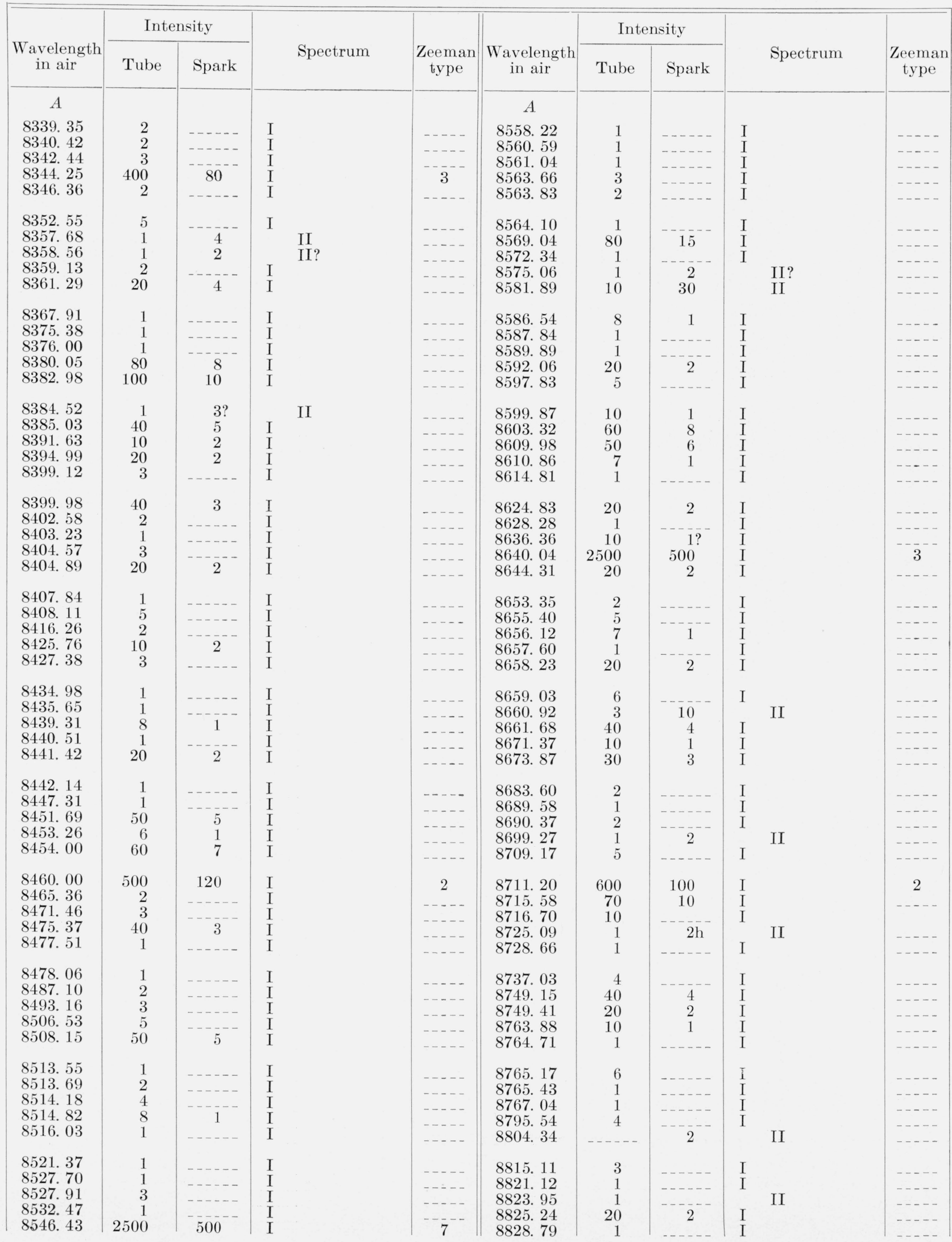


TABLE 1. Emission spectra of hafnium-Continued

\begin{tabular}{|c|c|c|c|c|c|c|c|c|c|}
\hline \multirow[b]{2}{*}{$\begin{array}{l}\text { Wavelength } \\
\text { in air }\end{array}$} & \multicolumn{2}{|c|}{ Intensity } & \multirow[b]{2}{*}{ Spectrum } & \multirow[b]{2}{*}{$\begin{array}{c}\text { Zeeman } \\
\text { type }\end{array}$} & \multirow[b]{2}{*}{$\begin{array}{c}\text { Wavelength } \\
\text { in air }\end{array}$} & \multicolumn{2}{|c|}{ Intensity } & \multirow[b]{2}{*}{ Spectrum } & \multirow[b]{2}{*}{$\begin{array}{l}\text { Zeeman } \\
\text { type }\end{array}$} \\
\hline & Tube & Spark & & & & Tube & Spark & & \\
\hline A & & & & & $A$ & & & & \\
\hline 8841.99 & 20 & 2 & I & $\ldots$ & 9482. 83 & 10 & 1 & I & $\ldots$ \\
\hline $\begin{array}{l}8845.95 \\
8871.65\end{array}$ & 5 & -...- & I & $\cdots$ & $\begin{array}{l}9483.52 \\
9493.42\end{array}$ & 1 & -- & I & $\cdots$ \\
\hline 8875.92 & 10 & - n-n & I & - & $\begin{array}{l}9493.42 \\
9498.81\end{array}$ & $\begin{array}{l}1 \\
30\end{array}$ & 3 & $\begin{array}{l}1 \\
\text { I }\end{array}$ & $\ldots$ \\
\hline 8876.17 & 5 & $\ldots$ & I & $\ldots$ & 9506.10 & 2 & & I & $\cdots$ \\
\hline 8886. 72 & 3 & - & I & -...- & 9513. 14 & 600 & 100 & I & - \\
\hline 8888. 43 & 1 & $\ldots$ & I & $\ldots$ & 9514. 37 & 8 & 3 & I & $\cdots$ \\
\hline 8902. 06 & 4 & $\ldots \ldots$ & I & $\ldots-$ & 9524.87 & 1 & & I & $\cdots-$ \\
\hline 8906. 81 & 1 & -.... & I & 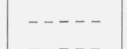 & 9553. 63 & 1 & & I & $\cdots-$ \\
\hline 8918. 06 & 2 & $\ldots-$ & I & $\cdots$ & 9563.26 & 200 & 50 & 1 & $\ldots$ \\
\hline 8927. 24 & 3 & -..... & I & $\ldots$ & 9566. 01 & 1 & & I & $\ldots$ \\
\hline 8952. 14 & 5 & $\ldots$ & I & $\ldots$. & 9581. 37 & 10 & 3 & I & \\
\hline 8958. 89 & 20 & $\ldots \ldots$ & I & 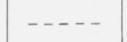 & 9587. 35 & 5 & -... & I & $\cdots-$ \\
\hline 8967.15 & 10 & $\ldots \ldots$ & I & $\ldots$ & 9613.15 & 3 & & I & $\ldots$ \\
\hline 8976.65 & 20 & -...- & I & 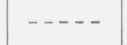 & 9616.05 & 5 & 2 & I & $\cdots \cdots$ \\
\hline 8994. 77 & 30 & 2 & I & ..... & 9628.91 & 1 & & I & ..... \\
\hline 9004.74 & 200 & 20 & I & $\ldots$ & 9652.05 & 50 & 7 & I & $\ldots$ \\
\hline 9031. 77 & 2 & & I & -...- & 9654.41 & 3 & & I & .... \\
\hline 9035.66 & 1 & $\ldots-$ & I & $\ldots$ & 9662. 40 & 1 & -2 & I & $\ldots$ \\
\hline 9052.35 & 10 & 3 & I & $\ldots$ & 9673.88 & 10 & 2 & I & ... \\
\hline 9093.88 & 2 & & I & - . - - & 9697.66 & 15 & 3 & I & $\ldots$ \\
\hline 9138. 20 & 30 & 3 & I & $\ldots$ & 9716.80 & 3 & & I & \\
\hline 9145.58 & 3 & & I & $\ldots$. & 9722,30 & 4 & 1 & I & $\ldots$ \\
\hline 9153. 00 & 2 & 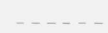 & I & $\ldots$ & 9737.93 & 4 & 1 & I & $\ldots \ldots$ \\
\hline 9158.55 & 9 & $\cdots$ & I & $-\ldots$ & 9746.45 & 2 & - & I & $\ldots$ \\
\hline 9177. 23 & 100 & 8 & I & $\ldots \ldots$ & 9752.40 & 20 & 10 & I & $\ldots \ldots$ \\
\hline 9179.30 & 4 & & I & $\ldots$ & 9754.43 & 1 & & I & $\ldots$. \\
\hline 9182.14 & 250 & 40 & I & $\ldots$ & 9791. 20 & 1 & & I & $\ldots$ \\
\hline 9193. 30 & 300 & 30 & I & $\ldots$ & 9793. 40 & 1 & & I & $\ldots$ \\
\hline 9216.86 & 10 & $\ldots \ldots$ & I & - . . - & 9801. 09 & 10 & 4 & I & $\ldots$ \\
\hline 9243. 18 & 1 & & I & - & 9805. 71 & 2 & & I & \\
\hline 9250.25 & 1000 & 150 & I & $\ldots$ & 9828. 22 & 15 & 4 & I & $\ldots \ldots$ \\
\hline 9282. 54 & 4 & $\ldots$ & I & $\ldots$ & 9864. 37 & 1 & & I & $\ldots \ldots$ \\
\hline 9295. 63 & 15 & 1 & I & $\ldots$ & 9877. 96 & 1 & $\ldots$ & I & $\ldots$ \\
\hline 9299. 95 & 60 & 5 & I & $\ldots$ & 10023. 30 & 2 & $\cdots$ & I & $\ldots$ \\
\hline 9306. 16 & 2 & $\ldots$ & I & $\ldots \ldots$ & 10036. 53 & 150 & 40 & I & $\ldots \ldots$ \\
\hline 9306. 79 & 1 & $\ldots$ & I & $\ldots$ & 10088. 51 & 40 & 8 & I & $\ldots$ \\
\hline 9308. 29 & 9 & $\cdots$ & I & $\ldots$ & 10103. 88 & 15 & 3 & I & $\ldots$ \\
\hline 9313. 65 & 2 & $\ldots \ldots$ & I & $\ldots$ & 10112. 20 & 10 & 2 & I & $\ldots$ \\
\hline 9314. 46 & 2 & $\ldots-$ & I & .... & 10120.15 & 3 & & I & - - - \\
\hline 9325. 14 & 80 & 10 & I & & 10135. 30 & 6 & 3 & I & \\
\hline 9341. 17 & 3 & $\ldots$ & I & $\ldots$ & 10168. 81 & 20 & 4 & I & $\ldots$ \\
\hline 9342. 00 & 2 & & I & $\ldots$ & 10181. 45 & 2 & $\ldots \ldots$ & I & $\ldots$ \\
\hline 9346. 32 & 150 & 20 & I & . & 10184.18 & 1 & $\ldots$ & I & $\ldots \ldots$ \\
\hline 9359. 86 & 1 & 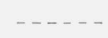 & I & -.... & 10190.70 & 2 & $\ldots \ldots$ & I & $\ldots$ \\
\hline 9365. 75 & 3 & $\ldots$ & I & $\ldots$ & 10203. 90 & 2 & & I & $\ldots$ \\
\hline 9372. 00 & 1 & $\ldots$ & I & $\ldots$ & 10207. 78 & 8 & 1 & I & $\ldots$ \\
\hline 9380. 14 & 50 & 7 & I & -.... & 10208. 74 & 2 & 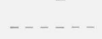 & I & $\ldots$ \\
\hline 9387.54 & 3 & & I & $\ldots$ & 10209.56 & 1 & 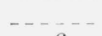 & I & - . - \\
\hline 9387. 94 & 2 & $\ldots \ldots$ & I & $\ldots$ & 10227. 18 & 20 & 6 & I & $\ldots$ \\
\hline 9389. 91 & 1 & $\ldots$ & I & $\ldots \ldots$ & 10259. 15 & 30 & 6 & I & $\ldots$ \\
\hline 9402.57 & 50 & 4 & I & & 10259.67 & 10 & & I & $\ldots$ \\
\hline 9404.14 & 4 & 2 & I II & $\ldots \ldots$ & 10293. 33 & 1 & & I & 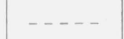 \\
\hline 9405.18 & 1 & & I & $\ldots$ & 10312. 19 & 1 & $\ldots$ & I & $\ldots$ \\
\hline 9412.23 & 2 & $\cdots$ & I & $\ldots$ & 10319.65 & 2 & $\ldots$. & I & $\ldots-$ \\
\hline 9453.75 & 12 & 2 & I & $\ldots \ldots$ & 10342. 55 & 3 & $\ldots \ldots$ & I & $\ldots$ \\
\hline 9488. 25 & 12 & 2 & I & $\ldots$ & 10361. 40 & 2 & & I & $\ldots$ \\
\hline 9469.05 & 1 & & I & ..... & 10377. 83 & 2 & $\ldots$ & I & -...- \\
\hline 9472.01 & 1 & $\ldots \ldots$ & I & $\ldots \ldots$ & 10388. 31 & 8 & $\ldots$ & I & $\ldots$ \\
\hline 9474.26 & 1 & & 1 & $\ldots$ & 10389.01 & 1 & & $\mathrm{I}$ & $\ldots . .-$ \\
\hline
\end{tabular}


TABle 1. Emission spectra of hafnium-Continued

\begin{tabular}{|c|c|c|c|c|c|c|c|c|c|}
\hline \multirow{2}{*}{$\begin{array}{l}\text { Wavelength } \\
\text { in air }\end{array}$} & \multicolumn{2}{|c|}{ Intensity } & \multirow{2}{*}{ Spectrum } & \multirow{2}{*}{$\begin{array}{c}\text { Zeeman } \\
\text { type }\end{array}$} & \multirow{2}{*}{$\begin{array}{l}\text { Wavelength } \\
\text { in air }\end{array}$} & \multicolumn{2}{|c|}{ Intensity } & \multirow{2}{*}{ Spectrum } & \multirow{2}{*}{$\begin{array}{c}\text { Zeeman } \\
\text { type }\end{array}$} \\
\hline & Tube & Spark & & & & Tube & Spark & & \\
\hline 10397. 45 & 200 & 50 & $\mathrm{I}$ & 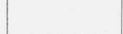 & $10850-40$ & 1 & & & \\
\hline 10405. 51 & 5 & $\ldots 0$ & I & - n- & $\begin{array}{l}10859.40 \\
10868.44\end{array}$ & 20 & 2 & I & $\ldots$ \\
\hline 10414. 09 & 3 & - n- & I & - n & 10876.28 & 1 & 2 & I & $\ldots$ \\
\hline 10418. 99 & 6 & 1 & $\mathrm{I}$ & $\ldots$ & 10882.85 & 1 & & $\mathrm{I}$ & $\ldots$ \\
\hline 10423. 78 & 400 & 150 & $\mathrm{I}$ & 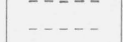 & 10900.75 & 15 & 5 & $\mathrm{I}$ & $\ldots$ \\
\hline 10438.63 & 2 & & $\mathrm{I}$ & $\ldots \ldots$ & 10914. 47 & 50 & 10 & I & $\ldots \ldots$ \\
\hline 10445. 17 & 7 & $\ldots \ldots$ & $\mathrm{I}$ & $\ldots$ & 10923. 38 & 2 & $\ldots+\ldots$ & I & $\ldots-$ \\
\hline 10457. 92 & 1 & $\ldots \ldots$ & $\mathrm{I}$ & $\ldots$ & 10933. 82 & 10 & $\ldots \ldots$ & $\overrightarrow{\mathrm{I}}$ & $\ldots$ \\
\hline 10472.68 & 1 & $\ldots \ldots$ & $\mathrm{I}$ & $\ldots$ & 10938.08 & 2 & $\ldots \ldots$ & I & $\ldots \ldots$ \\
\hline 10479.20 & 1 & $\ldots \ldots$ & I & $\ldots$ & 10953. 12 & 4 & $\ldots \ldots$ & I & $-\ldots-$ \\
\hline 10480. 21 & 200 & 30 & I & $\ldots$ & 10971. 61 & 50 & 5 & I & $\ldots \ldots$ \\
\hline 10484.44 & 3 & $\ldots \ldots$ & I & $\ldots \ldots$ & 11000.00 & 3 & & I & - - - \\
\hline 10496. 77 & 3 & $\ldots \ldots$ & I & $\ldots$ & 11030.13 & 20 & 1 & I & $\ldots$. \\
\hline 10499. 16 & 1 & $\ldots \ldots$ & $\mathrm{I}$ & $\ldots$ & 11039.01 & 4 & & I & $\ldots$ \\
\hline 10517. 74 & 1 & $\ldots \ldots$ & I & $\ldots$. & 11046. 97 & 40 & 4 & I & $\ldots$ \\
\hline 10527. 28 & 25 & 3 & I & $\ldots .$. & 11074. 42 & 5 & $\ldots$ & I & $\ldots \ldots$ \\
\hline 10529.38 & 2 & $\ldots \ldots$ & I & $\ldots$ & 11092.58 & 1 & $\ldots \ldots$ & I & $\ldots \ldots$ \\
\hline 10545.50 & 7 & 1 & I & & 11139.05 & 20 & . . . . & I & $\ldots \ldots$ \\
\hline 10547.44 & 1 & $\ldots$ & I & $\ldots \ldots$ & 11190.17 & 1 & $\ldots \ldots$ & I & ..... \\
\hline 10550.85 & 100 & 7 & $\mathrm{I}$ & $-\ldots$ & 11219.28 & 1 & & I & $\ldots$ \\
\hline 10588. 44 & 1 & & I & $\ldots \ldots$ & 11227.70 & 10 & & $\mathrm{I}$ & $\ldots \ldots$ \\
\hline 10594. 89 & 50 & 5 & $\mathrm{I}$ & $\ldots$ & 11229.01 & 15 & 1 & I & $\ldots$ \\
\hline 10622.14 & 12 & 1 & $\mathrm{I}$ & $\ldots$ & 11242.16 & 3 & $\ldots \ldots$ & I & $\ldots-\ldots$ \\
\hline 10634.81 & 5 & & $\mathrm{I}$ & $\ldots$ & 11296. 74 & 1 & - - - - - & I & $\ldots-\ldots$ \\
\hline 10637. 93 & 150 & 30 & $\mathrm{I}$ & $\ldots$. & 11303.44 & 10 & & I & $\ldots-$ \\
\hline 10642. 30 & 1 & & I & & 11365.02 & 25 & 7 & I & $\ldots \ldots$ \\
\hline 10661. 35 & 2 & - & $\mathrm{I}$ & $\ldots$ & 11393. 12 & 1 & & $\mathrm{I}$ & $\ldots$ \\
\hline 10698. 21 & 1 & - $\ldots$ & $\mathrm{I}$ & $-\cdots$ & 11480.61 & 15 & 2 & I & $-\ldots$ \\
\hline 10755.89 & 1 & $\ldots \ldots$ & $\mathrm{I}$ & $\ldots$ & 11602.16 & 4 & $\ldots \ldots$ & $\mathrm{I}$ & $\ldots \ldots$ \\
\hline 10756.97 & 1 & $\ldots \ldots$ & I & -...- & 11766.95 & 10 & -...- & I & $\ldots \ldots$ \\
\hline 10762.68 & 3 & & I & & 11960.80 & 2 & & I & $\ldots-$ \\
\hline 10772. 33 & 15 & 2 & I & $\ldots \ldots$ & 12043.08 & 6 & _. $\ldots$ & I & $\ldots \ldots$ \\
\hline 10784. 36 & 10 & 3 & I & $\ldots$ & & & & & \\
\hline 10815. 24 & 2 & $\ldots$ & I & $\ldots \ldots$ & & & & & \\
\hline 10851. 65 & 6 & 1 & $\mathrm{I}$ & ..... & & & & & \\
\hline
\end{tabular}

Washington, June 23, 1958. 